\title{
The SWELL1-LRRC8 complex regulates endothelial AKT-eNOS-mTOR signaling and
}

3 Ahmad F. Alghanem ${ }^{1 \#}$, Chau Ta ${ }^{1}$, Joshua M. Maurer ${ }^{1}$, Susheel K. Gunasekar ${ }^{1}$, Ashutosh

4 Kumar $^{1}$, Urooj Fatima ${ }^{2}$, Chen $\mathrm{Kang}^{1}$, Litao Xie ${ }^{1}$, Oluwaseun Adeola ${ }^{2}$, Javier Abello ${ }^{3}$, Megan

5 Riker $^{4}$, Macaulay Elliot-Hudson ${ }^{2}$, Rachel A. Minerath ${ }^{2}$, Amber Stratman $^{3}$, Chad E. Grueter ${ }^{2}$,

6 Robert F. Mullins ${ }^{4}$ and Rajan Sah ${ }^{* 1,5}$

$7 \quad{ }^{1}$ Department of Internal Medicine, Cardiovascular Division, Washington University School of

8 Medicine, St. Louis, MO, USA.

$9{ }^{2}$ Department of Internal Medicine, Cardiovascular Division, University of lowa, lowa City, IA,

10 USA.

11 3epartment of Cell Biology and Physiology, Washington University in St. Louis, School of

12 Medicine, St. Louis, MO 63110

13

${ }^{4}$ Department of Ophthalmology, University of lowa, Carver College of Medicine, lowa City, IA,

15 USA.

${ }^{5}$ Center for Cardiovascular Research, Washington University, St Louis, MO, USA

${ }^{\#}$ Current address: King Abdullah International Medical Research Center (KAIMRC)-Eastern

${ }^{*}$ Correspondence:

Dr. Rajan Sah

Associate Professor of Medicine

425 S Euclid Ave

Washington University School of Medicine

BJICH 9606

St. Louis, MO 63110

Email: rajan.sah@wustl.edu 


\section{Abstract:}

32 The endothelium responds to a multitude of chemical and mechanical factors in regulating

33 vascular tone, angiogenesis, blood pressure and blood flow. The endothelial volume regulatory

34 anion channel (VRAC) has been proposed to be mechano-sensitive, to activate in response to

35 fluid flow/hydrostatic pressure and putatively regulate vascular reactivity and angiogenesis.

36 Here, we show that the Leucine Rich Repeat Containing Protein 8a, LRRC8a (SWELL1)

37 functionally encodes VRAC in human umbilical vein endothelial cells (HUVECs). Endothelial

38 SWELL1 (SWELL1) expression positively regulates AKT-eNOS signaling while negatively 39 regulating mTOR signaling, via a SWELL1-GRB2-Cav1-eNOS signaling complex. Endothelium-

40 restricted SWELL1 KO (SWELL1 KO) mice exhibit enhanced tube formation from ex-vivo aortic

41 ring explants in matrigel angiogenesis assays, develop hypertension in response to chronic 42 angiotensin II infusion and have impaired retinal blood flow with both diffuse and focal blood 43 vessel narrowing in the setting of Type 2 diabetes (T2D). These data demonstrate that SWELL1

44 antithetically regulates AKT-eNOS and mTOR signaling in endothelium and is required for 45 maintaining vascular function, particularly in the setting of T2D. 
Introduction

The endothelium integrates mechanical and chemical stimuli to regulate vascular tone, angiogenesis, blood flow and blood pressure(1). Endothelial cells express a variety of mechanosensitive ion channels that regulate vascular function(2), including TRPV4(3-6) and

52 Piezo1(7-9). The volume-regulated anion current (VRAC) is also prominent in endothelium, has been proposed to be mechano-sensitive(10), to activate in response to fluid flow/hydrostatic pressure(11) and putatively regulate vascular reactivity. However, the molecular identity of this endothelial ion channel has remained a mystery for nearly two decades.

SWELL1 or LRRC8a (Leucine-Rich Repeat Containing Protein 8a) encodes a transmembrane protein first described as the site of a balanced translocation in an immunodeficient child with agammaglobulinemia and absent B-cells $(12,13)$. Subsequent work revealed the mechanism for this condition to be due to impaired SWELL1-dependent GRB2-PI3K-AKT signaling in

61 lymphocytes, resulting in a developmental block in lymphocyte differentiation(14). Thus, for $\sim 11$

62 years, SWELL1 was conceived of as a membrane protein that regulates PI3K-AKT mediated

63 lymphocyte function(12,13). Although SWELL1 had been predicted to form a hetero-hexameric

64 ion channel complex with other LRRC8 family members(15), it was not until 2014 that

65 SWELL1/LRRC8a was shown to form an essential component of the volume-regulated anion

66 channel $(\operatorname{VRAC})(16,17)$, forming hetero-hexamers with LRRC8b-e $(17,18)$. Therefore,

67 historically, SWELL1-LRRC8 complex was first described as a membrane protein that signaled

68 via protein-protein interactions and then later found to form an ion channel signaling complex.

70 We showed previously that SWELL1 (LRRC8a) is an essential component of VRAC in 71 adipocytes that is required for insulin-PI3K-AKT2 signaling to mediate adipocyte hypertrophy 72 and systemic glucose homeostasis(19-21). The PI3K-AKT-eNOS signaling pathway is central to 
73 transducing both mechanical stretch(22) and hormonal inputs (insulin) to regulate endothelial

74 nitric oxide synthase (eNOS) expression and activity, which, in turn regulates vasodilation

75 (blood flow and pressure), inhibits leukocyte aggregration, and limits proliferation of vascular

76 smooth muscle cells (atherosclerosis). Indeed, insulin resistance is thought to be a systemic

77 disorder in the setting of Type 2 diabetes (T2D), affecting endothelium in addition to traditional

78 metabolically important tissues, such as adipose, liver, and skeletal muscle(23-25). In fact,

79 insulin resistant endothelium and the resultant impairment in PI3K-AKT-eNOS signaling has been proposed to underlie much of the endothelial dysfunction observed in the setting of obesity and T2D, predisposing to hypertension, atherosclerosis and vascular disease(23-25).

82

In this study, we demonstrate that VRAC is SWELL1-dependent in endothelium, associates with GRB2, caveolin-1 (Cav1), endothelial nitric oxide synthase (eNOS), and regulates PI3K-AKTeNOS, ERK1/2 and mTOR signaling - suggesting that SWELL1-LRRC8 channel complexes link insulin and mechano-signaling in endothelium. SWELL1-dependent AKT-eNOS, ERK1/2 and mTOR signaling influences angiogenesis, blood pressure and vascular function in vivo, while impaired endothelial SWELL1-LRRC8 signaling predisposes to vascular dysfunction in the setting of diet-induced T2D. 


\section{Results}

\section{SWELL1 functionally encodes VRAC in endothelium}

93 The volume-regulatory anion current (VRAC) has been measured and characterized in

94 endothelial cells for decades but the molecular identity of this endothelial ion channel remains

95 elusive $(10,11,26)$. To determine if the leucine-rich repeat containing membrane protein

96 SWELL1 (LRRC8a) recently identified in cell lines $(16,17)$ is required for VRAC in endothelial

97 cells, as it is in adipocytes (19), pancreatic $\beta$-cells $(27,28)$, nodose neurons (29) and spermatozoa (30), we first confirmed robust SWELL1 protein expression by Western blot (Figure 1A) and immunostaining (Figure 1B) in human umbilical vein endothelial cells (HUVECs). SWELL1 protein expression is substantially reduced upon adenoviral transduction

101 with a short-hairpin RNA directed to SWELL1 (Ad-shSWELL1-mCherry) as compared to a 102 scrambled control (Ad-shSCR-mCherry). Next, we measured hypotonically-induced (210

103 mOsm) endothelial VRAC currents in HUVECs. These classic outwardly rectifying hypotonically-

104 induced VRAC currents are prominent in HUVECs, largely blocked by the VRAC inhibitor 4-(2-

105 Butyl-6,7-dichloro-2-cyclopentyl-indan-1-on-5-yl) oxobutyric acid (DCPIB; Figure 1C\&D), and

106 significantly suppressed upon shSWELL1-mediated SWELL1 knock-down (Figure 1E\&F),

107 consistent with SWELL1 functionally encoding endothelial VRAC. 
A

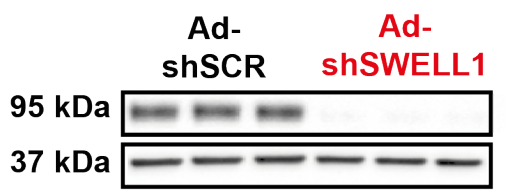

IB:SWELL1 IB:GAPDH
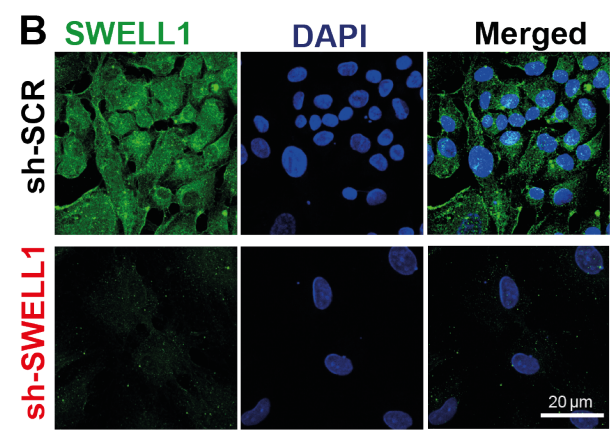

C
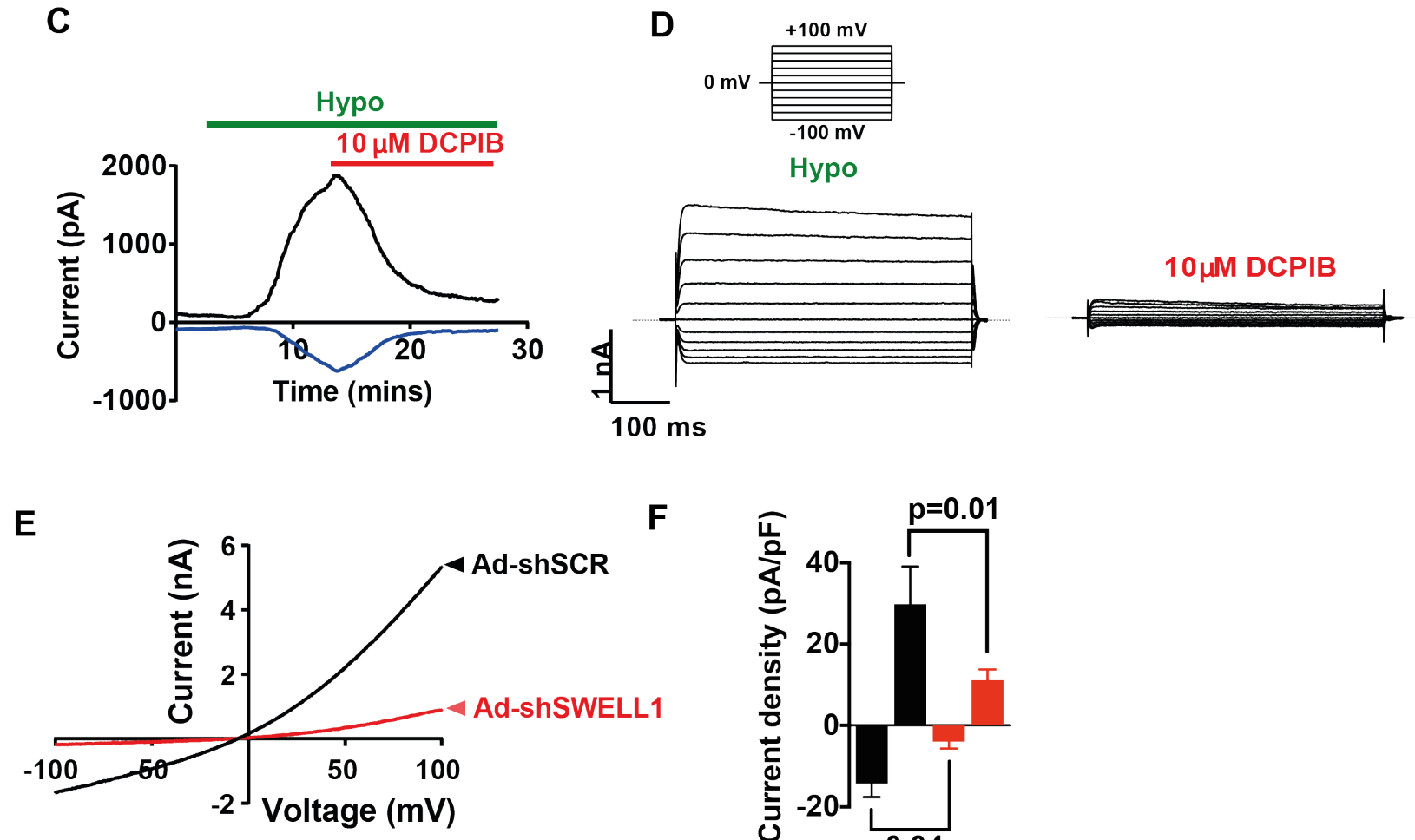

Figure 1. SWELL1 mediates VRAC currents in human umbilical vein endothelial cells (HUVECs).

A, SWELL1 western blot in HUVECs transduced with adenovirus expressing a short hairpin RNA directed to SWELL1 (Ad-shSWELL1) compared to control scrambled short hairpin RNA (Ad-shSCR). GAPDH is used as loading control. B, Immunofluorescence staining of the HUVECs transduced with AdshSWELL1 and Ad-shSCR. C, Current-time relationship of VRAC (hypotonic, $210 \mathrm{mOsm}$ ) in Ad-shSCR transduced HUVEC and co-application of $10 \mu \mathrm{M}$ DCPIB. D, Representative current traces upon hypotonic activation (left) during voltage steps (from -100 to $+100 \mathrm{mV}$, shown in inset) and inhibition by DCPIB (right). E, Current-voltage relationship of VRAC during voltage ramps from $-100 \mathrm{mV}$ to $+100 \mathrm{mV}$ after hypotonic swelling in HUVECs transduced with Ad-shSCR and Ad-shSWELL1. F. Mean current outward and inward densities at +100 and $-100 \mathrm{mV}$ ( $\mathrm{n}$,sh-SCR $=4$ cells; $\mathrm{n}$,shSwELL1 $=6$ cells). Data are shown as mean \pm s.e.m. ${ }^{*} p<0.05 ;{ }^{* *} p<0.01$; unpaired t-test for $\mathbf{F}$. 


\section{SWELL1 regulates PI3K-AKT-eNOS, ERK and mTOR signaling in endothelium}

122 Previous studies in adipocytes demonstrate that SWELL1 regulates insulin-PI3K-AKT signaling,

123 adipocyte expansion and systemic glycemia, whereby SWELL1 loss-of-function induces an

124 insulin-resistant pre-diabetic state $(19,20)$. Insulin signaling is also important in regulating

125 endothelium and vascular function $(24,25,31)$. Moreover, insulin-resistance in Type 2 diabetes

126 (T2D) is considered a systemic disorder and insulin-resistant endothelium is postulated to

127 underlie impaired vascular function in T2D $(24,25)$. As SWELL1 is highly expressed in

128 endothelium (Figure 1), and PI3K-AKT-eNOS signaling critical for endothelium-dependent

129 vascular function(32), we next examined AKT-eNOS, ERK1/2 and mTOR signaling in SWELL1

130 KD compared to control HUVECs under basal conditions. Basal phosphorylated AKT2 (pAKT2,

131 Figure 2A\&D), pAKT1 (Figure 2A\&E), p-eNOS (Figure 2A\&C), pERK1/2 (Figure 2A\&F) are

132 abrogated in HUVECs upon SWELL1 KD, indicating that SWELL1 contributes to AKT-eNOS,

133 and ERK signaling in endothelium. Curiously, basal pS6 ribosomal protein, indicative of mTOR

134 signaling, is augmented in SWELL1 KD HUVECs compared to control (Figure 2A\&G),

135 suggesting SWELL1 to be a negative regulator of mTOR in endothelium. As a complementary

136 approach, we used siRNA mediated SWELL1 knock-down using a silencer select siRNA

137 targeting SWELL1 mRNA with a different sequence from shSWELL1. siRNA mediated SWELL1

138 KD in HUVECs yielded nearly identical results to the shRNA KD approach (Figure 2-Figure

139 Supplement 1). In summary, SWELL1 expression level regulates AKT, eNOS, ERK and mTOR 140 signaling in endothelium. 
A

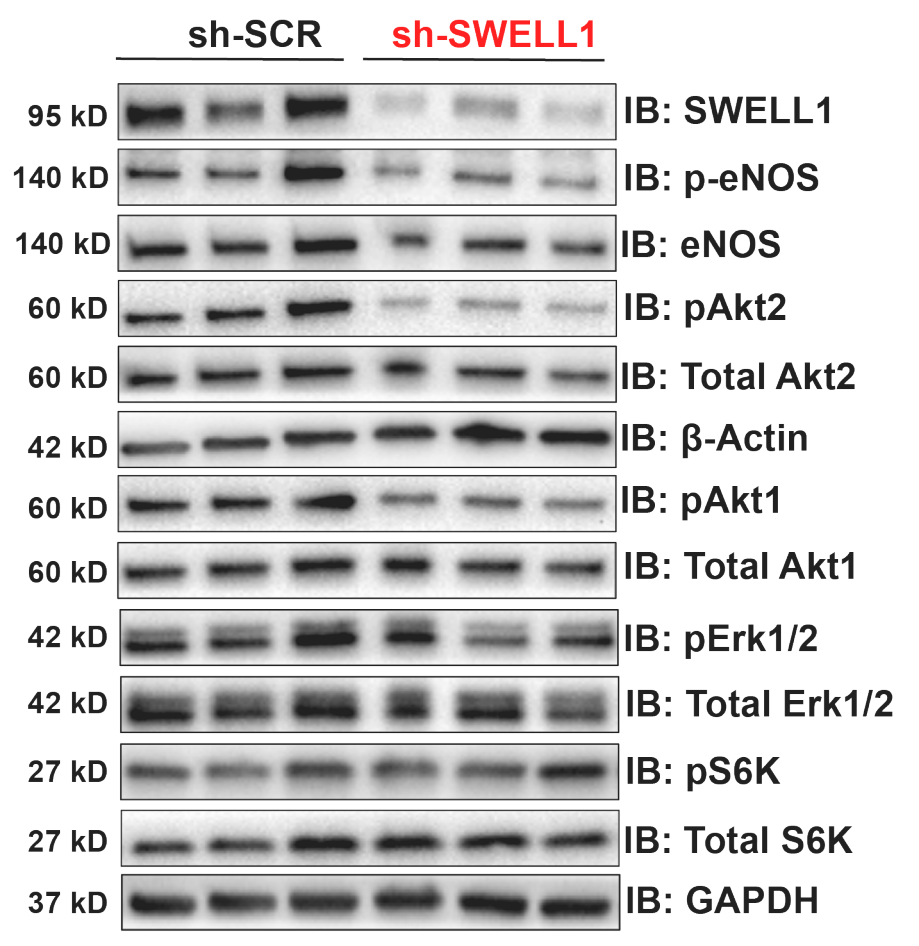

B SWELL1

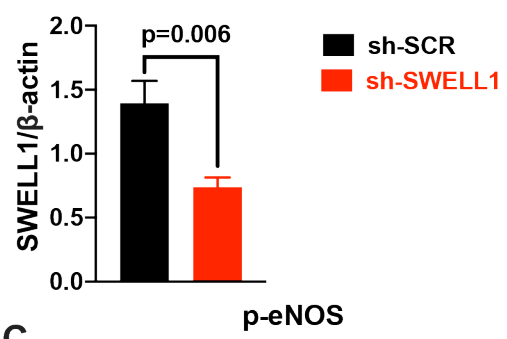

C
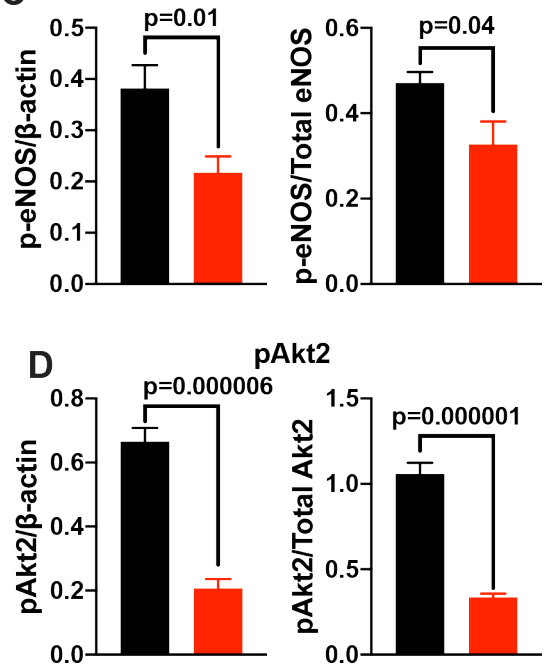

E
F

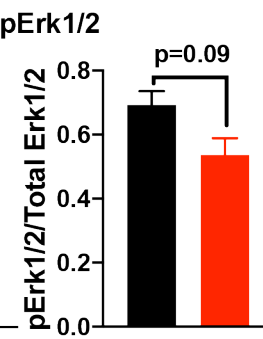

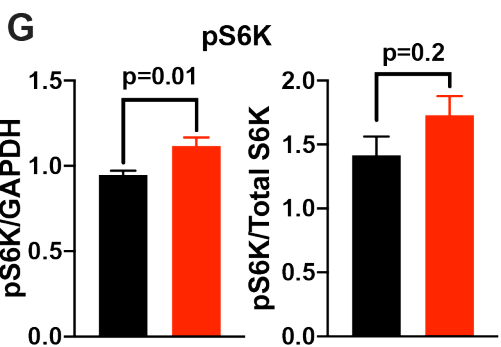

\section{Figure 2. SWELL1 regulates PI3K-AKT-eNOS, ERK and mTOR signaling in endothelium.}

(A), Western blots of SWELL1, pAkt2, pAkt1, Akt2, Akt1, pErk1/2, Erk1/2, p-eNOS, eNOS, pS6K ribosomal protein, S6K ribosomal protein, GAPDH, and $\beta$-Actin in Ad-shSCR and Ad-shSWELL1 transduced HUVECS under basal conditions. Quantification of SWELL1/ $\beta$-Actin (B), p-eNOS/ $\beta$-actin, $p$ eNOS/Total eNOS (C), pAkt2/ $\beta$-actin, pAkt2/Total Akt2 (D), pAkt1/GAPDH, pAkt1/Total Akt1 (E), pERK1/2 /GAPDH, pErk1/2 /Total Erk1/2 (F), pS6 ribosomal protein/GAPDH, and pS6K ribosomal protein/Total S6K ribosomal protein $(\mathbf{G})$. $\mathrm{N}=6$ independent experiments. Significance between the indicated groups in all blots were calculated using a two-tailed Student's t-test. P-values are illustrated on figures. Error bars represent mean \pm s.e.m. 
A

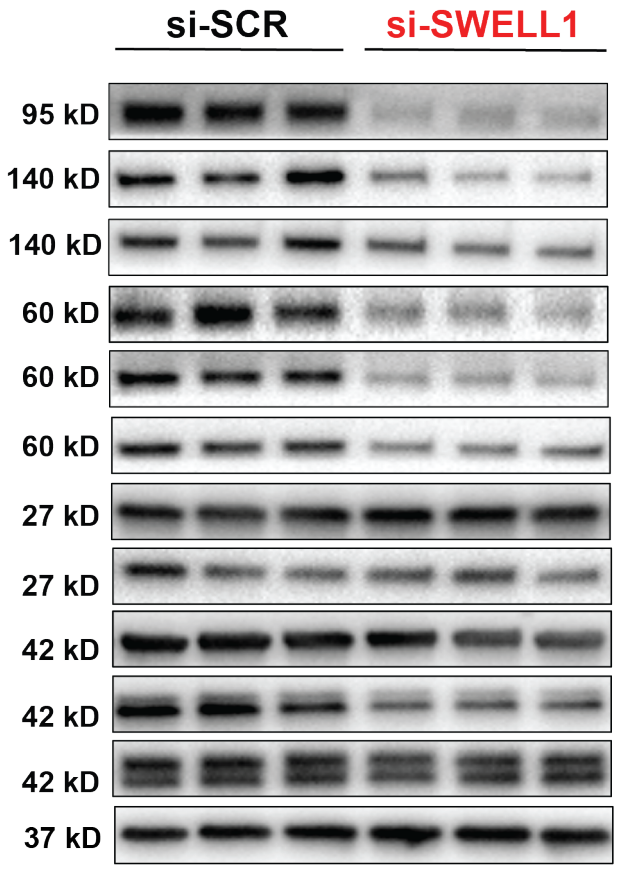

IB: SWELL1

IB: $p$-eNOS

IB: eNOS

IB: pAkt2

IB: pAkt1

IB: Akt

IB: pS6K

IB: Total S6K

IB: $\beta$-Actin

IB: pErk1/2

IB: Total Erk1/2

IB: GAPDH

pErk1/2
B
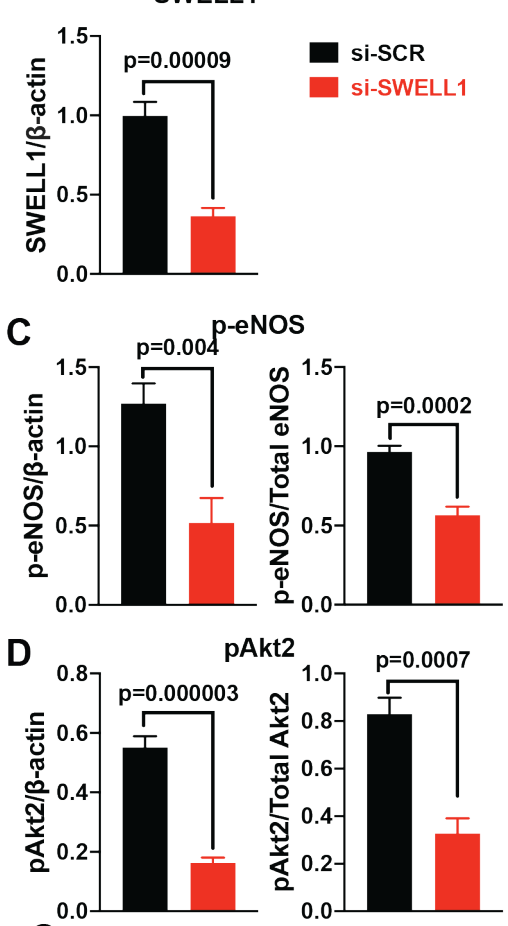

$\mathbf{G}$

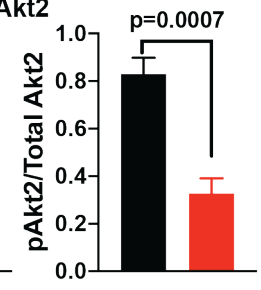

pS6K

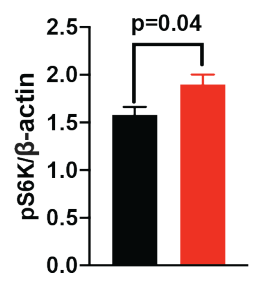

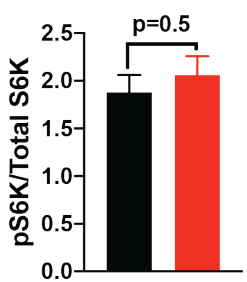
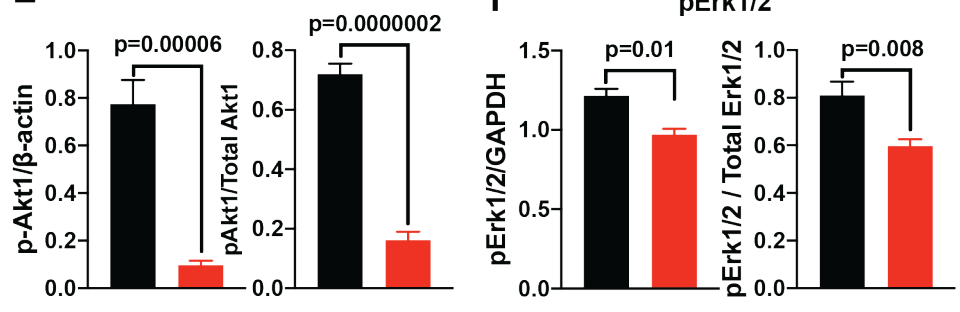

Figure 2- Figure Supplement 1. SWELL1 regulates PI3K-AKT-eNOS, ERK and mTOR signaling in endothelium.

(A), Western blots of SWELL1, pAkt2, pAkt1, Akt, pErk1/2, Erk1/2, p-eNOS, eNOS, pS6K ribosomal protein, S6K ribosomal protein, and $\beta$-Actin in si-SCR and si-SWELL1 transduced HUVECS under basal conditions. Quantification of SWELL1/ $\beta$-Actin (B), p-eNOS/ $\beta$-actin, p-eNOS/Total eNOS (C), pAkt2/ $\beta$ actin, pAkt2/Total Akt (D), pAkt1/ $\beta$-actin, pAkt1/Total Akt (E), pERK1/2 / $\beta$-actin, pErk1/2 /Total Erk1/2 $(\mathbf{F})$, pS6 ribosomal protein/ $\beta$-actin, and pS6K ribosomal protein/Total S6K ribosomal protein $(\mathbf{G}) . \mathrm{N}=6$ independent experiments. Significance between the indicated groups in all blots were calculated using a two-tailed Student's t-test. P-values are illustrated on figures. Error bars represent mean \pm s.e.m.

SWELL1 interacts with GRB2, Cav1 and eNOS and mediates stretch-dependent eNOS

\section{3 signaling}

164 In adipocytes, the mechanism of SWELL1-mediated regulation of PI3K-Akt signaling involves

165 SWELL1/GRB2/Cav1 molecular interactions (19). To determine if SWELL1 resides in a similar 
166 macromolecular signaling complex in endothelium we immunoprecipitated (IP) endogenous

167 GRB2 from HUVECs. Upon GRB2 IP, we detect SWELL1 protein in shSCR treated HUVECs

168 and less SWELL1 upon GRB2 IP from shSWELL1-treated HUVECs, consistent with a SWELL1-

169 GRB2 interaction (Figure 3A\&B). In addition, with GRB2 IP we also detect both Cav1 (Figure

1703 A\&B) and eNOS (Figure 3B). These data suggest that endothelial SWELL1 resides in a

171 signaling complex that includes GRB2, Cav1 and eNOS, consistent with the findings that GRB2

172 and Cav1 interact, and that Cav1 regulates eNOS via a direct interaction (33-35). Also, GRB2

173 has been shown to regulate endothelial ERK, AKT and JNK signaling (36). Moreover, these

174 data are also in-line with the notion that caveoli form mechanosensitive microdomains (37-39)

175 that regulate $\operatorname{VRAC}(40,41)$ and that VRAC can be activated by mechanical stimuli in a number

176 of cell types, including endothelium $(10,26,42-45)$. 
A

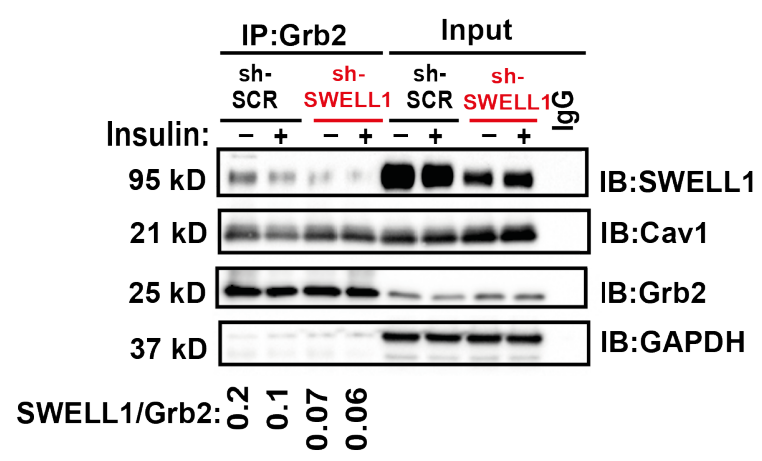

B

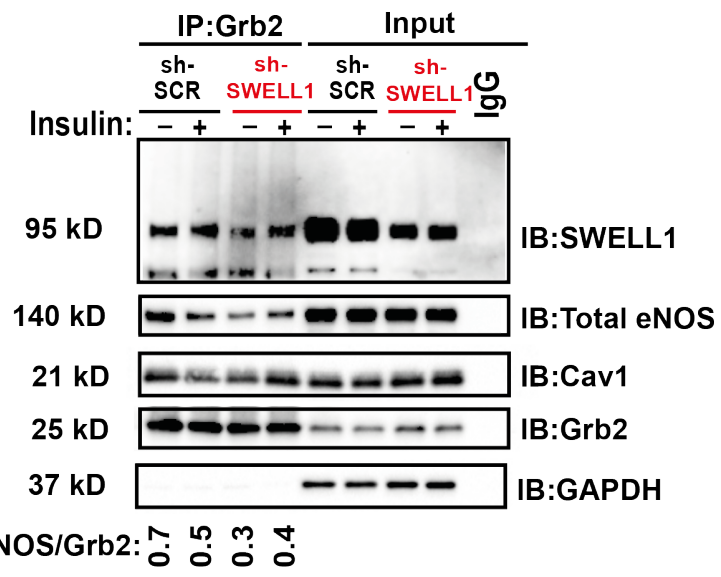

C

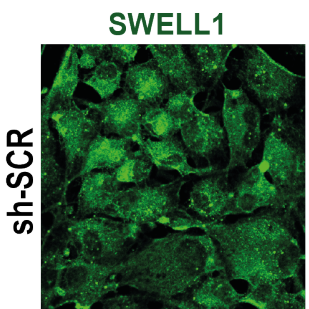
eNOS DAPI Merged
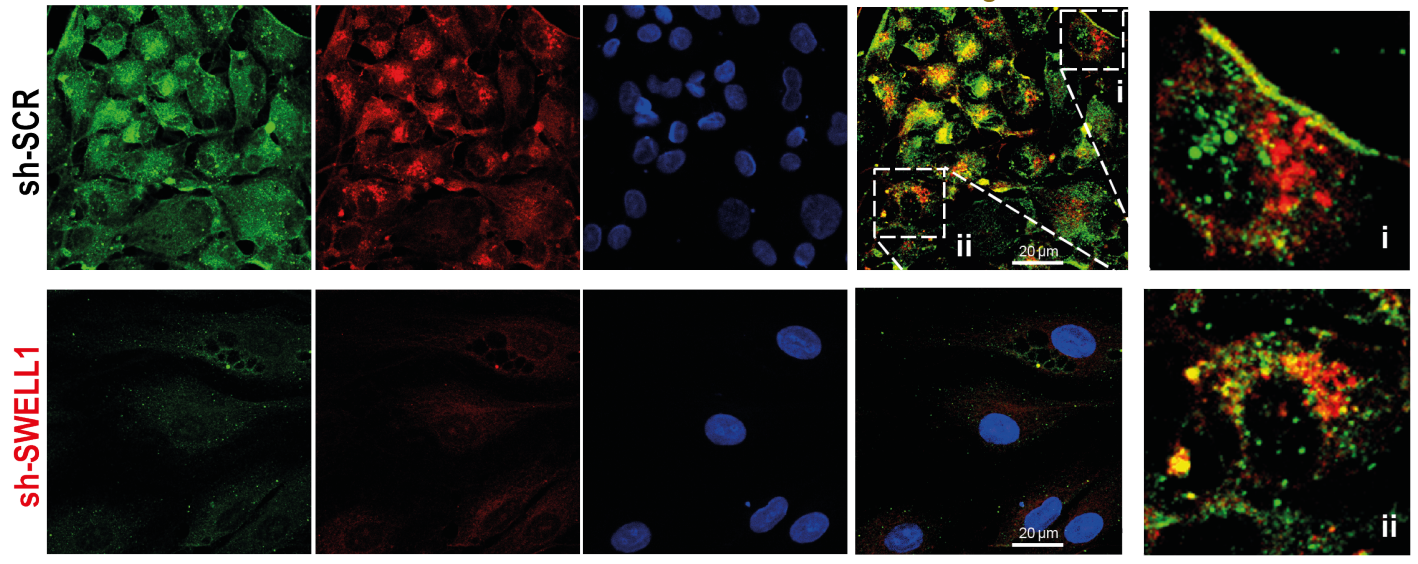

D

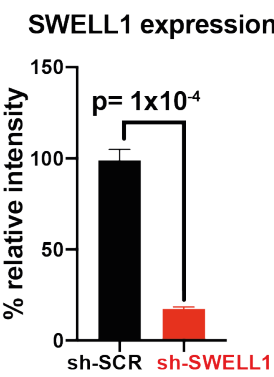

$E$

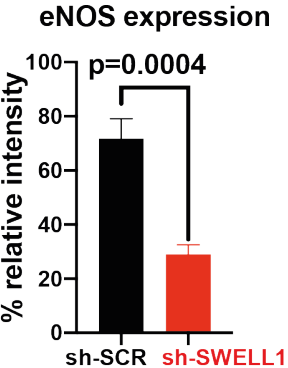

178 Figure 3. SWELL1 interacts with Grb2, Cav1 and eNOS in human endothelium

A, GRB2 immunoprecipitation from Ad-shSCR and Ad-shSWELL1 transduced HUVECs and immunoblot with SWELL1, Cav1 and GRB2 antibodies. Densitometry values for GRB2 co-immunoprecipitated SWELL1 (SWELL1/GRB2) and GRB2 co-immunoprecipitated Cav1 (Cav1/GRB2). GAPDH serves as loading control for input samples. (B) GRB2 immunoprecipitation from Ad-shSCR and Ad-shSWELL1 transduced HUVECs and immunoblot with SWELL1, eNOS, Cav1 and GRB2 antibodies. Insulinstimulation with $100 \mathrm{nM}$ insulin for 10 minutes. Densitometry values for GRB2 co-immunoprecipitated eNOS (eNOS/GRB2). Representative blots from 3 independent experiments. (C) Representative endogenous SWELL1 and eNOS immunofluorescence staining in Ad-shSCR and Ad-shSWELL1 
transduced HUVECs. Representative image from 6 independent experiments. (D-E) Quantification of SWELL1 ( $D, n=6)$ and eNOS $(E, n=6)$ immunofluorescence staining upon SWELL1 KD. Evidence of SWELL1-eNOS colocalization (C, insets) in plasma membrane (i) and perinuclear regions (ii).

190 Significance between the indicated groups are calculated using a two-tailed Student's t-test. P-values are 191 indicated on figures. Error bars represent mean \pm s.e.m.

193 We also examined the relationship between SWELL1 and eNOS protein expression and

194 localization in HUVECs by immunofluorescence (IF) staining (Figure 3C). Similar to observed

195 by Western blot (Figure 2A, and Figure 2-Figure Supplement 1), IF staining reveals that

196 reductions in SWELL1 expression correlate with reduced eNOS expression (Figure 3C-E,

197 Figure 3-Figure Supplement 1). Moreover, SWELL1 and eNOS co-localize in plasma

198 membrane and peri-nuclear intracellular domains (Figure 3C, inset), consistent with the IP data

199 revealing a SWELL1-GRB2-Cav-eNOS interaction (Figure 3A\&B), and also with previously

200 described intracellular eNOS localization(46). 
sh-SCR

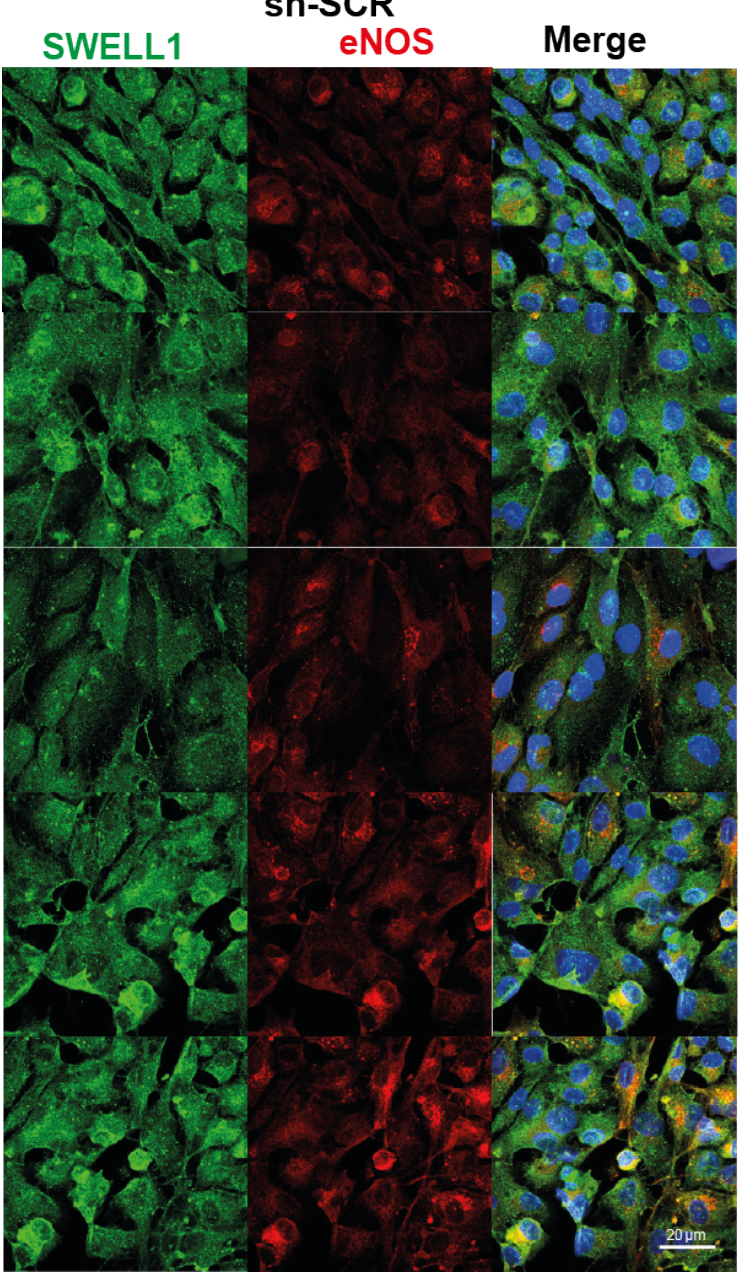

Sh-SWELL1

SWELL1 eNOS

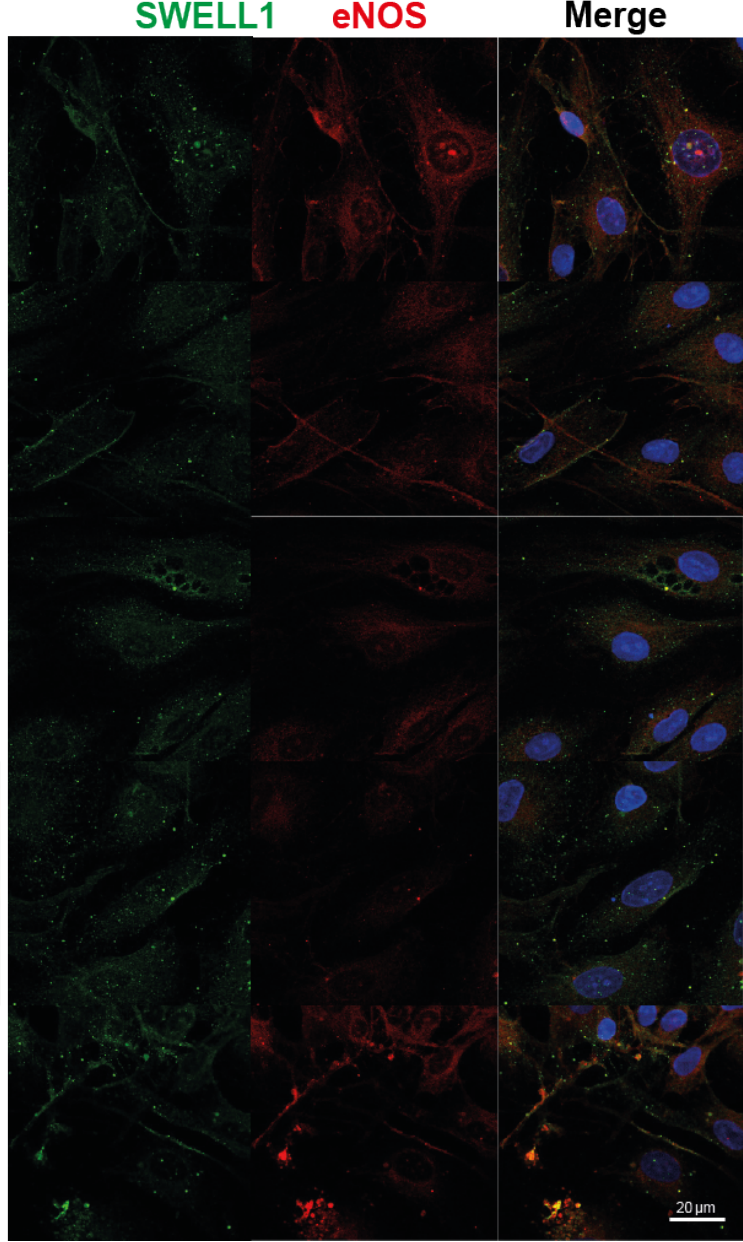

Figure 3 - Figure Supplement 1. SWELL1 co-localizes with eNOS and regulates eNOS expression

SWELL1 (green) and eNOS (red) immunofluorescence staining of HUVEC transduced with either AdshSCR or Ad-shSWELL1. Scale bar is $20 \mu \mathrm{m}$. DAPI (blue) labels nuclei.

Given that endothelial cells respond to stretch stimuli to regulate vascular tone via activation of eNOS, we next examined the SWELL1-dependence of stretch-induced AKT, ERK1/2 and eNOS signaling in HUVECs (Figure 4). Stretch (5\%) is sufficient to stimulate AKT1 and AKT2 signaling (Figure 4A-C), though not ERK1/2 signaling (Figure 4D) in HUVECs, and all are

210 blunted in SWELL1 KD HUVECS (Figure 4A-D). Similarly, we observe abrogation of time-

211 dependent p-eNOS signaling with 5\% stretch in SWELL1 KD HUVECS compared to control

212 (Figure 4E\&F). Taken together, these data position SWELL1 as a regulator stretch-mediated

213 PI3K-AKT-eNOS signaling in endothelium via a SWELL1-GRB2-Cav1-eNOS signaling complex. 

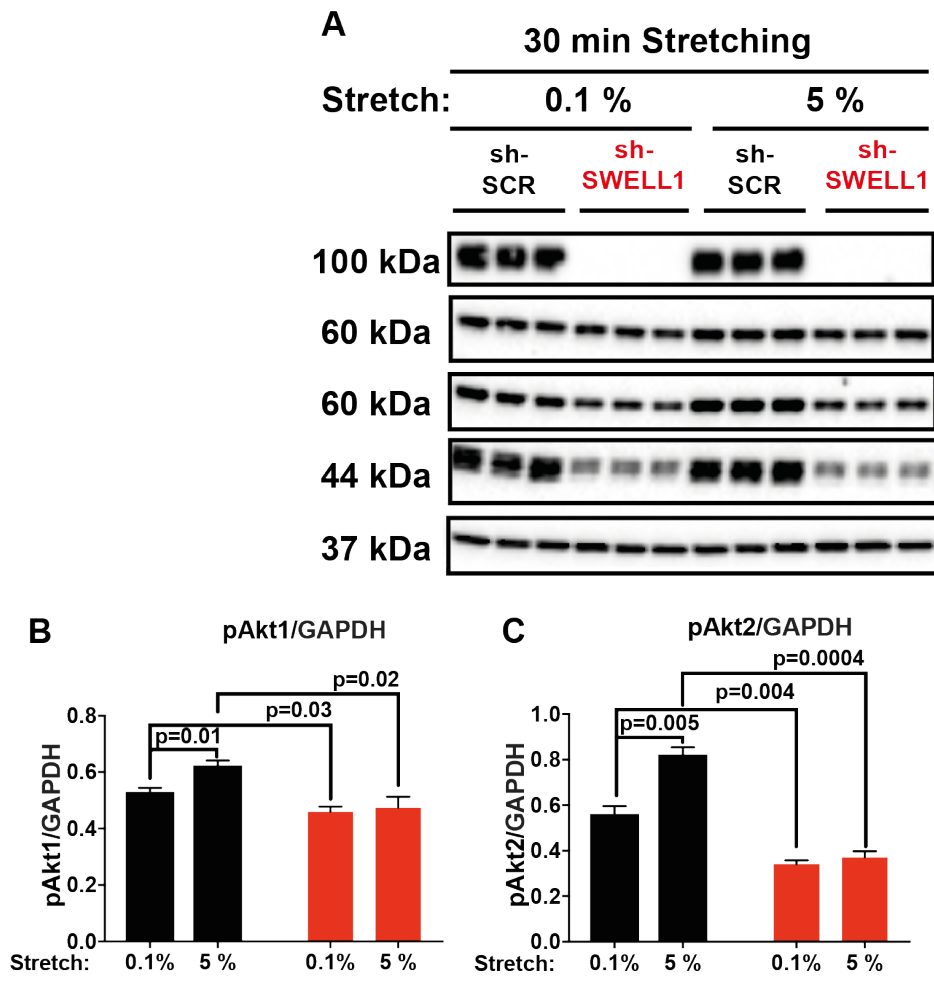

\section{IB:SWELL1}

IB:pAkt1

IB:pAkt2

IB:pErk1/2

IB:GAPDH
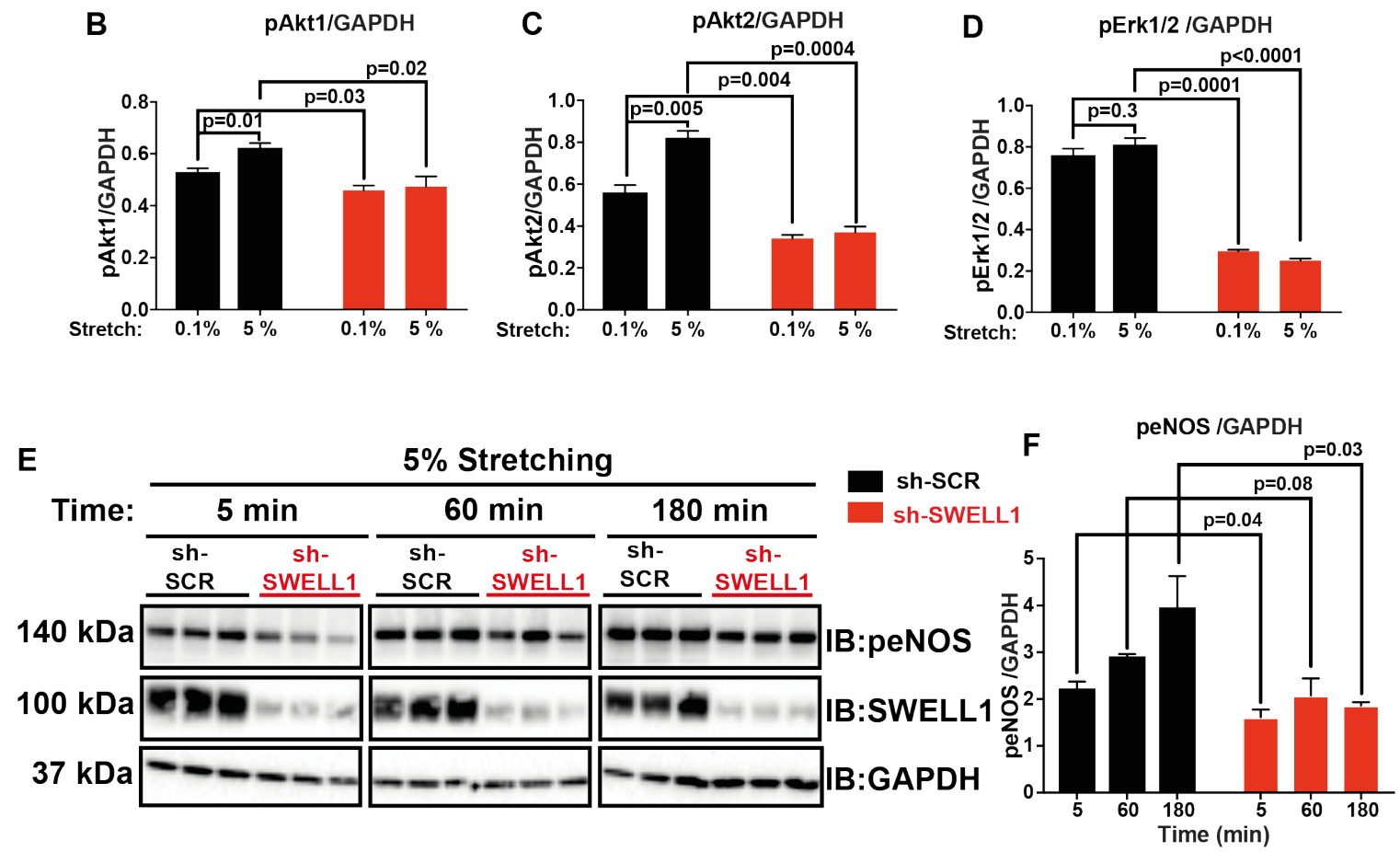

215 Figure 4. SWELL1 is required for intact stretch-induced AKT-eNOS signaling

A, Western blot of SWELL1, pAKT1, pAKT2, pERK1/2 in response to 30 minutes of $0.1 \%$ and $5 \%$ static stretch in Ad-shSCR and Ad-shSWELL1 transduced HUVECs. GAPDH is used as a loading control. (BD) Densitometry quantification from A of pAKT1 (B), pAKT2 (C) and pErk1/2 (D). (E) Western blot of peNOS, SWELL1, in response to $5 \%$ static stretching for 5, 60 and $180 \mathrm{~min}$ in Ad-shSCR and AdshSWELL1 transduced HUVECs. (F) Densitometry quantification from E of eNOS. GAPDH is used as a loading control. Significance between the indicated groups in all blots were calculated using a two-tailed Student's t-test. P-values are illustrated on figures. Error bars represent mean \pm s.e.m.

224 To examine the functional consequences of endothelial SWELL1 ablation in vivo we generated

225 endothelial-targeted SWELL1 KO mice (eSWELL1 KO) by crossing SWELL1 floxed mice (19,

226 27) with the endothelium-restricted CDH5-Cre mouse (CDH5-Cre;SWELL1 ${ }^{\text {fl/fl; }}$ Figure 5A). 
227 Patch-clamp recordings of primary endothelial cells isolated from WT and eSWELL1 KO mice

228 (Figure 5B) revealed robust hypotonically-activated currents (Hypo, $210 \mathrm{mOsm}$ ) in WT

229 endothelial cells, that are DCPIB inhibited (Figure 5C\&E), while eSWELL1 KO endothelial cells

230 exhibit markedly reduced hypotonically-activated currents (Figure 5D\&E). Immunofluorescence

231 staining of aortic ring explants revealed that SWELL1 ablation from CD31+ primary endothelial

232 cells significantly enhanced ex-vivo sprouting angiogenesis from these explants (Figure 5F\&G),

233 based on both tube length and number of tip cells in eSWELL1 KO mice as compared to WT

234 mice (Figure 5\&H-I), suggesting that SWELL1 regulates angiogenesis. Indeed, genome-wide

235 transcriptome analysis of SWELL1 KD HUVEC compared to control (RNA sequencing) reveal

236 multiple pathways enriched regulating angiogenesis, migration and tumorigenesis, including

237 GADD45, IL-8, p70S6K (mTOR), TREM1, angiopoeitin and HGF signaling (Figure 6,

238 Supplementary Table 1\&2). Also, notable are statistically significant increases in VEGFA (1.6-

239 fold) and CD31 (2.0-fold) expression in SWELL1 KD HUVECs, both of which are pro-angiogenic

240 and associated with mTORC1 hyperactivation(47). Pathways linked to cell adhesion and renin-

241 angiotensin signaling are also enriched - both pathways and processes that are known to be

242 altered in vasculature in the setting of atherosclerosis and Type 2 diabetes (T2D). Finally, the

243 trends toward reduced eNOS protein expression observed upon SWELL1 KD in HUVECs

244 (Figure 2A, Supplementary Fig 1A, Fig. 3C, Figure 3-Figure Supplement 1) are associated

245 with reduced eNOS mRNA expression (0.54-fold in SWELL1 KD HUVEC, $p<10^{-4}$ ). 
A

A B
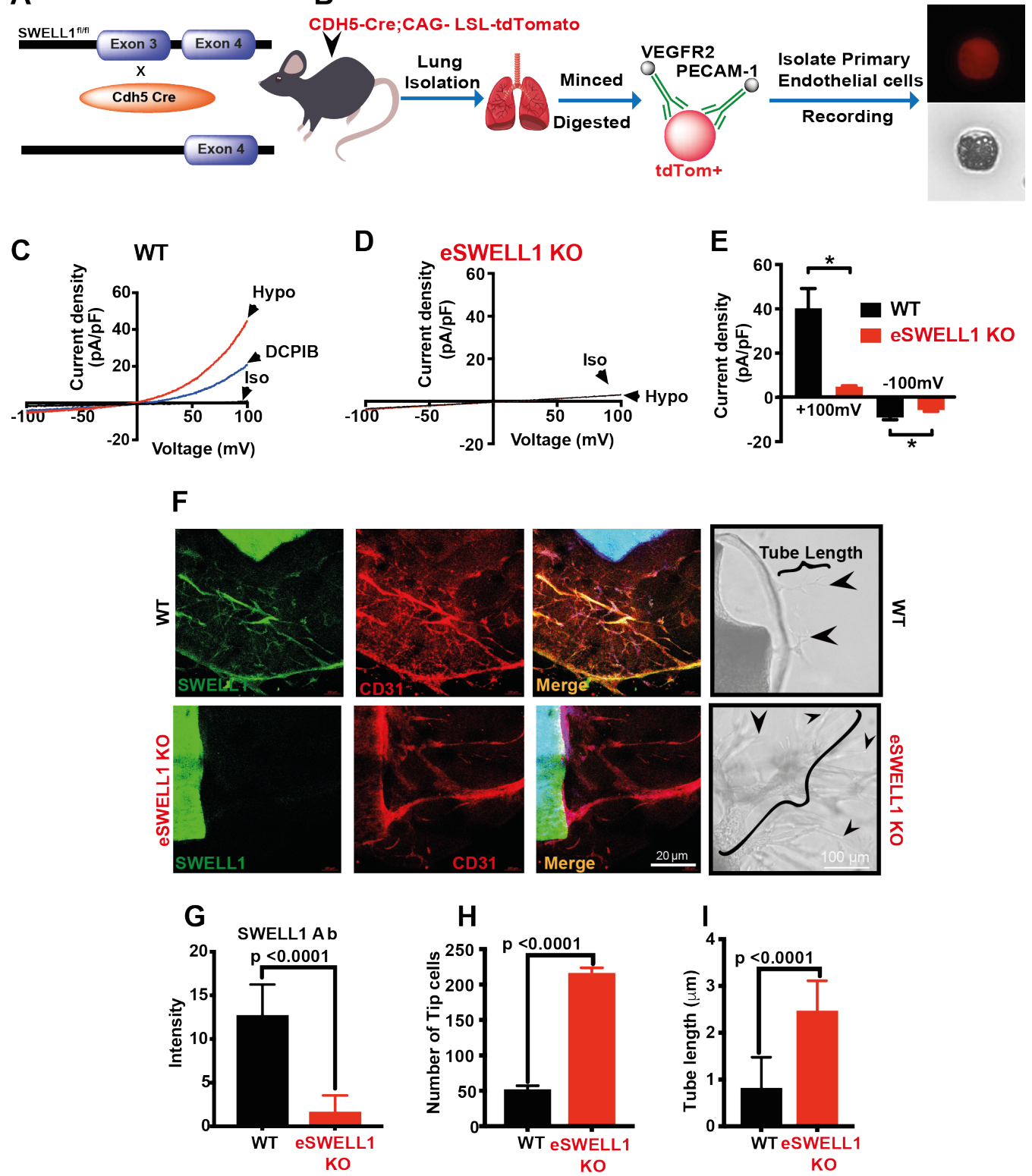

Figure 5. Endothelium-specific SWELL1 KO mice exhibit enhanced tube formation from aortic explants

A, Strategy for endothelium targeted SWELL1 ablation to generate eSWELL1 KO. (B) Isolation of murine primary endothelial cells from WT and eSWELL1 KO using tdTomato reporter mice. C-D, Current-voltage relationships of VRAC in isotonic (Iso, $300 \mathrm{mOsM}$ ) and hypotonic (Hypo, $210 \mathrm{mOsm}$ ) solution in response to voltage ramps from -100 to $+100 \mathrm{mV}$ over $500 \mathrm{~ms}$ in WT (C) and $\mathrm{KO}$ (D) primary murine endothelial cells. DCPIB $(10 \mu \mathrm{M})$ inhibition in C (WT). (E) Mean outward $(+100 \mathrm{mV})$ and inward $(-100 \mathrm{mV})$ currents from WT ( $n=3$ cells) and eSWELL1 KO ( $n=3$ cells). (F) Ex-vivo aorta sprouting assay performed in aortic rings isolated from WT and eSWELL1 KO mice and cultured in FGM media for 3 days at 37oC. Immunofluoresence staining with antibodies to SWELL1 (green), CD31 (red), SWELL1+CD31+ (Merge) and bright field images show endothelial cell tubes sprouting from WT and eSWELL1 KO aortic rings (black arrow heads). (G-I) Quantification of SWELL1 immunofluorescence signal (G, WT $=15, K O=15$ ), number of tip cells $(\mathbf{H}, \mathrm{WT}=26, \mathrm{KO}=31)$, and endothelial tube length $(\mathbf{I}, \mathrm{WT}=30, \mathrm{KO}=30)$ in WT and eSWELL1 KO aortic explants. Statistical significance between the indicated values calculated using a 
bioRxiv preprint doi: https://doi.org/10.1101/2020.08.04.236182; this version posted Auqust 4, 2020. The copyright holder for this preprint (which was not certified by peer review) is the author/funder, who has granted bioRxiv a license to display the preprint in perpetuity. It is made available under aCC-BY 4.0 International license.

two-tailed Student's t-test. P-values are illustrated on figures. Error bars represent mean \pm s.e.m. $n=3$, independent experiments.

\section{A}

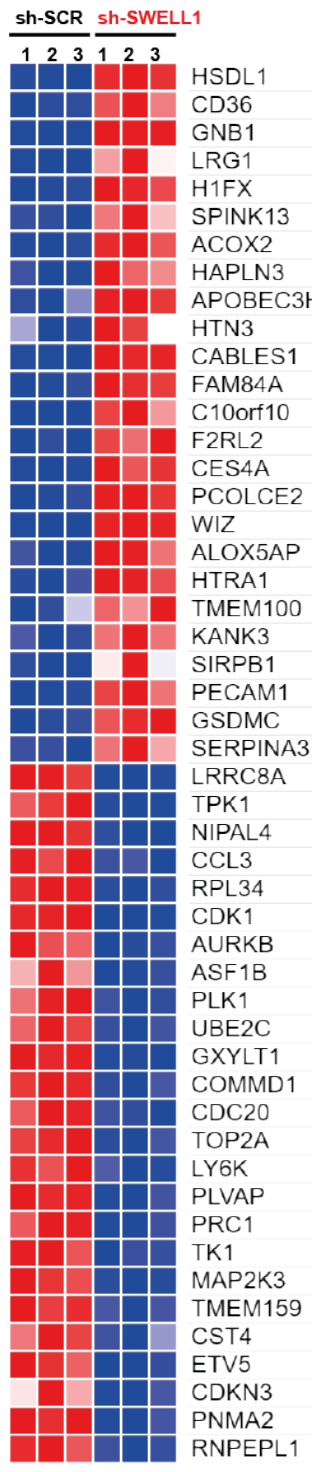

B

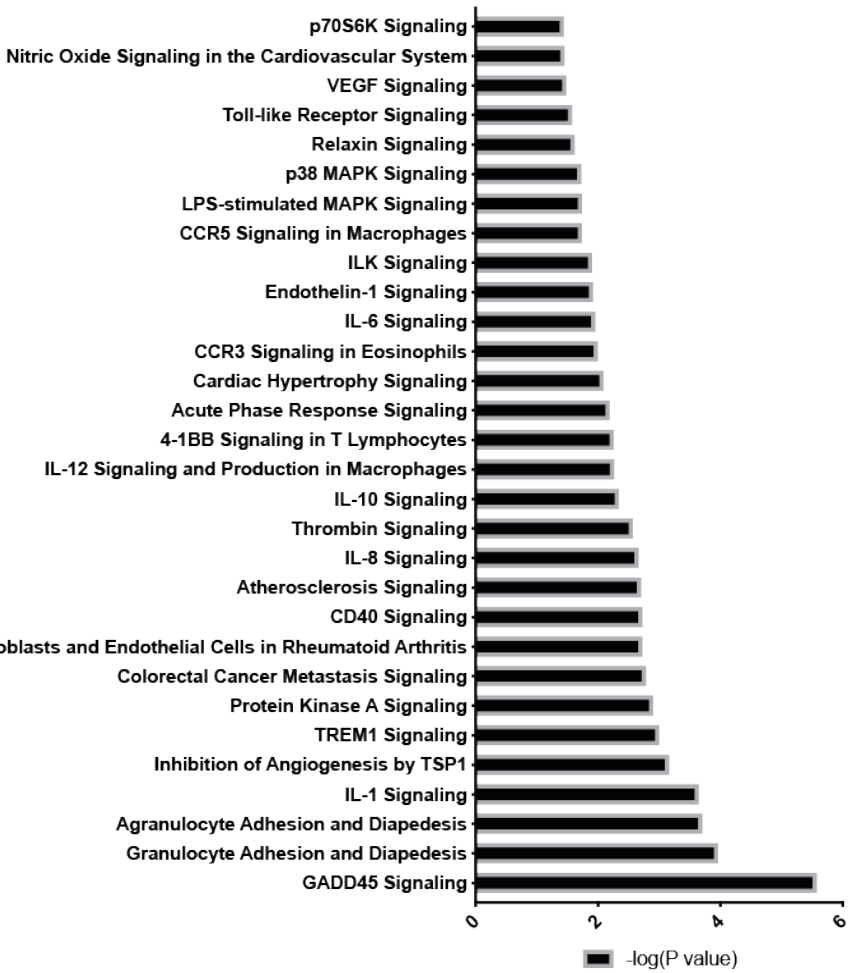

Role of Macrophages, Fibroblasts and Endothelial Cells in Rheumatoid Arthritis Colorectal Cancer Metastasis Signaling

IL-1 Signaling and Diapedesis

265 Figure 6. RNA sequencing of Ad-shSCR and Ad-shSWELL1 transduced HUVECs.

A, Heatmap analysis displaying top 25 upregulated or 25 downregulated genes between shSCR and shSWELL1. (B) IPA canonical pathway analysis of genes significantly regulated by shSWELL1 in comparison to shSCR. $\mathrm{n}=3$ for each group. For analysis with IPA, FPKM cutoffs of 1.5, fold change of $\geq 1.5$, and false discovery rate $<0.05$ were utilized for significantly differentially regulated genes. 
eSWELL1 KO mice exhibit mild angiotensin-II stimulated hypertension and impaired

273 retinal blood flow in the setting of Type 2 diabetes

274 Based on our findings that SWELL1 regulates AKT-eNOS signaling in endothelium, and that

275 eNOS signaling is central to blood pressure regulation, we next examined blood pressures in

276 eSWELL1 KO mice compared to WT controls (SWELL1//fli mice). Male mice exhibit no

277 significant differences in systolic blood pressure under basal conditions (Figure 7A), while

278 female mice are mildly hypertensive relative to WT mice (Figure 7B). However, after 4 weeks of

279 angiotensin-II infusion (Ang II), male eSWELL1 KO mice develop exacerbated systolic

280 hypertension as compared to Angll-treated WT mice (Figure 7C). These data are consistent

281 with endothelial dysfunction and impaired vascular relaxation in eSWELL1 KO mice, resulting in

282 a propensity for systolic hypertension.

A

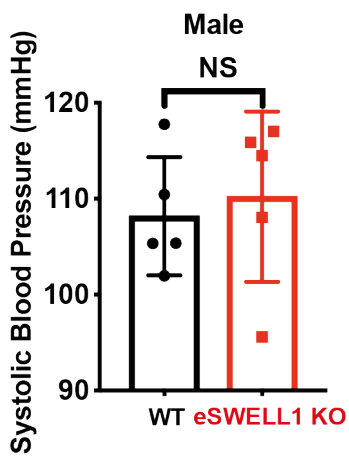

B

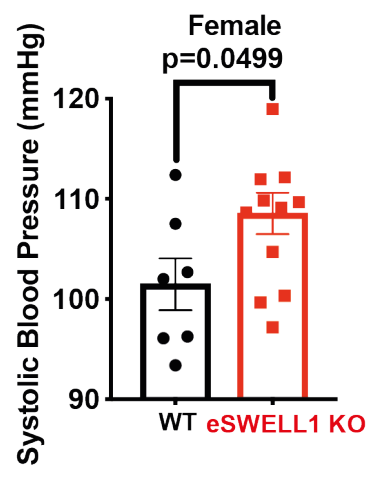

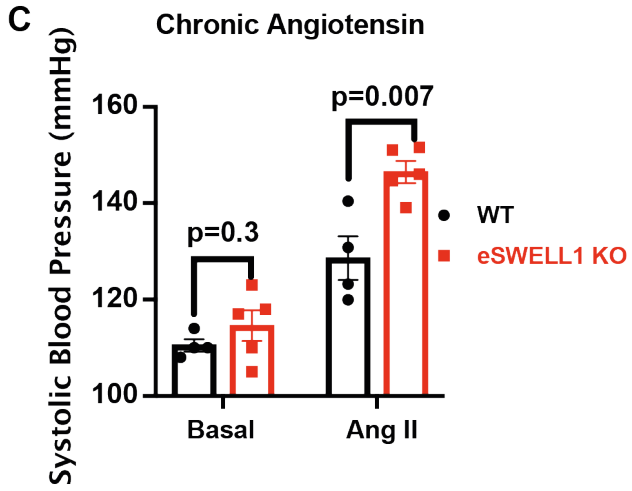

Figure 7. Endothelial-targeted SWELL1 deletion predisposes to systolic hypertension

Tail-cuff systolic blood pressures of (A) male and (B) female WT ( $n=5$ males and 7 females) and eSWELL1 KO ( $n=5$ males and 12 females) mice. (C) Systolic blood pressures of male WT $(n=4)$ and eSWELL1 KO $(n=5)$ mice under basal conditions and after 4 weeks of chronic angiotensin II infusion. Statistical significance between the indicated values calculated using a two-tailed Student's t-test. Pvalues are illustrated on figures. Data are shown as mean \pm s.e.m.

As endothelial dysfunction may also result in impaired blood flow we performed retinal imaging during i.p. injection of fluorescein to assess retinal vessel blood flow and morphology in WT and eSWELL1 KO mice. Mice raised on a regular diet have mild, non-significant impairments in retinal blood flow, based on the relative rate of rise of the fluorescein signal in retinal vessels 
295 (Figure 8-Figure Supplement 1). There is also evidence of mild focal narrowing of retinal

296 vessels in eSWELL1 KO as compared to WT mice (Figure 8-Figure Supplement 1A, D\&E),

297 with no significant differences in other parameters (Figure 8-Figure Supplement 1G-L). In

298 mice raised on high-fat high-sucrose (HFHS) diet, retinal blood flow is more severely impaired

299 (Figure 8A-C) with significant focal, and diffuse retinal vessel narrowing in eSWELL1 KO mice

300 compared to WT mice (Figure 8A; F-H, Figure 8-Figure Supplement 2, Figure 8-Video 1),

301 and this relative difference is markedly worse in female compared to male mice. These findings

302 are all consistent with endothelial dysfunction and impaired retinal vessel vasorelaxation due to

303 reduced eNOS expression and activity, particularly in the setting of HFHS diet. Also consistent

304 with impaired eNOS activity are reductions in vessel number (Figure 8E), vessel surface area

305 (Figure 8H), number of end points (Figure 8K), branching index (Figure 8L), and increased

306 lacunarity (Figure $8 \mathrm{~J}$ ). These parameters are all suggestive of diabetes-induced retinal vessel

307 dysfunction in the eSWELL1 KO mice, consistent with the loss of eNOS activity that is expected

308 when insulin signaling is compromised(48) (49). Notably, both WT and eSWELL1 KO mice were

309 found to be equally glucose intolerant and insulin-resistant (Figure 8-Figure Supplement 3),

310 indicating that these differences in microvascular dysfunction were not due increased

311 hyperglycemia and more severe diabetes in eSWELL1 KO mice. Taken together, our findings

312 reveal that SWELL1 is highly expressed in endothelium and functionally encodes endothelial

313 VRAC. SWELL1 regulates ERK, AKT-eNOS, and mTOR signaling, forms a SWELL1-GRB2-

314 Cav1-eNOS signaling complex, and regulates vascular function in vivo. 

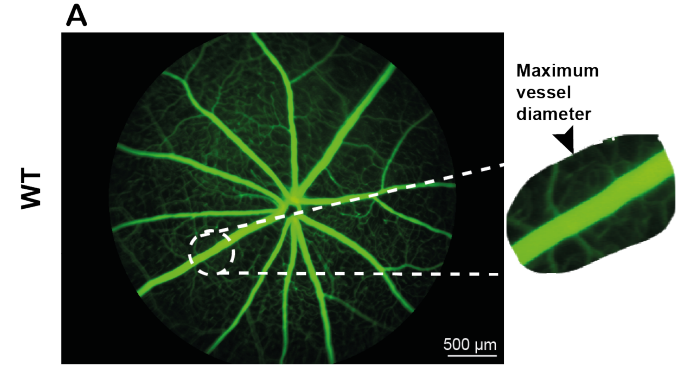

$$
\text { B }
$$
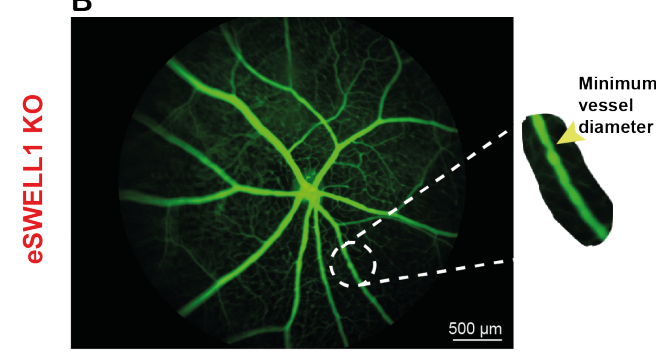
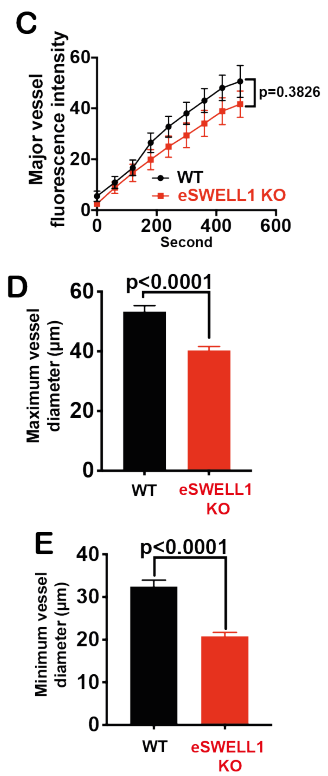
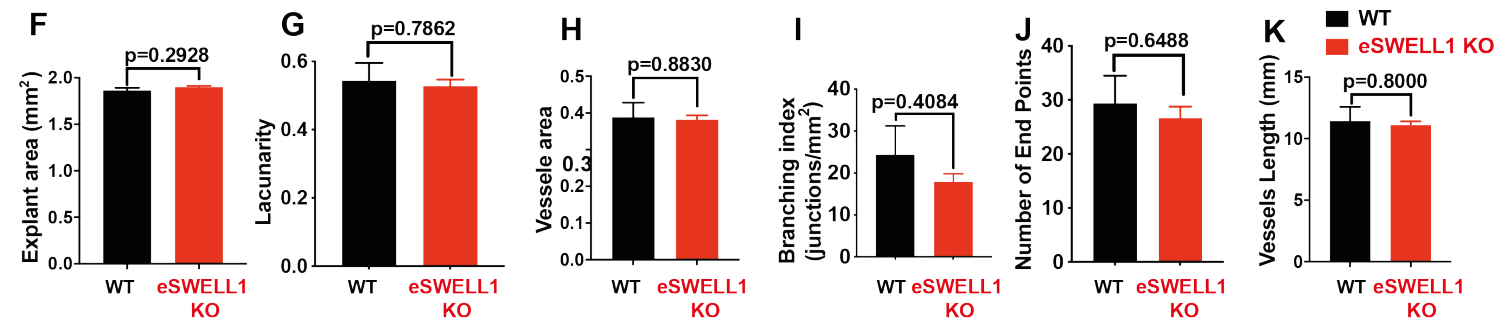

Figure 8- Figure Supplement-1. Endothelium-specific SWELL1 KO mice exhibit mild retinal microvascular disease at baseline

Representative fluorescein retinal angiograms of 13 week old WT (A) and eSWELL1 KO (B) mice raised on a regular diet. Inset shows magnified view of retinal vessels. (C-E) Quantification of major vessel fluorescence intensity over time after i.p. fluorescein injection (C), maximum vessel diameter (D), and minimum vessel diameter $(E)$ in WT $(n=11$ mice $)$ and eSWELL1 KO $(n=11$ mice). (F-K) Quantification of explant area (F), Lacunarity (G), Vessel area (H), Branching Index (I), Number of end points (J); and vessel length (K) in WT and eSWELL1 KO mice. Statistical significance between the indicated values calculated using a two-tailed Student's t-test. P-values are illustrated on figures. Error bars represent 
A
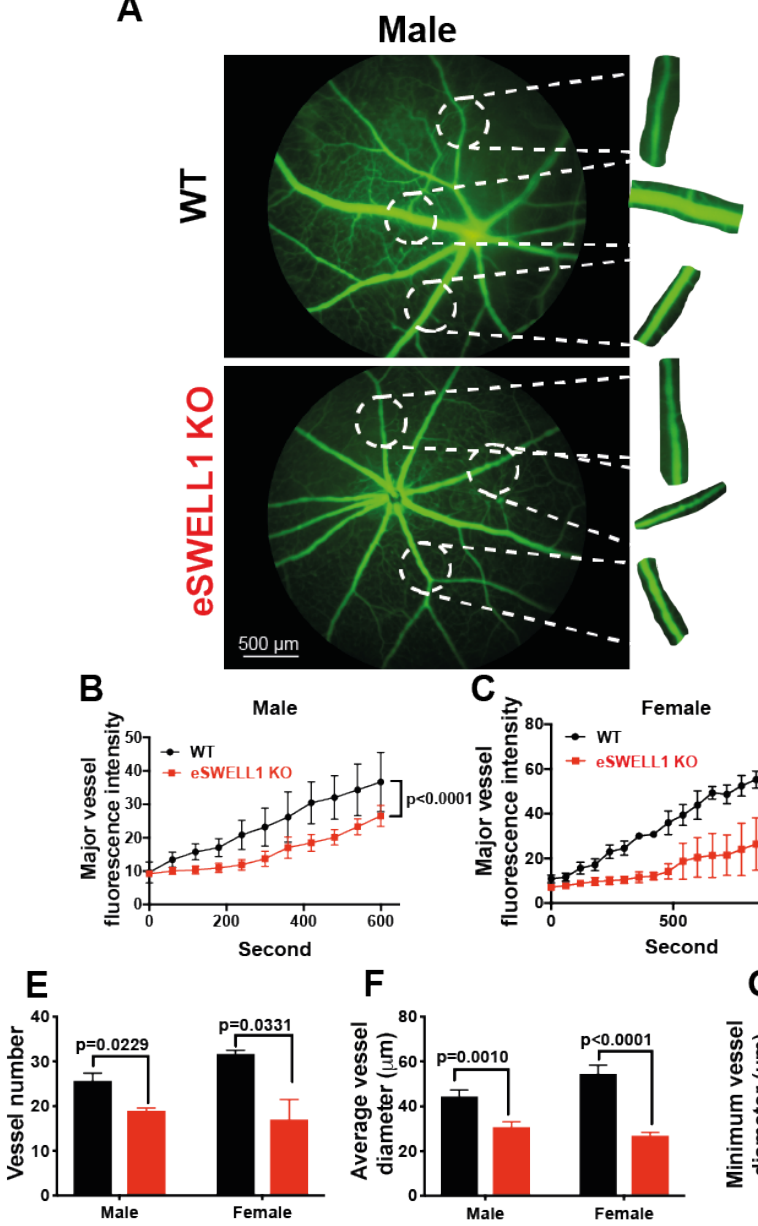

$\mathbf{F}$

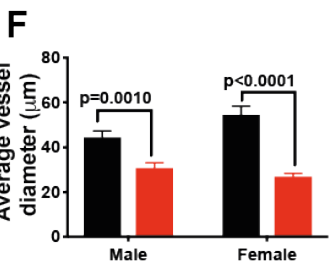

'

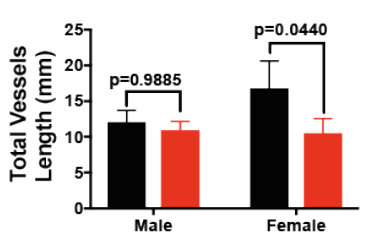

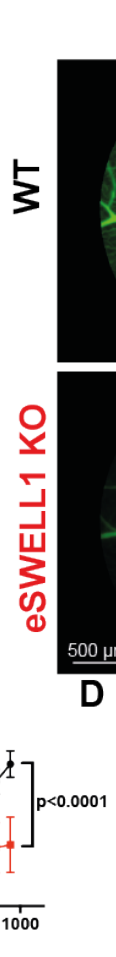

G

\section{Female}
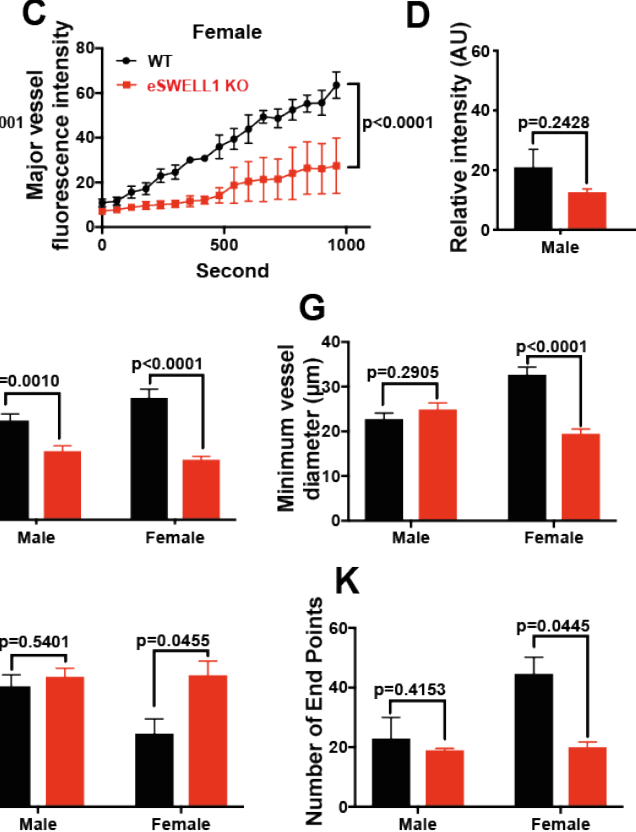

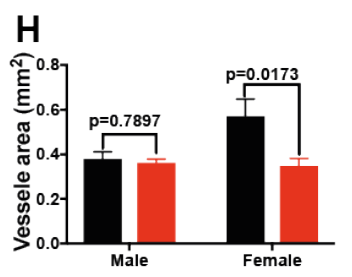

$\mathbf{L}$

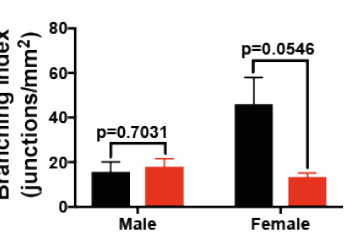

Figure 8. Endothelium-specific SWELL1 KO mice exhibit exacerbated impairments retinal microvascular disease in the setting of Type 2 diabetes

A, Representative fluorescein retinal angiograms of WT (top) and eSWELL1 KO (bottom) male (left) and female (right) mice raised on a high-fat high sucrose (HFHS) diet. Inset shows magnified view of retinal vessels. Quantification of major vessel fluorescence intensity over time after i.p. fluorescein injection in (B) male $(n=3)$ and (C) female $(n=3)$ WT and eSWELL1 KO mice. (D-K) Quantification of total retinal vessel intensity (D), Total vessel number (E); Vessel diameter (F); Minimum vessel diameter (G); Vessel area $(\mathbf{H})$; Total vessel length (I); Lacunarity (J); Number of end points (K); and Branching index (L) of retinal vessels in WT and eSWELL1 KO mice. Statistical significance between the indicated values calculated using 2-way Anova for B\&C and D-L using a two-tailed Student's t-test. P-values are illustrated on figures. Error bars represent mean \pm s.e.m. 
A

Male

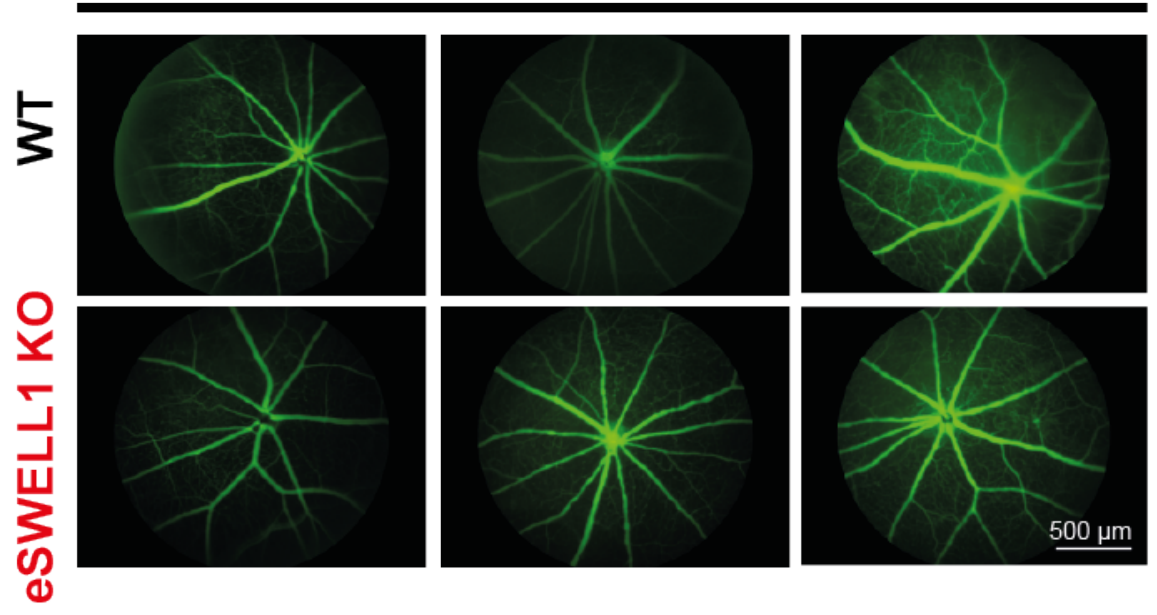

B

Female

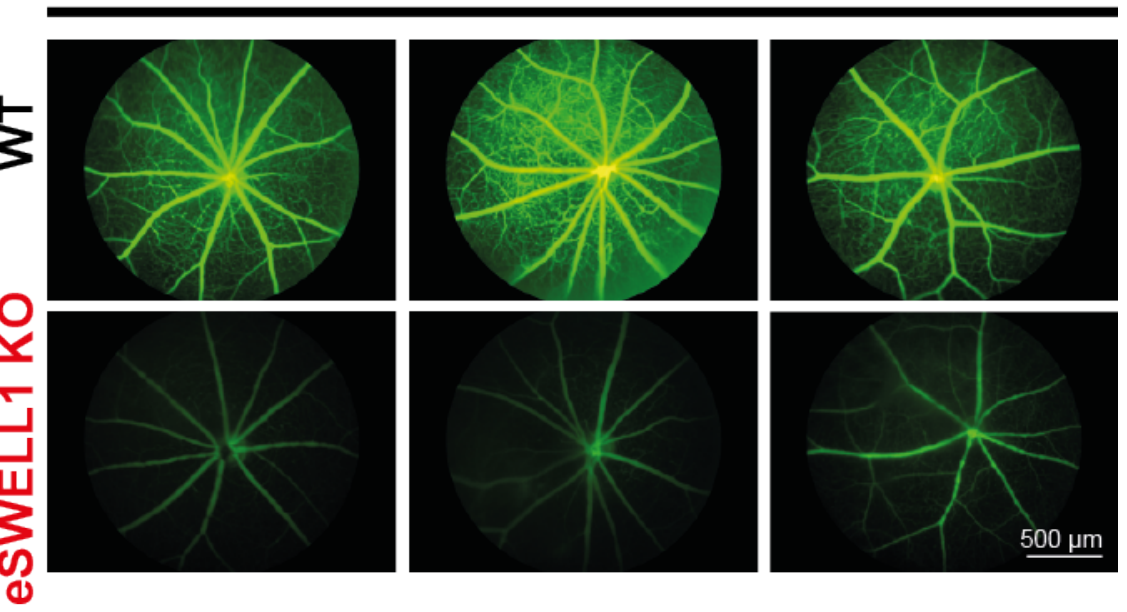

342 Figure 8- Figure Supplement-2. Endothelium-specific SWELL1 KO mice exhibit exacerbated 343 impairments retinal microvascular disease in the setting of Type 2 diabetes

344 Three representative fluorescein retinal angiograms of WT and eSWELL1 KO from male (A) and female (B) mice raised on a high-fat high sucrose (HFHS) diet for 10 months. Scale bar is $500 \mu \mathrm{m}$. 
GTT

ITT
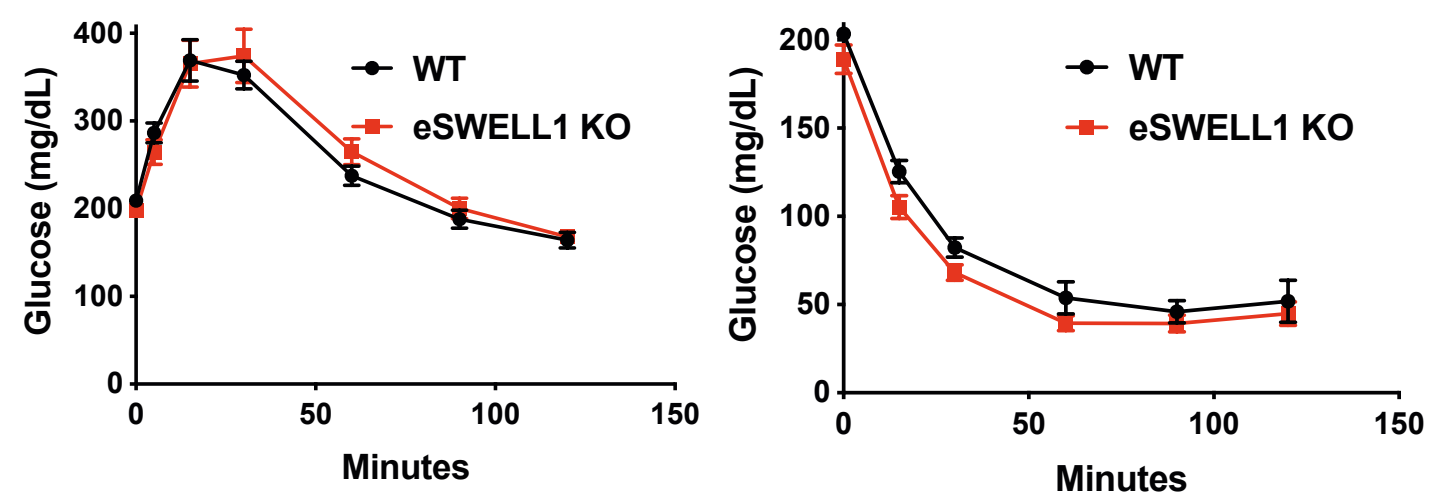

347

348

Figure 8- Figure Supplement-3. Glucose tolerance (GTT) and insulin tolerance (ITT) are not altered fat high sucrose diet for 10 months. 


\section{Discussion}

353 Our findings demonstrate that the SWELL1-LRRC8 heterohexamer functionally encodes

354 endothelial VRAC, whereby SWELL1-LRRC8 associates with GRB2 and Cav1 and positively

355 regulates PI3K-AKT-eNOS and ERK1/2 signaling. Under basal conditions and with stretch,

356 SWELL1 depletion in HUVECs reduces pAKT2, pAKT1, p-eNOS and pERK1/2. These data

357 reveal that SWELL1 mediated PI3K-AKT signaling is conserved in endothelium, similar to

358 previous observations in adipocytes(19), and in turn positively regulates eNOS expression and

359 activity. Consistent with this mechanism, endothelial-targeted SWELL1 ablation in vivo

360 predisposes to microvascular dysfunction in the setting of Type 2 diabetes, and to hypertension

361 in response to angiotensin-II infusion. These results are in line with the notion that SWELL1

362 depleted endothelium contributes to an insulin-resistant state in which impaired PI3K-AKT-

363 eNOS signaling results in a propensity for vascular dysfunction(24, 25). Insulin-mediated

364 regulation of NO is physiologically(50-52) and pathophysiologically(53) important, as NO has

365 vasodilatory $(54,55)$, anti-inflammatory(56), antioxidant(57), and antiplatelet effects(58-61).

366 Indeed, impaired NO-mediated vascular reactivity is a predictor of future adverse cardiac

367 events(62) and portends increased risk of atherosclerosis(63). Consistent with these NO-

368 mediated effects, the RNA sequencing data derived from SWELL1 KD HUVECs revealed

369 enrichment in inflammatory, cell adhesion, and proliferation pathways (GADD45, IL-8, mTOR,

370 TREM1 signaling) that may arise from SWELL1-mediated dysregulation of eNOS activity.

372 In addition to reductions in AKT-eNOS signaling, SWELL1 depletion in HUVECs also reduced

373 ERK1/2 signaling. This decrease in pERK1/2 suggests impaired MAPK signaling which is

374 connected to the insulin receptor by GRB2-SOS. Indeed, we also found that SWELL1 and

375 GRB2 interact in HUVECs, and this may provide the molecular mechanism for the observed

376 defect in ERK signaling. Interestingly, GRB2-MAPK signaling is thought to promote

377 angiogenesis, migration and proliferation(64), so reductions in ERK1/2 signaling would be 
378 predicted to inhibit these processes. Instead, aortic explants from SWELL1 KO mice exhibit

379 augmented tube formation - indicative of pro-angiogenesis. This pro-angiogenesis, pro-

380 migration cellular phenotype observed upon SWELL1 depletion might be explained by increases

381 pS6K and p70 signaling, as observed in HUVECs, and suggestive of mTORC1

382 hyperactivation(47), in addition to the observed increases in VEGFA and CD31 expression.

383 Future studies will further delineate the molecular mechanisms of SWELL1 modulation of

384 insulin-GRB2-AKT/ERK1/2 and mTOR signaling in endothelial cells.

The phenotype of endothelial targeted SWELL1 KO (eSWELL1 KO) mice are consistent with a

and exacerbated hypertension in response to chronic angiotension infusion, suggesting a

eSWELL1 KO mice raised on a regular diet, with some evidence of microvascular disease.

391 However, both retinal blood flow and retinal vessel morphology are markedly impaired in obese-

392 T2D, insulin resistant eSWELL1 KO mice raised on a high fat high sucrose diet compared to

393 controls. This is consistent with a synergistic role of endothelial SWELL1 ablation and

394 T2D/obesity in the pathogenesis of vascular disease. Indeed, our results suggest that

395 reductions in SWELL1 signaling may contribute to impaired vascular function observed in

396 humans in response to insulin and/or shear stress in the setting of obesity (65-68) and insulin-

397 resistance (69).

400 AUTHOR CONTRIBUTIONS

401 Conceptualization, R.S.; Methodology, A.A., C.T., O.A., A.K., U.F., J.M., M. E-H., M.R., C.E.G., 402 R.A.M., L.X., S.G., R.F.M., C.K., A.S., J.A., R.S.; Formal Analysis, A.A., R.S., C.T., J.M., A.K., 403 L.X., S.G, R.A.M., U.F., C.K., C.E.G.; Investigation, R.S., A.A., L.X., J.M., A.K., S.G, C.T., C.K., 
404 C.G., R.M.; Resources, R.S.; Writing - Original Draft, R.S., Writing - Review \& Editing, R.S.,

405 C.T., A.A., L.X., S.G., C.E.G.; Visualization, R.S., A.A., C.T., J.M., C.K., L.X., S.G, R.A.M.,

406 C.E.G.; Supervision, A.S., R.F.M., C.E.G, R.S.; Funding Acquisition, R.S.

407

408 DATA AVAILABILITY

409 All raw RNA sequencing data will be uploaded to Gene Expression Omnibus (GEO). All other

410 data will be made available upon reasonable request.

\section{ACKNOWLEDGMENTS}

413 RNA-Seq data presented herein were obtained at the Genomics Division of the lowa Institute of

414 Human Genetics. This work was supported by grants from the NIH/NHLBI R01 HL125436

415 (C.E.G), NIH/NHLBI 5R00HL125683 (A.S), Cancer Research Foundation Young Investigator

416 Award (A.S.), NIH NIDDK 1R01DK106009 (R.S.), the Roy J. Carver Trust (R.S.), UIHC Center

417 for Hypertension Research Pilot and Feasibility Grant and from King Abdullah International

418 Medical Research Center (KAIMRC) grant RA17-014-A (A. A.). We thank Dr. Rithwick

419 Rajagopal for insightful reading of the manuscript. 
423 1. Cahill PA, and Redmond EM. Vascular endothelium - Gatekeeper of vessel health. Atherosclerosis. 2016;248:97-109.

2. Gerhold KA, and Schwartz MA. Ion Channels in Endothelial Responses to Fluid Shear Stress. Physiology (Bethesda). 2016;31(5):359-69.

Sonkusare SK, Bonev AD, Ledoux J, Liedtke W, Kotlikoff MI, Heppner TJ, et al. Elementary $\mathrm{Ca} 2+$ signals through endothelial TRPV4 channels regulate vascular function. Science. 2012;336(6081):597-601.

4. $\quad$ Earley S, Pauyo T, Drapp R, Tavares MJ, Liedtke W, and Brayden JE. TRPV4dependent dilation of peripheral resistance arteries influences arterial pressure. Am J Physiol Heart Circ Physiol. 2009;297(3):H1096-102.

5. Zhang DX, Mendoza SA, Bubolz AH, Mizuno A, Ge ZD, Li R, et al. Transient receptor potential vanilloid type 4-deficient mice exhibit impaired endothelium-dependent relaxation induced by acetylcholine in vitro and in vivo. Hypertension. 2009;53(3):532-8.

6. Mendoza SA, Fang J, Gutterman DD, Wilcox DA, Bubolz AH, Li R, et al. TRPV4mediated endothelial $\mathrm{Ca2}+$ influx and vasodilation in response to shear stress. Am J Physiol Heart Circ Physiol. 2010;298(2):H466-76.

7. Rode B, Shi J, Endesh N, Drinkhill MJ, Webster PJ, Lotteau SJ, et al. Piezo1 channels sense whole body physical activity to reset cardiovascular homeostasis and enhance performance. Nat Commun. 2017;8(1):350.

8. Li J, Hou B, Tumova S, Muraki K, Bruns A, Ludlow MJ, et al. Piezo1 integration of vascular architecture with physiological force. Nature. 2014;515(7526):279-82.

9. Coste B, Mathur J, Schmidt M, Earley TJ, Ranade S, Petrus MJ, et al. Piezo1 and Piezo2 are essential components of distinct mechanically activated cation channels. Science. 2010;330(6000):55-60.

10. Nilius $B$, and Droogmans $G$. Ion channels and their functional role in vascular endothelium. Physiol Rev. 2001;81(4):1415-59.

11. Barakat Al, Leaver EV, Pappone PA, and Davies PF. A flow-activated chloride-selective membrane current in vascular endothelial cells. Circ Res. 1999;85(9):820-8.

12. Sawada A, Takihara Y, Kim JY, Matsuda-Hashii Y, Tokimasa S, Fujisaki H, et al. A congenital mutation of the novel gene LRRC8 causes agammaglobulinemia in humans. J Clin Invest. 2003;112(11):1707-13.

13. Kubota K, Kim JY, Sawada A, Tokimasa S, Fujisaki H, Matsuda-Hashii Y, et al. LRRC8 involved in $B$ cell development belongs to a novel family of leucine-rich repeat proteins. FEBS Lett. 2004;564(1-2):147-52.

14. Kumar L, Chou J, Yee CS, Borzutzky A, Vollmann EH, von Andrian UH, et al. Leucinerich repeat containing $8 \mathrm{~A}(\mathrm{LRRC} 8 \mathrm{~A})$ is essential for T lymphocyte development and function. J Exp Med. 2014;211(5):929-42.

15. Abascal F, and Zardoya R. LRRC8 proteins share a common ancestor with pannexins, and may form hexameric channels involved in cell-cell communication. Bioessays. 2012;34(7):551-60.

16. Qiu Z, Dubin AE, Mathur J, Tu B, Reddy K, Miraglia LJ, et al. SWELL1, a Plasma Membrane Protein, Is an Essential Component of Volume-Regulated Anion Channel. Cell. 2014;157(2):447-58.

17. Voss FK, Ullrich F, Munch J, Lazarow K, Lutter D, Mah N, et al. Identification of LRRC8 heteromers as an essential component of the volume-regulated anion channel VRAC. Science. 2014;344(6184):634-8.

18. Syeda R, Qiu Z, Dubin AE, Murthy SE, Florendo MN, Mason DE, et al. LRRC8 Proteins Form Volume-Regulated Anion Channels that Sense lonic Strength. Cell. 2016;164(3):499-511. 
501

502

503

504

505

506

507

508

509

510

511

512

513

514

515

516

517

518

519

520

521

19. Zhang Y, Xie L, Gunasekar SK, Tong D, Mishra A, Gibson WJ, et al. SWELL1 is a regulator of adipocyte size, insulin signalling and glucose homeostasis. Nature cell biology. 2017;19(5):504-17.

20. Xie L, Zhang Y, Gunasekar S, Mishra A, Cao L, and Sah R. Induction of adipose and hepatic SWELL1 expression is required for maintaining systemic insulin-sensitivity in obesity. Channels. 2017:0.

21. Gunasekar SK, Xie L, and Sah R. SWELL signalling in adipocytes: can fat 'feel' fat? Adipocyte. 2019;8(1):223-8.

22. Hu Z, Xiong $Y$, Han X, Geng $C$, Jiang B, Huo $Y$, et al. Acute mechanical stretch promotes eNOS activation in venous endothelial cells mainly via PKA and Akt pathways. PLoS One. 2013;8(8):e71359.

23. Janus A, Szahidewicz-Krupska E, Mazur G, and Doroszko A. Insulin Resistance and Endothelial Dysfunction Constitute a Common Therapeutic Target in Cardiometabolic Disorders. Mediators Inflamm. 2016;2016:3634948.

24. Kearney MT, Duncan ER, Kahn M, and Wheatcroft SB. Insulin resistance and endothelial cell dysfunction: studies in mammalian models. Exp Physiol. 2008;93(1):15863.

25. Muniyappa R, and Sowers JR. Role of insulin resistance in endothelial dysfunction. Rev Endocr Metab Disord. 2013;14(1):5-12.

26. Barakat Al. Responsiveness of vascular endothelium to shear stress: potential role of ion channels and cellular cytoskeleton (review). Int J Mol Med. 1999;4(4):323-32.

27. Kang C, Xie L, Gunasekar SK, Mishra A, Zhang Y, Pai S, et al. SWELL1 is a glucose sensor regulating beta-cell excitability and systemic glycaemia. Nat Commun. 2018;9(1):367.

28. Stuhlmann T, Planells-Cases R, and Jentsch TJ. LRRC8/VRAC anion channels enhance beta-cell glucose sensing and insulin secretion. Nat Commun. 2018;9(1):1974.

29. Wang R, Lu Y, Gunasekar S, Zhang Y, Benson CJ, Chapleau MW, et al. The volumeregulated anion channel (LRRC8) in nodose neurons is sensitive to acidic $\mathrm{pH}$. $\mathrm{JCl}$ Insight. 2017;2(5):e90632.

30. Luck JC, Puchkov D, Ullrich F, and Jentsch TJ. LRRC8/VRAC anion channels are required for late stages of spermatid development in mice. J Biol Chem.

2018;293(30):11796-808.

31. Duncan ER, Crossey PA, Walker S, Anilkumar N, Poston L, Douglas G, et al. Effect of endothelium-specific insulin resistance on endothelial function in vivo. Diabetes. 2008;57(12):3307-14.

32. Morello F, Perino A, and Hirsch E. Phosphoinositide 3-kinase signalling in the vascular system. Cardiovasc Res. 2009;82(2):261-71.

33. Ju H, Zou R, Venema VJ, and Venema RC. Direct interaction of endothelial nitric-oxide synthase and caveolin-1 inhibits synthase activity. J Biol Chem. 1997;272(30):18522-5.

34. Venema VJ, Zou R, Ju H, Marrero MB, and Venema RC. Caveolin-1 detergent solubility and association with endothelial nitric oxide synthase is modulated by tyrosine phosphorylation. Biochemical and biophysical research communications. 1997;236(1):155-61.

35. Goligorsky MS, Li H, Brodsky S, and Chen J. Relationships between caveolae and eNOS: everything in proximity and the proximity of everything. Am J Physiol Renal Physiol. 2002;283(1):F1-10.

36. Salameh A, Galvagni F, Bardelli M, Bussolino F, and Oliviero S. Direct recruitment of CRK and GRB2 to VEGFR-3 induces proliferation, migration, and survival of endothelial cells through the activation of ERK, AKT, and JNK pathways. Blood. 2005;106(10):342331. 
37. Nassoy $\mathrm{P}$, and Lamaze $\mathrm{C}$. Stressing caveolae new role in cell mechanics. Trends in cell biology. 2012;22(7):381-9.

38. Sinha B, Koster D, Ruez R, Gonnord P, Bastiani M, Abankwa D, et al. Cells respond to mechanical stress by rapid disassembly of caveolae. Cell. 2011;144(3):402-13.

39. Sedding DG, Hermsen J, Seay U, Eickelberg O, Kummer W, Schwencke C, et al. Caveolin-1 facilitates mechanosensitive protein kinase $B(\mathrm{Akt})$ signaling in vitro and in vivo. Circ Res. 2005;96(6):635-42.

40. Trouet D, Hermans D, Droogmans G, Nilius B, and Eggermont J. Inhibition of volumeregulated anion channels by dominant-negative caveolin-1. Biochemical and biophysical research communications. 2001;284(2):461-5.

41. Trouet D, Nilius B, Jacobs A, Remacle C, Droogmans G, and Eggermont J. Caveolin-1 modulates the activity of the volume-regulated chloride channel. J Physiol. 1999;520 Pt 1:113-9.

42. Browe DM, and Baumgarten CM. Stretch of beta 1 integrin activates an outwardly rectifying chloride current via FAK and $\mathrm{Src}$ in rabbit ventricular myocytes. J Gen Physiol. 2003;122(6):689-702.

43. Browe DM, and Baumgarten CM. EGFR kinase regulates volume-sensitive chloride current elicited by integrin stretch via PI-3K and NADPH oxidase in ventricular myocytes. J Gen Physiol. 2006;127(3):237-51.

44. Nakao M, Ono K, Fujisawa S, and lijima T. Mechanical stress-induced Ca2+ entry and $\mathrm{Cl}$ - current in cultured human aortic endothelial cells. Am J Physiol. 1999;276(1 Pt 1):C238-49.

45. Romanenko VG, Davies PF, and Levitan I. Dual effect of fluid shear stress on volumeregulated anion current in bovine aortic endothelial cells. Am J Physiol Cell Physiol. 2002;282(4):C708-18.

46. Fulton D, Fontana J, Sowa G, Gratton JP, Lin M, Li KX, et al. Localization of endothelial nitric-oxide synthase phosphorylated on serine 1179 and nitric oxide in Golgi and plasma membrane defines the existence of two pools of active enzyme. $J$ Biol Chem. 2002;277(6):4277-84.

47. Ding Y, Shan L, Nai W, Lin X, Zhou L, Dong X, et al. DEPTOR Deficiency-Mediated mTORc1 Hyperactivation in Vascular Endothelial Cells Promotes Angiogenesis. Cell Physiol Biochem. 2018;46(2):520-31.

48. Brooks SE, Gu X, Samuel S, Marcus DM, Bartoli M, Huang PL, et al. Reduced severity of oxygen-induced retinopathy in eNOS-deficient mice. Invest Ophthalmol Vis Sci. 2001;42(1):222-8.

49. Kondo T, Vicent D, Suzuma K, Yanagisawa M, King GL, Holzenberger M, et al. Knockout of insulin and IGF-1 receptors on vascular endothelial cells protects against retinal neovascularization. J Clin Invest. 2003;111(12):1835-42.

50. Zeng G, and Quon MJ. Insulin-stimulated production of nitric oxide is inhibited by wortmannin. Direct measurement in vascular endothelial cells. J Clin Invest. 1996;98(4):894-8.

51. Zeng G, Nystrom FH, Ravichandran LV, Cong LN, Kirby M, Mostowski H, et al. Roles for insulin receptor, PI3-kinase, and Akt in insulin-signaling pathways related to production of nitric oxide in human vascular endothelial cells. Circulation. 2000;101(13):1539-45.

52. Montagnani M, Ravichandran LV, Chen H, Esposito DL, and Quon MJ. Insulin receptor substrate-1 and phosphoinositide-dependent kinase-1 are required for insulin-stimulated production of nitric oxide in endothelial cells. Molecular endocrinology. 2002;16(8):193142.

53. Steinberg HO, Chaker H, Leaming R, Johnson A, Brechtel G, and Baron AD. Obesity/insulin resistance is associated with endothelial dysfunction. Implications for the syndrome of insulin resistance. J Clin Invest. 1996;97(11):2601-10. 
601

602

603

604

605

606

607

608

609

610

611

612

613

614

615

616

617

618

619
54. Quillon A, Fromy B, and Debret R. Endothelium microenvironment sensing leading to nitric oxide mediated vasodilation: a review of nervous and biomechanical signals. Nitric Oxide. 2015;45:20-6.

55. Palmer RM, Ferrige AG, and Moncada S. Nitric oxide release accounts for the biological activity of endothelium-derived relaxing factor. Nature. 1987;327(6122):524-6.

56. Kataoka C, Egashira K, Inoue S, Takemoto M, Ni W, Koyanagi M, et al. Important role of Rho-kinase in the pathogenesis of cardiovascular inflammation and remodeling induced by long-term blockade of nitric oxide synthesis in rats. Hypertension. 2002;39(2):245-50.

57. Clapp BR, Hingorani AD, Kharbanda RK, Mohamed-Ali V, Stephens JW, Vallance P, et al. Inflammation-induced endothelial dysfunction involves reduced nitric oxide bioavailability and increased oxidant stress. Cardiovasc Res. 2004;64(1):172-8.

58. Schafer A, Widder J, Eigenthaler M, Ertl G, and Bauersachs J. Reduced basal nitric oxide bioavailability and platelet activation in young spontaneously hypertensive rats. Biochem Pharmacol. 2004;67(12):2273-9.

59. Schafer A, Wiesmann F, Neubauer S, Eigenthaler M, Bauersachs J, and Channon KM. Rapid regulation of platelet activation in vivo by nitric oxide. Circulation. 2004;109(15):1819-22.

60. Radomski MW, Palmer RM, and Moncada S. The role of nitric oxide and cGMP in platelet adhesion to vascular endothelium. Biochemical and biophysical research communications. 1987;148(3):1482-9.

61. Radomski MW, Palmer RM, and Moncada S. Endogenous nitric oxide inhibits human platelet adhesion to vascular endothelium. Lancet. 1987;2(8567):1057-8.

62. Schachinger V, Britten MB, and Zeiher AM. Prognostic impact of coronary vasodilator dysfunction on adverse long-term outcome of coronary heart disease. Circulation. 2000;101(16):1899-906.

63. Bugiardini R, Manfrini O, Pizzi C, Fontana F, and Morgagni G. Endothelial function predicts future development of coronary artery disease: a study of women with chest pain and normal coronary angiograms. Circulation. 2004;109(21):2518-23.

64. Z Zhao J, Wang W, Ha CH, Kim JY, Wong C, Redmond EM, et al. Endothelial Grb2associated binder 1 is crucial for postnatal angiogenesis. Arterioscler Thromb Vasc Biol. 2011;31(5):1016-23.

65. Arcaro G, Zamboni M, Rossi L, Turcato E, Covi G, Armellini F, et al. Body fat distribution predicts the degree of endothelial dysfunction in uncomplicated obesity. Int J Obes Relat Metab Disord. 1999;23(9):936-42.

66. Tack CJ, Ong MK, Lutterman JA, and Smits P. Insulin-induced vasodilatation and endothelial function in obesity/insulin resistance. Effects of troglitazone. Diabetologia. 1998;41(5):569-76.

67. Westerbacka J, Vehkavaara S, Bergholm R, Wilkinson I, Cockcroft J, and Yki-Jarvinen $\mathrm{H}$. Marked resistance of the ability of insulin to decrease arterial stiffness characterizes human obesity. Diabetes. 1999;48(4):821-7.

68. Williams IL, Chowienczyk PJ, Wheatcroft SB, Patel A, Sherwood R, Momin A, et al. Effect of fat distribution on endothelial-dependent and endothelial-independent vasodilatation in healthy humans. Diabetes Obes Metab. 2006;8(3):296-301.

69. Murphy C, Kanaganayagam GS, Jiang B, Chowienczyk PJ, Zbinden R, Saha M, et al. Vascular dysfunction and reduced circulating endothelial progenitor cells in young healthy UK South Asian men. Arterioscler Thromb Vasc Biol. 2007;27(4):936-42. 
Figure legends

\section{Figure.1. SWELL1 mediates VRAC currents in human umbilical vein endothelial cells}

623 (HUVECs). A, SWELL1 western blot in HUVECs transduced with adenovirus expressing a short 624 hairpin RNA directed to Swell1 (Ad-shSWELL1) compared to control scrambled short hairpin 625 RNA (Ad-shSCR). GAPDH is used as loading control. B, Immunofluorescence staining of the 626 HUVECs transduced with Ad-shSWELL1 and Ad-shSCR. C, Current-time relationship of VRAC 627 (hypotonic, $210 \mathrm{mOsm}$ ) in Ad-shSCR transduced HUVEC and co-application of $10 \mu \mathrm{M}$ DCPIB.

628 D, Representative current traces upon hypotonic activation (left) during voltage steps (from -100 629 to $+100 \mathrm{mV}$, shown in inset) and inhibition by DCPIB (right). E, Current-voltage relationship of 630 VRAC during voltage ramps from $-100 \mathrm{mV}$ to $+100 \mathrm{mV}$ after hypotonic swelling in HUVECs 631 transduced with Ad-shSCR and Ad-shSWELL1. F. Mean current outward and inward densities 632 at +100 and $-100 \mathrm{mV}(\mathrm{n}, \mathrm{sh}-\mathrm{SCR}=4$ cells; $\mathrm{n}$,shSWELL1 $=6$ cells $)$. Data are shown as mean \pm s.e.m. ${ }^{*} \mathrm{p}$ $633<0.05 ;{ }^{* *} p<0.01$; unpaired t-test for $\mathbf{F}$.

Figure 2. SWELL1 regulates PI3K-AKT-eNOS, ERK and mTOR signaling in endothelium.

635 (A), Western blots of SWELL1, pAkt2, pAkt1, Akt2, Akt1, pErk1/2, Erk1/2, p-eNOS, eNOS, pS6K ribosomal protein, S6K ribosomal protein, GAPDH, and $\beta$-Actin in Ad-shSCR and AdshSWELL1 transduced HUVECS under basal conditions. Quantification of SWELL1/ $\beta$-Actin (B), p-eNOS/ $\beta$-actin, p-eNOS/Total eNOS (C), pAkt2/ $\beta$-actin, pAkt2/Total Akt2 (D), pAkt1/GAPDH, pAkt1/Total Akt1 (E), pERK1/2 /GAPDH, pErk1/2 /Total Erk1/2 (F), pS6

640 ribosomal protein/GAPDH, and pS6K ribosomal protein/Total S6K ribosomal protein (G). N=6 641 independent experiments. Significance between the indicated groups in all blots were calculated 642 using a two-tailed Student's t-test. P-values are illustrated on figures. Error bars represent mean $643 \pm$ s.e.m. 
645 Figure 3. SWELL1 interacts with Grb2, Cav1 and eNOS in human endothelium

646 A, GRB2 immunoprecipitation from Ad-shSCR and Ad-shSWELL1 transduced HUVECs and

647 immunoblot with SWELL1, Cav1 and GRB2 antibodies. Densitometry values for GRB2 co-

648 immunoprecipitated SWELL1 (SWELL1/GRB2) and GRB2 co-immunoprecipitated Cav1

649 (Cav1/GRB2). GAPDH serves as loading control for input samples. (B) GRB2

650 immunoprecipitation from Ad-shSCR and Ad-shSWELL1 transduced HUVECs and immunoblot

651 with SWELL1, eNOS, Cav1 and GRB2 antibodies. Insulin-stimulation with 100 nM insulin for 10

652 minutes. Densitometry values for GRB2 co-immunoprecipitated eNOS (eNOS/GRB2).

653 Representative blots from 3 independent experiments. (C) Representative endogenous

654 SWELL1 and eNOS immunofluorescence staining in Ad-shSCR and Ad-shSWELL1 transduced

655 HUVECs. Representative image from 6 independent experiments. (D-E) Quantification of

656 SWELL1 $(\mathbf{D}, \mathrm{n}=6)$ and eNOS $(\mathbf{E}, \mathrm{n}=6)$ immunofluorescence staining upon SWELL1 KD.

657 Evidence of SWELL1-eNOS colocalization (C, insets) in plasma membrane (i) and perinuclear

658 regions (ii). Significance between the indicated groups are calculated using a two-tailed

659 Student's t-test. P-values are indicated on figures. Error bars represent mean \pm s.e.m.

660

Figure 4. SWELL1 is required for intact stretch-induced AKT-eNOS signaling

662 A, Western blot of SWELL1, pAKT1, pAKT2, pERK1/2 in response to 30 minutes of $0.1 \%$ and

$6635 \%$ static stretch in Ad-shSCR and Ad-shSWELL1 transduced HUVECs. GAPDH is used as a

664 loading control. (B-D) Densitometry quantification from A of pAKT1 (B), pAKT2 (C) and pErk1/2

665 (D). (E) Western blot of peNOS, SWELL1, in response to 5\% static stretching for 5, 60 and 180 666 min in Ad-shSCR and Ad-shSWELL1 transduced HUVECs. (F) Densitometry quantification from 667 E of eNOS. GAPDH is used as a loading control. Significance between the indicated groups in 
all blots were calculated using a two-tailed Student's t-test. P-values are illustrated on figures.

Error bars represent mean \pm s.e.m.

Figure 5. Endothelium-specific SWELL1 KO mice exhibit enhanced tube formation from

A, Strategy for endothelium targeted SWELL1 ablation to generate eSWELL1 KO. (B) Isolation

674 of murine primary endothelial cells from WT and eSWELL1 KO using tdTomato reporter mice.

675 C-D, Current-voltage relationships of VRAC in isotonic (Iso, $300 \mathrm{mOsM}$ ) and hypotonic (Hypo,

$210 \mathrm{mOsm}$ ) solution in response to voltage ramps from -100 to $+100 \mathrm{mV}$ over $500 \mathrm{~ms}$ in WT (C)

and $\mathrm{KO}(\mathrm{D})$ primary murine endothelial cells. DCPIB $(10 \mu \mathrm{M})$ inhibition in C (WT). (E) Mean

outward $(+100 \mathrm{mV})$ and inward $(-100 \mathrm{mV})$ currents from WT $(\mathrm{n}=3$ cells) and eSWELL1 KO $(\mathrm{n}=3$

cells). (F) Ex-vivo aorta sprouting assay performed in aortic rings isolated from WT and

eSWELL1 KO mice and cultured in FGM media for 3 days at 37oC. Immunofluoresence staining

681 with antibodies to SWELL1 (green), CD31 (red), SWELL1+CD31+ (Merge) and bright field

682 images show endothelial cell tubes sprouting from WT and eSWELL1 KO aortic rings (black

683 arrow heads). (G-I) Quantification of SWELL1 immunofluorescence signal (G, WT = 15, KO =

684 15), number of tip cells $(\mathrm{H}, \mathrm{WT}=26, \mathrm{KO}=31)$, and endothelial tube length $(\mathrm{I}, \mathrm{WT}=30, \mathrm{KO}=$

$68530)$ in WT and eSWELL1 KO aortic explants. Statistical significance between the indicated

686 values calculated using a two-tailed Student's t-test. P-values are illustrated on figures. Error

687 bars represent mean \pm s.e.m. $n=3$, independent experiments.

Figure 6. RNA sequencing of Ad-shSCR and Ad-shSWELL1 transduced HUVECs.

690 A, Heatmap analysis displaying top 25 upregulated or 25 downregulated genes between

691 shSCR and shSWELL1. (B) IPA canonical pathway analysis of genes significantly regulated by 692 shSWELL1 in comparison to shSCR. $n=3$ for each group. For analysis with IPA, FPKM cutoffs 
693 of 1.5 , fold change of $\geq 1.5$, and false discovery rate $<0.05$ were utilized for significantly

694 differentially regulated genes.

695

Figure 7. Endothelial-targeted SWELL1 deletion predisposes to systolic hypertension

697 Tail-cuff systolic blood pressures of (A) male and (B) female WT ( $n=5$ males and 7 females)

698 and eSWELL1 KO ( $\mathrm{n}=5$ males and 12 females) mice. (C) Systolic blood pressures of male WT

699 ( $n=4)$ and eSWELL1 KO ( $n=5)$ mice under basal conditions and after 4 weeks of chronic

700 angiotensin II infusion. Statistical significance between the indicated values calculated using a 701 two-tailed Student's t-test. P-values are illustrated on figures. Data are shown as mean \pm s.e.m.

Figure 8. Endothelium-specific SWELL1 KO mice exhibit exacerbated impairments retinal microvascular disease in the setting of Type 2 diabetes

A, Representative fluorescein retinal angiograms of WT (top) and eSWELL1 KO (bottom) male

706 (left) and female (right) mice raised on a high-fat high sucrose (HFHS) diet. Inset shows

707 magnified view of retinal vessels. Quantification of major vessel fluorescence intensity over time

708 after i.p. fluorescein injection in $(\mathbf{B})$ male $(n=3)$ and $(\mathbf{C})$ female $(n=3)$ WT and eSWELL1 KO

709 mice. (D-K) Quantification of total retinal vessel intensity (D), Total vessel number (E); Vessel

710 diameter (F); Minimum vessel diameter (G); Vessel area (H); Total vessel length (I); Lacunarity

711 (J); Number of end points (K); and Branching index (L) of retinal vessels in WT and eSWELL1

712 KO mice. Statistical significance between the indicated values calculated using 2-way Anova for

713 B\&C and D-L using a two-tailed Student's t-test. P-values are illustrated on figures. Error bars

714 represent mean \pm s.e.m. 


\section{Supplementary Figure Legends}

717 Figure 2- Figure Supplement 1. SWELL1 regulates PI3K-AKT-eNOS, ERK and mTOR 718 signaling in endothelium.

719 (A), Western blots of SWELL1, pAkt2, pAkt1, Akt, pErk1/2, Erk1/2, p-eNOS, eNOS, pS6K 720 ribosomal protein, S6K ribosomal protein, and $\beta$-Actin in si-SCR and si-SWELL1 transduced 721 HUVECS under basal conditions. Quantification of SWELL1/ $\beta$-Actin (B), p-eNOS/ $\beta$-actin, p722 eNOS/Total eNOS (C), pAkt2/ $\beta$-actin, pAkt2/Total Akt (D), pAkt1/ $\beta$-actin, pAkt1/Total Akt (E), 723 pERK1/2 / $\beta$-actin, pErk1/2 /Total Erk1/2 (F), pS6 ribosomal protein/ $\beta$-actin, and pS6K ribosomal protein/Total S6K ribosomal protein $(\mathbf{G}) . \mathrm{N}=6$ independent experiments. Significance between the indicated groups in all blots were calculated using a two-tailed Student's t-test. Pvalues are illustrated on figures. Error bars represent mean \pm s.e.m.

Figure 3-Figure Supplement 1. SWELL1 co-localizes with eNOS and regulates eNOS expression SWELL1 (green) and eNOS (red) immunofluorescence staining of HUVEC

Figure 8- Figure Supplement 1. Endothelium-specific SWELL1 KO mice exhibit mild retinal microvascular disease at baseline 
739 ( $\mathrm{n}=11$ mice). (F-K) Quantification of explant area (F), Lacunarity $(\mathbf{G})$, Vessel area $(\mathbf{H})$,

740 Branching Index (I), Number of end points (J); and vessel length (K) in WT and eSWELL1 KO

741 mice. Statistical significance between the indicated values calculated using a two-tailed

742 Student's t-test. P-values are illustrated on figures. Error bars represent mean \pm s.e.m. Scale

743 bar is $500 \mu \mathrm{m}$.

Figure 8- Figure Supplement 2. Endothelium-specific SWELL1 KO mice exhibit

746 exacerbated impairments retinal microvascular disease in the setting of Type 2 diabetes

747 Three representative fluorescein retinal angiograms of WT and eSWELL1 KO from male (A)

748 and female (B) mice raised on a high-fat high sucrose (HFHS) diet for 10 months. Scale bar is

$749500 \mu \mathrm{m}$.

751 Figure 8- Figure Supplement 3. Glucose tolerance (GTT) and insulin tolerance (ITT) are 752 not altered in endothelium-specific SWELL1 KO mice $(n=13)$ compared to WT mice $(n=$ 753 8) raised on a high-fat high sucrose diet for 10 months. 


\section{Materials and Methods}

758

759

760

761

762

763

764

765

766

767

768

769

770

771

772

773

774

775

776

777

778

779

780

Animals: The institutional animal care and use committee of the University of lowa and Washington University School of Medicine approved all experimental procedures involving animals. All mice were housed in temperature, humidity and light controlled environment and allowed water access and food. Both male and female Swell1 $1^{f / f l}(1,2)(\mathrm{WT}), C D H 5^{\text {cre }}$; Swell1 $1^{f / f t}$ (eSWELL1 KO) mice were generated and used in these studies. CHD5 ${ }^{\mathrm{Cre}}$ mice were obtained from Dr. Kaikobad Irani (University of lowa, IA). In a subset of experiments, 5-8 week old Swell $1^{f / f l}$ and $\mathrm{CDH} 5^{\text {cre }}$; Swell $1^{\text {fl/fl }}$ mice were switched to HFHS (High Fat high Sucrose rodent diet, Research Diets, Inc.,Cat \# D12331) for at least 10 months. $C D H 5^{\text {cre }}$ mice were crossed with Rosa26-tdTomato (Jax\# 007914) reporter mice to identify CDH5+ cells for primary endothelium patch-clamp studies.

Antibodies. Rabbit polyclonal anti-SWELL1 antibody was generated against the epitope QRTKSRIEQGIVDRSE (Pacific Antibodies)(1). All other primary antibodies were purchased from Cells Signaling: anti- $\beta$-actin (\#8457), Total Akt (\#4685S), Akt1 (\#2938), Akt2 (\#3063), peNOS (\#9571), Total eNOS (\#32027), p-AS160 (\#4288), p-p70 S6 Kinase (\#9205S), pS6 Ribosomal (\#5364S), GAPDH (\#5174), pErk1/2 (\#9101), Total Erk1/2 (\#9102). Anti-SWELL1 antibody was custom made as described previously $(1,2)$. Purified mouse anti-Grb2 was purchased from BD (610111) and Santa Cruz (\#sc-255). Rabbit IgG Santa Cruz (sc-2027). AntiCD31 was purchased from Thermo Fisher (MA3105).

Electrophysiology. All recordings were performed in the whole-cell configuration at room temperature, as previously described(1,2). Briefly, currents were measured with either an Axopatch 200B amplifier or a MultiClamp 700B amplifier (Molecular Devices) paired to a Digidata 1550 digitizer. Both amplifiers used pClamp 10.4 software. The intracellular solution contained (in mM): 120 L-aspartic acid, 20 CsCl, 1 MgCl , 5 EGTA, 10 HEPES, 5 MgATP, 120 
$781 \mathrm{CsOH}, 0.1 \mathrm{GTP}, \mathrm{pH} 7.2$ with $\mathrm{CsOH}$. The extracellular solution for hypotonic stimulation

782 contained (in mM): $90 \mathrm{NaCl}, 2 \mathrm{CsCl}, 1 \mathrm{MgCl}_{2}, 1 \mathrm{CaCl}_{2}, 10 \mathrm{HEPES}, 5$ glucose, 5 mannitol, pH

7837.4 with $\mathrm{NaOH}(210 \mathrm{mOsm} / \mathrm{kg})$. The isotonic extracellular solution contained the same

784 composition as above except for mannitol concentration of 105 (300 mOsm/kg). The osmolarity

785 was checked by a vapor pressure osmometer 5500 (Wescor). Currents were filtered at $10 \mathrm{kHz}$

and sampled at $100 \mu$ s interval. The patch pipettes were pulled from borosilicate glass capillary

787 tubes (WPI) by a P-87 micropipette puller (Sutter Instruments). The pipette resistance was $\sim 4-6$

$788 \mathrm{M} \Omega$ when the patch pipette was filled with intracellular solution. The holding potential was $0 \mathrm{mV}$.

789 Voltage steps (500 ms) were elicited from $0 \mathrm{mV}$ holding potential from -100 to $+100 \mathrm{mV}$ in 20

$790 \mathrm{mV}$ increments every $0.6 \mathrm{~s}$. Voltage ramps from -100 to $+100 \mathrm{mV}$ (at $0.4 \mathrm{mV} / \mathrm{ms}$ ) were applied

791 every $4 \mathrm{~s}$.

792

Adenoviral knockdown. HUVECs were plated at 550,000 cell/well in 12 well plates. Cells were grown for $24 \mathrm{~h}$ in the plates and transduced with either human adenovirus type 5 with shLRRC8A (shSWELL1: Ad5-mCherry-U6-hLRRC8A-shRNA, 2.2X1010 PFU/ml, (shADV214592), Vector Biolabs), or a scrambled non-targeting control (shSCR: Ad5-U6-scramble-

$797 \mathrm{mCherry}, 1 \times 10^{10} \mathrm{PFU} / \mathrm{ml}$ ) at a multiplicity of infection (MOI) of 50 for 12 hours, and studies 798 performed 3-4 days after adenoviral transduction. The shLRRC8a targeting sequence is: GCA CAA CAT CAA GTT CGA CGT.

801 siRNA knockdown: HUVECs were plated at 360,000 cell/well in 6 well plate. Cells were grown

802 for $24 \mathrm{~h}$ (90-95\% confluency) and transduced with either a silencer select siRNA with si-

803 LRRC8a (Cat\#4392420, sense: GCAACUUCUGGUUCAAAUUTT antisense:

804 AAUUUGAACCAGAAGUUGCTG, Invitrogen) or a non-targeting control silencer select siRNA

805 (Cat\# 4390846, Invitrogen), as described previously (3). The siLRRC8a used targets a different 
806 sequence from the shRNA described above. Briefly, siRNAs were transduced twice, 24 and 72

807 hours after HUVEC plating. Each siRNA was combined with Opti-MEM (285.25 $\mu$ l, Cat\#11058-

808 021, Invitrogen) siPORT TM amine (8.75 $\mu$ l, Cat\#AM4503, Invitrogen) and the silencer select

809 siRNA $(6 \mu \mathrm{l})$ in a final volume of $300 \mu \mathrm{l}$. HUVECs were transduced over a 4-hour period at $37^{\circ} \mathrm{C}$,

810 using DMEM media $+1 \%$ FBS. After transduction, the cells were returned to media containing

811 M199, 20\% FBS, $0.05 \mathrm{~g}$ Heparin Sodium Salt, and 15 mg ECGS. Cell lysates were collected at

812 basal conditions on day 4.

Isolation of mouse lung endothelial cells

815 Isolation of mouse lung endothelial cells was performed according to the following protocol: Day

816 1- Incubate sheep anti-rat IgG Dynabeads (Invitrogen) overnight with PECAM (Sigma,

817 \#SAB4502167) and VEGFR2 (R\&D Systems, \#BAF357) antibodies at $4{ }^{\circ} \mathrm{C}$ in PBS with gentle

818 agitation. Day 2 - lungs were removed from the mice, washed in 10\% FBS/DMEM, minced into

819 1-2 $\mathrm{mm}$ squares and digested with Collagenase Type I $(2 \mathrm{mg} / \mathrm{ml}$, Gibco $)$ at $37^{\circ} \mathrm{C}$ for 1 hour with

820 agitation. The cellular digest was filtered through a $70 \mu \mathrm{m}$ cell strainer, centrifuged at $1500 \mathrm{rpm}$

821 and the cells immediately incubated with the antibody coated Dynabeads at room temperature

822 for 20 minutes. The bead-bound cells were recovered with a magnet, washed two times with

823 PBS, and plated overnight on collagen type I (100ug/ml) coated cover slips. The endothelial

824 cells were maintained in a growth media of M199, 20\% FBS, 0.05g Heparin Sodium Salt,

$82550 \mathrm{mg} / \mathrm{ml}$ ECGS, and 1x Anti-Anti.

826 Cells Culture: HUVECs were purchased from ATCC and were grown in MCDB-131- Complete

827 media overnight. HUVECs for basal condition collection were grown in growth media of M199,

$82820 \%$ FBS, 0.05g Heparin Sodium Salt (Cat\#9041-08-1, Alfa Aesar), and 15 mg ECGS (Cat\#02-

829102 , Millipore Sigma). Cells were routinely cultured on $1 \%$ of gelatin coated plates at $37^{\circ} \mathrm{C}$ at 
$8305 \% \mathrm{CO}_{2}$. For insulin stimulation (Cat\#SLBW8931), cells were serum starved for at least $13 \mathrm{~h}$ in

831 1\% FBS (Atlanta Bio selected, Cat \#S11110) or without FBS using endothelial cells growth

832 basal medium (Lonza cat\#cc-3121) instead of MCDB-131- complete media. Insulin stimulation

833 was used for the times indicated at $100 \mathrm{nM}$.

834 Immunoblotting: Cells were harvested in ice-cold lysis buffer (150 mM NaCl, 20 mM HEPES,

$8351 \%$ NP-40, 5mM EDTA, pH 7.5) with added proteinase/phosphatase inhibitor (Roche). Cells

836 were kept on ice with gentle agitation for 20 minutes to allow complete lysis. Lysate scraped into

$8371.5 \mathrm{ml}$ tubes and cleared of debris by centrifugation at $14,000 \times \mathrm{g}$ for 20 minutes at $4{ }^{\circ} \mathrm{C}$.

838 Supernatant were transferred to fresh tube and solubilized protein was measured using a DC

839 protein assay kit (Bio-Rad). For immunoblotting an appropriate volume of $1 \mathrm{x}$ Laemmli (Bio-rad)

840 sample loading buffer was added to the sample (10 $\mu \mathrm{g}$ of protein), which then heated at $90^{\circ} \mathrm{C}$

841 for 5 min before loading onto 4-20\% gel (Bio-Rad). Proteins were separated using running

842 buffer (Bio-Rad) for $2 \mathrm{~h}$ at 150 V. Proteins were transferred to PVDF membrane (Bio-Rad) and

843 membrane blocked in $5 \%(w / v)$ BSA in TBST or $5 \%(w / v)$ milk in TBST at room temperature for

8442 hours. Blots were incubated with primary antibodies at $4{ }^{\circ} \mathrm{C}$ overnight, followed by secondary

845 antibody (Bio-Rad, Goat-anti-mouse \#170-5047, Goat-anti-rabbit \#170-6515, all used at

846 1:10000) at room temperature for one hour. Membranes were washed3 times and incubated in

847 enhanced substrate Clarity (Bio-Rad) and imaged using a ChemiDoc XRS using Image Lab

848 (Bio-Rad) for imaging and analyzing protein band intensities. ß-Actin or GAPDH levels were

849 quantified to correct for protein loading.

850 Immunoprecipitation: Cells were seeded on gelatin-coated $10 \mathrm{~cm}$ dishes in complete media

851 for 24 h. Adenoviruses, Ad5-mCherry-U6-hLRRC8A-shRNA or Ad5-U6-scramble-mCherry were

852 added to cells for $12 \mathrm{~h}$. After 4 days cells were serum starved for $16 \mathrm{~h}$ with basal media contain

$8531 \%$ serum before stimulation with insulin $(10 \mathrm{nM} / \mathrm{ml})$. Cells were harvested in ice-cold lysis

854 buffer (150 mM NaCl, 20 mM HEPES, 1\% NP-40, 5mM EDTA, pH 7.5) with added 
855 proteinase/phosphatase inhibitor (Roche) and kept on ice with gentle agitation for 15 minutes to

856 allow complete lysis. Lysated were incubated with anti-Grb2 antibody $(20 \mu \mathrm{g} / \mathrm{ml})$ or control rabbit

$857 \operatorname{lgG}\left(20 \mu \mathrm{g} / \mathrm{ml}\right.$, Santa Cruz sc-2027) rotating end over end overnight at $4{ }^{\circ} \mathrm{C}$. Protein $\mathrm{G}$

858 sepharose beads (GE) were added to this for a further $4 \mathrm{~h}$ before samples were centrifuged at

$85910,000 \times \mathrm{g}$ for 3 minutes and washed three times with RIPA buffer and re-suspended in laemmli

860 buffer (Bio-Rad), boiled for 5 minutes, separated by SDS-PAGE gel followed by the western blot

861 protocol.

862

863 Stretch assay: Equal amounts of cells were plated in each well in 6 well plated BioFlex coated with Laminin (BF-3001CCase) culture plate and seeded to approximately $90 \%$ confluence.

Plates were placed into a Flexcell Jr. Tension System (FX-6000T), and incubated at $37^{\circ} \mathrm{C}$ with lysed and protein isolated for subsequent Western blots.

Immunofluorescence imaging: Cells were plated on gelatin-coated glass coverslips. Cells on

871 cover slip were washed in PBS and fixed with 2\% (w/v) PFA for 20 minutes at room

872 temperature. PFA were washed three times with PBS and permeabilized in PBS containing 0.2

$873 \%$ Triton X-100 for 5 minutes at room temperature. Cells on coverslips were washed in PBS and

874 blocked for 30 minutes at room temperature with TBS containing $0.1 \%$ Tween-20 and $5 \%$ BSA.

875 Cells on coverslip were incubated overnight at $4^{\circ} \mathrm{C}$ with primary antibody $(1: 250)$ in TBS

876 containing $0.1 \%$ Tween-20 and $1 \%$ BSA. Cells were then washed in PBS 5x and incubated for 2

877 hours at room temperature with 5\% BSA in TBST. Cells were washed three times in TBST and

878 then incubated with secondary antibodies at 1:1000 dilution (Invitrogen, Anti-Rabbit 488,

879 A11070; Anti-mouse 568, A11019; Anti-mouse 488, A11017) for $1 \mathrm{~h}$ at room temperature. 
880 Coverslips were then incubated for 10 minutes with Topro 3 (T3605, Thermofisher) or mounted

881 with mounting media containing DAPI (Invitrogen), to visualize nuclei. Images were taken using

882 Axiocam 503 Mono Camera controlled by Zeiss Blue using a Plan-Apochromate 40x oil

883 immersion objective.

885 Ex vivo sprouting angiogenesis assay. Following Avertin injection and cervical dislocation, penicillin and streptomycin. Using iris scissors, the aorta was cut into aortic rings of 1 2 $\mathrm{mm}$ cross sectional slices. $50 \mu \mathrm{l}$ of Matrigel was used to coat the center of coverslips in 24 well plates for two hours at $37^{\circ} \mathrm{C}$ in the incubator to solidify the Matrigel. Aorta rings were then seeded and transplanted on Matrigel (BD Biosciences, Cat\#356231) on coverslips. After seeding the aortic rings, plates were incubated in $37^{\circ} \mathrm{C}$ without medium for 10 minutes to allow the ring to attach to the Matrigel. Complete medium was added to each well and incubated at $37^{\circ} \mathrm{C}$ with $5 \% \mathrm{CO}_{2}$ for $48-72 \mathrm{~h}$. Phase contrast photos of individual explants were taken using a 10x/0.75 NA objective Olympus IX73 microscope (Olympus, Japan) fitted with camera (Orca flash 4.0+, Hamamatsu, Japan). The areas of sprouting, number of tips cells and length of the 896 tube for each condition were quantified with computer software ImageJ 1.52i (National Institute 897 of Health). Cells were incubated with SWELL1 (1:250) and CD31 (1:250) primary antibodies in $898 \quad 0.1 \%$ Tween-20 and $1 \%$ BSA overnight, and then incubated with secondary antibodies 899 (Invitrogen, Anti-Rabbit 488, A11070; Anti-Mouse 568, A11019). Cells were then incubated for 90010 minutes with Topro 3 (T3605, Thermofisher) at room temperature.

902 Retina imaging. Ketamine (Akorn Animal Health, $100 \mathrm{mg} / \mathrm{ml}$ ) was prepared and mixed with 903 Xylazine then stored at room temperature. Animals were anesthetized with 87.5/12.mg/kg BW 904 via intraperitoneal (IP) injection. Eyes were topically anesthetized with proparacaine and dilated 905 with tropicamide. Fluorescein $(100 \mathrm{mg} / \mathrm{ml}$, Akorn $\mathrm{Inc}$ ) diluted with sterile saline was administered 
by IP injection $(50 \mu \mathrm{l})$, mice positioned on Micron imaging platform. Images of the eyes were

907 taken from the start of fluorescein infusion with the Micron camera with a 450-650 nm excitation

908 filter and 469-488 nm barrier filter at 30 frame/sec using Micron software for 30 seconds. Data

909 were converted into tiff image for further analysis.

910

911 Angiotensin-II infusion: Infusion studies carried out using Azlet osmotic minipumps (Model

912 1004). Angiotensin-II (BACHEM) dissolved in saline was filled in the minipumps and were

913 prepared to maintain infusion rate of $600 \mathrm{ng} / \mathrm{kg} / \mathrm{min}$ for four weeks. The mice were anesthetized

914 under $2 \%$ isofluorane and the minipumps were implanted subcutaneously on the dorsal aspect

915 of the mice.

\section{Blood pressure recordings}

919 Systolic tail-cuff blood pressure (BP) measurements were carried out using computerized tail-

920 cuff system BP-2000 (Visitech Systems) at the same time of day. Mice were first acclimated to

921 the device by performing 3 days of measurements (20 sequential measurements/day) and then

922 mean blood pressure readings were obtained by averaging 3-5 days of measurements (not

923 inclusive of the 3 acclimation days).

$925 \quad$ RNA sequencing

926 RNA quality was assessed by Agilent BioAnalyzer 2100 by the University of lowa Institute of

927 Human Genetics, Genomics Division. RNA integrity numbers greater than 8 were accepted for

928 RNAseq library preparation. RNA libraries of 150 bp PolyA-enriched RNA were generated, and

929 sequencing was performed on a HiSeq 4000 genome sequencing platform (Illumina).

930 Sequencing results were uploaded and analyzed with BaseSpace (Illumina). Sequences were

931 trimmed to $125 \mathrm{bp}$ using FASTQ Toolkit (Version 2.2.0) and aligned to Mus musculus mmp10 
932 genome using RNA-Seq Alignment (Version 1.1.0). Transcripts were assembled and differential

933 gene expression was determined using Cufflinks Assembly and DE (Version 2.1.0). Ingenuity

934 Pathway Analysis (QIAGEN) was used to analyze significantly regulated genes which were

935 filtered using cutoffs of $>1.5$ fragments per kilobase per million reads, $>1.5$ fold changes in gene

936 expression, and a false discovery rate of $<0.05$. Heatmaps were generated to visualize

937 significantly regulated genes. Data have been deposited in GEO (accession\# TBD).

938

\section{Metabolic phenotyping}

940 Mice were fasted for $6 \mathrm{~h}$ prior to glucose tolerance tests (GTT). Baseline glucose levels at 0 min

941 timepoint (fasting glucose, FG) were measured from blood sample collected from tail snipping

942 using glucometer (Bayer Healthcare LLC). $0.75 \mathrm{~g} \mathrm{D}-$ Glucose/kg body weight were injected (i.p.)

943 for HFHS mice, respectively and glucose levels were measured at 7, 15, 30, 60, 90 and 120 min

944 timepoints after injection. For insulin tolerance tests (ITTs), the mice were fasted for $4 \mathrm{~h}$. Similar

945 to GTTs, the baseline blood glucose levels were measured at 0 min timepoint and 15, 30, 60, 90

946 and 120 min timepoints post-injection (i.p.) of insulin (HumulinR, $1.25 \mathrm{U} / \mathrm{kg}$ body weight).

\section{References}

949

950

951

952

953

954

955

956

957

958

959

1. Kang C, Xie L, Gunasekar SK, Mishra A, Zhang Y, Pai S, et al. SWELL1 is a glucose sensor regulating beta-cell excitability and systemic glycaemia. Nat Commun. 2018;9(1):367.

2. Zhang Y, Xie L, Gunasekar SK, Tong D, Mishra A, Gibson WJ, et al. SWELL1 is a regulator of adipocyte size, insulin signalling and glucose homeostasis. Nature cell biology. 2017;19(5):504-17.

3. Koh W, Stratman AN, Sacharidou A, and Davis GE. In vitro three dimensional collagen matrix models of endothelial lumen formation during vasculogenesis and angiogenesis. Methods Enzymol. 2008;443:83-101.

960

961 
A

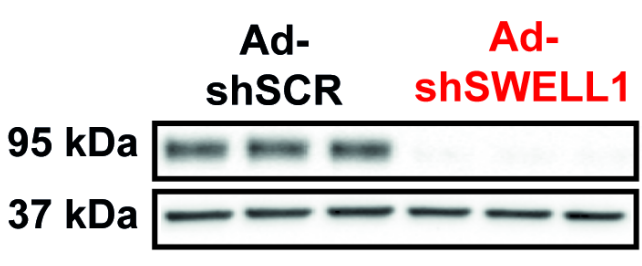

IB:SWELL1 IB:GAPDH

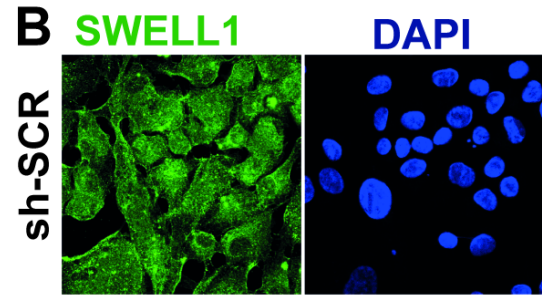

Merged
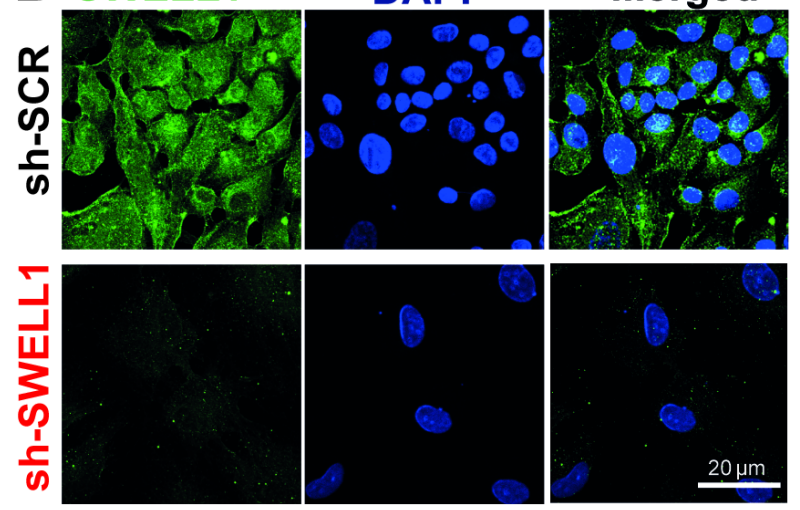

C

D
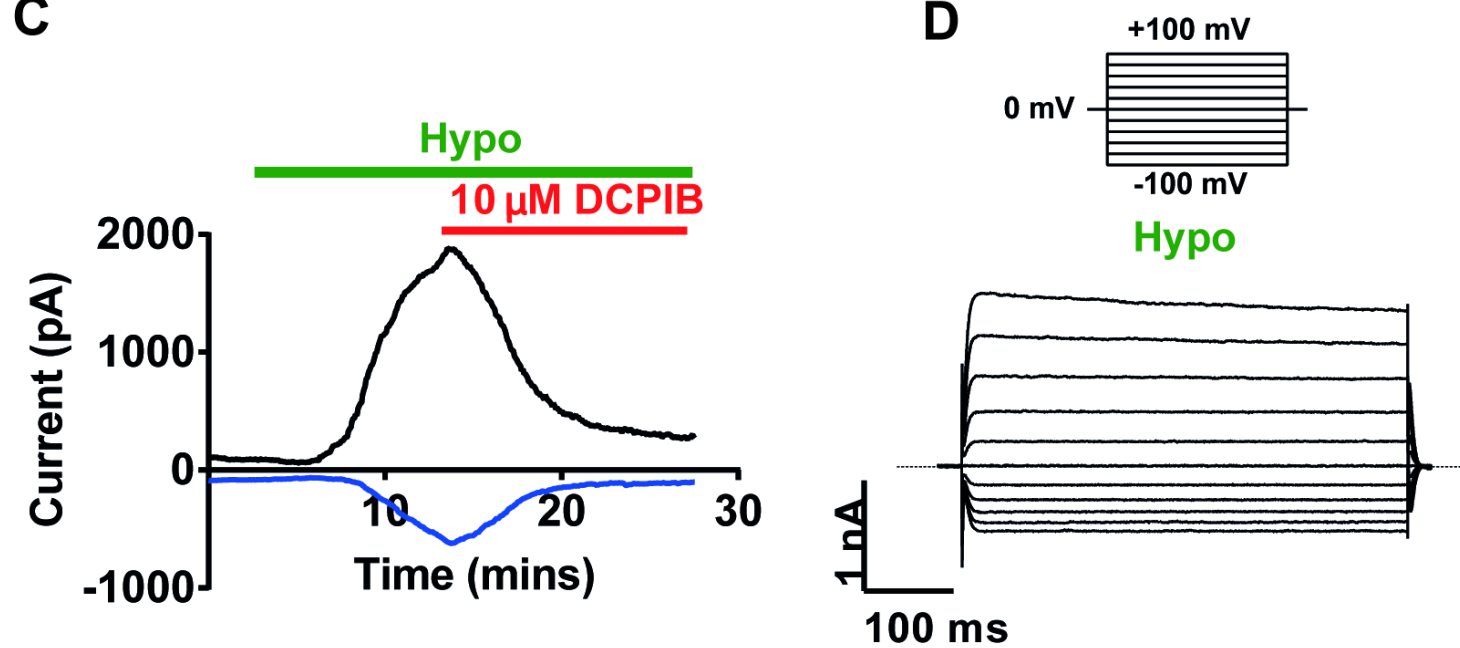

$10 \mu M$ DCPIB
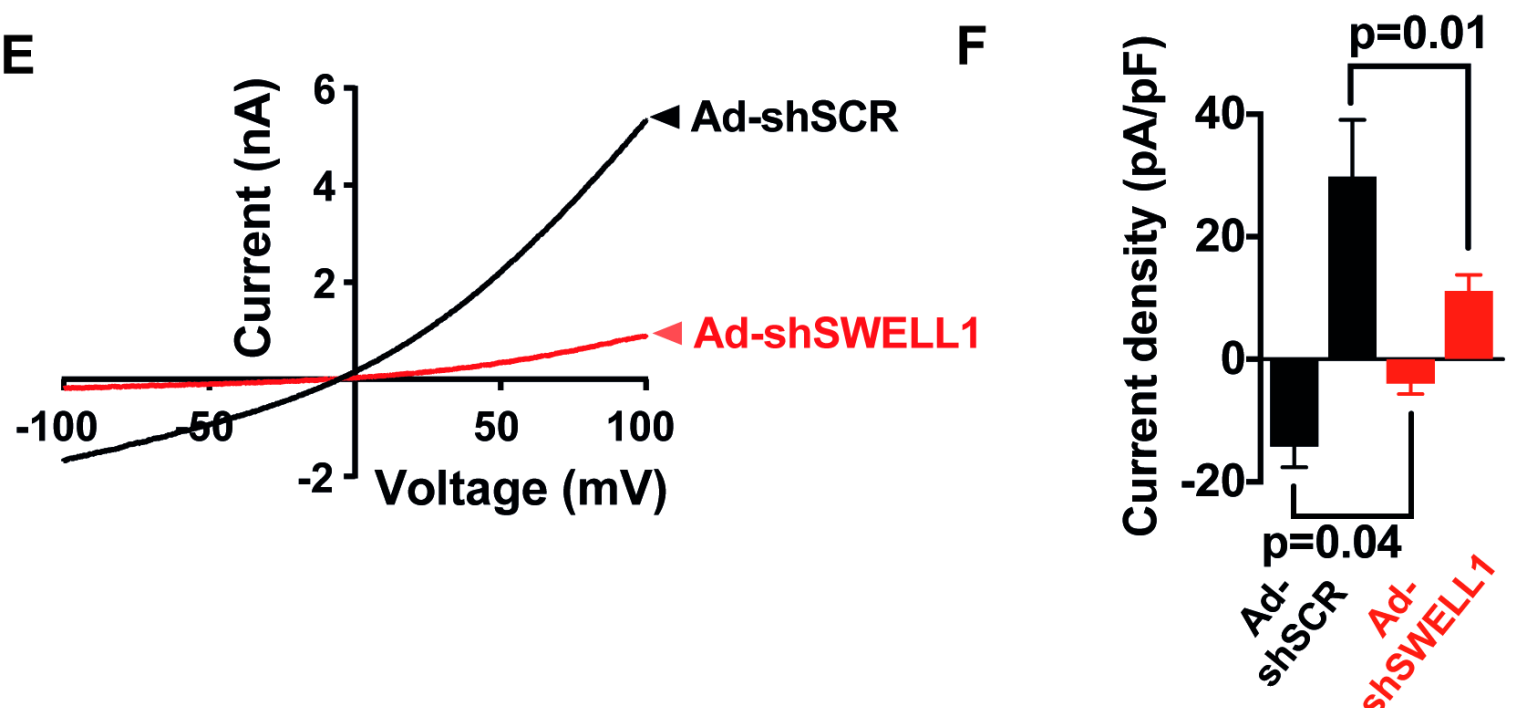

Figure 1. SWELL1 mediates VRAC currents in human umbilical vein endothelial cells (HUVECs). 
A

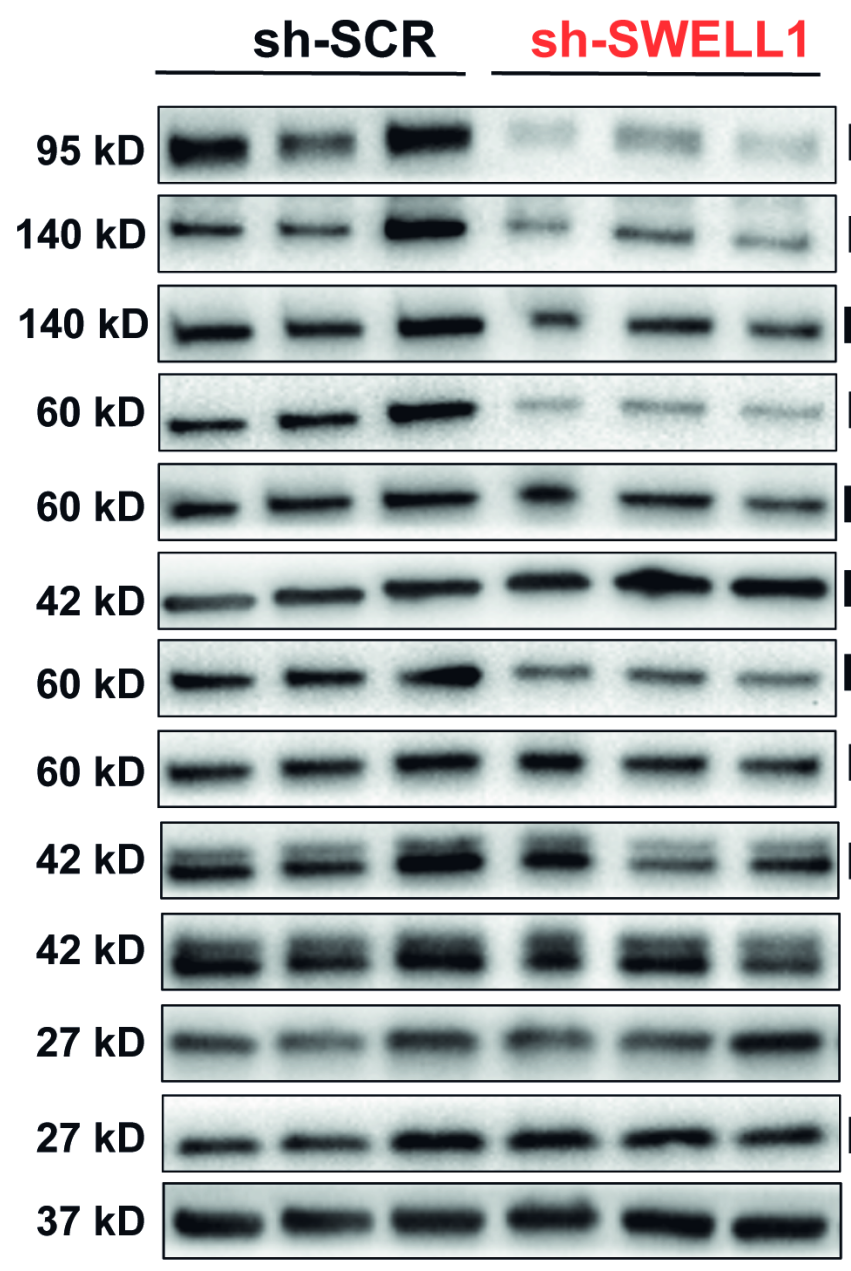

IB: SWELL1

IB: $p$-eNOS

IB: eNOS

IB: pAkt2

IB: Total Akt2

IB: $\beta$-Actin

IB: pAkt1

IB: Total Akt1

IB: pErk1/2

IB: Total Erk1/2

IB: pS6K

IB: Total S6K

IB: GAPDH
B SWELL1

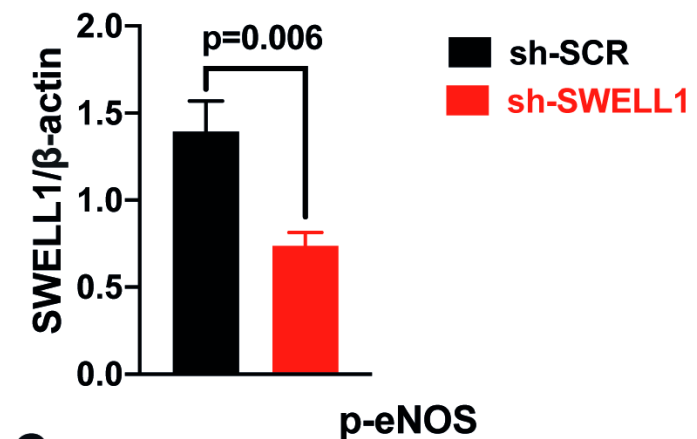

C
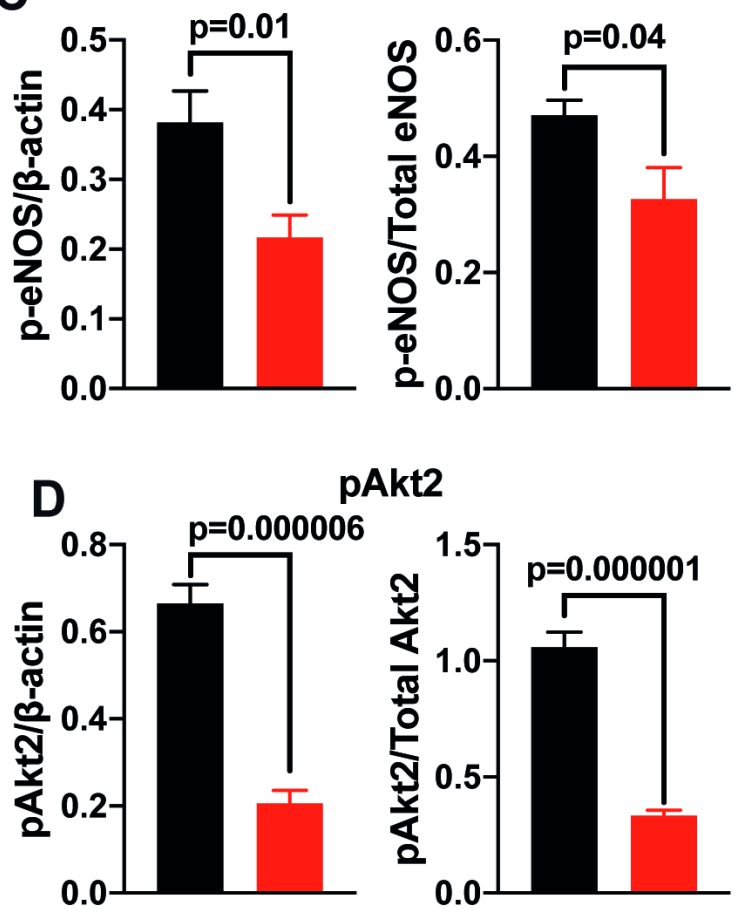

pAkt2

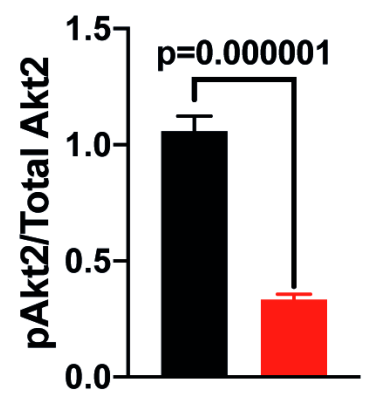

E
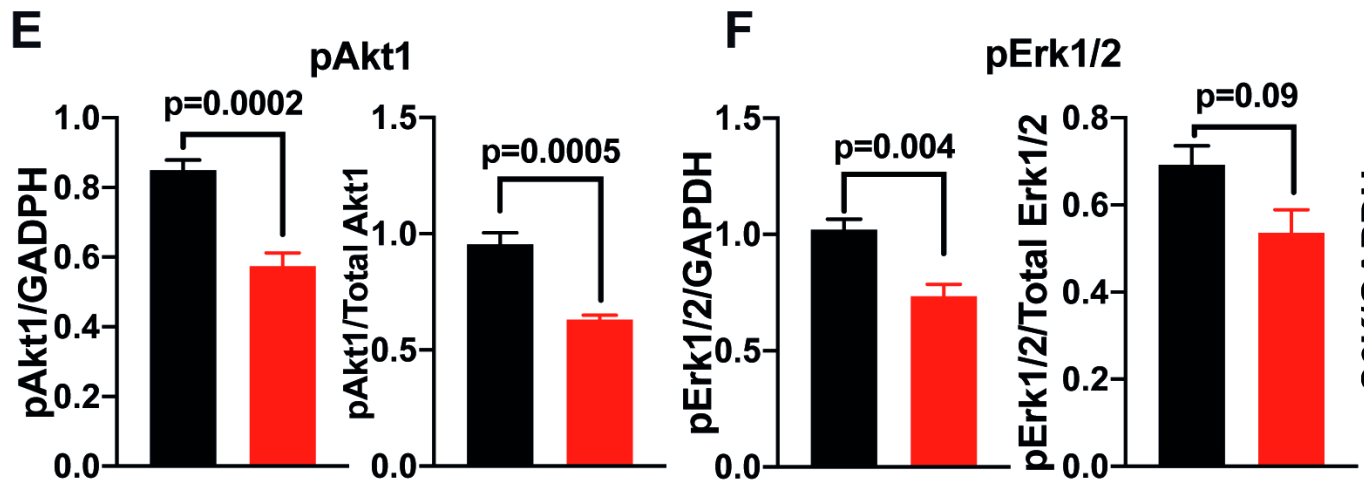
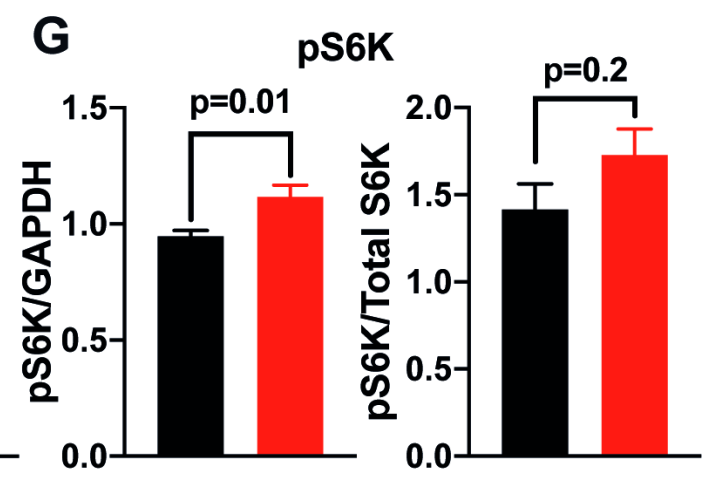

Figure 2. SWELL1 regulates PI3K-AKT-eNOS, ERK and mTOR signaling in endothelium. 
A

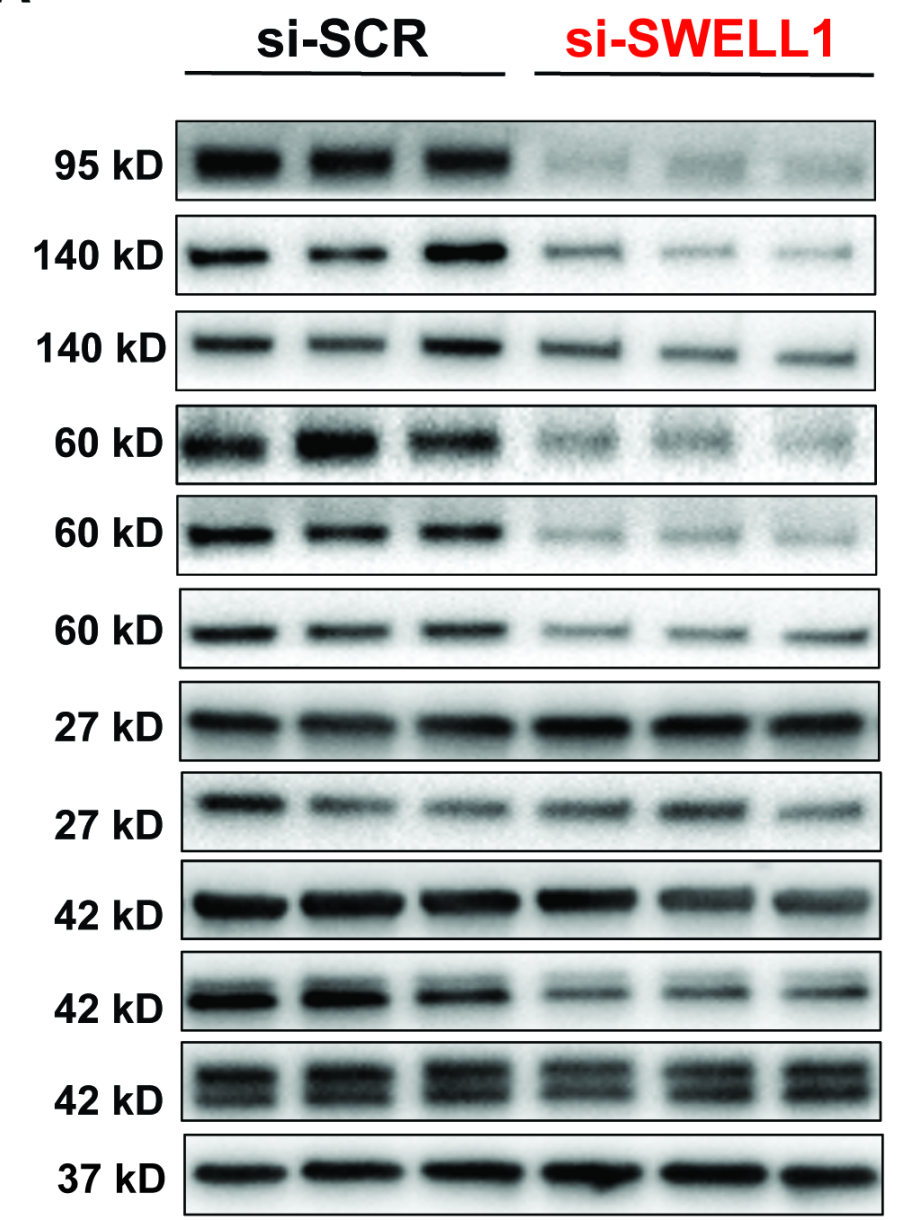

IB: SWELL1

IB: p-eNOS

IB: eNOS

IB: pAkt2

IB: pAkt1

IB: Akt

IB: pS6K

IB: Total S6K

IB: $\boldsymbol{\beta}$-Actin

IB: pErk1/2

IB: Total Erk1/2

IB: GAPDH

pAkt1

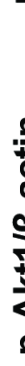

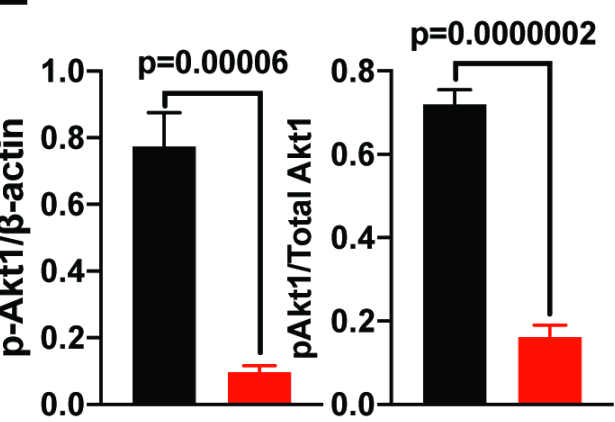

F

pErk1/2

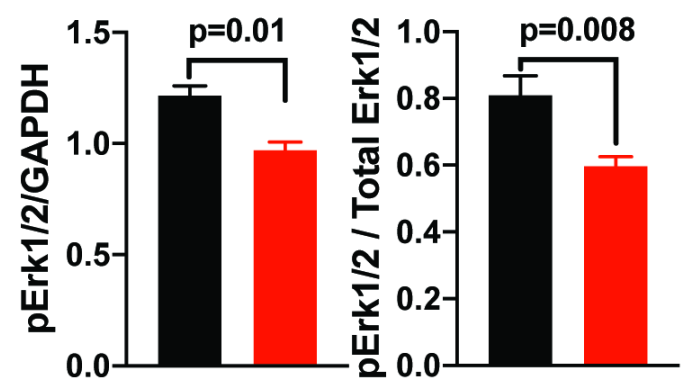

B SWELL1

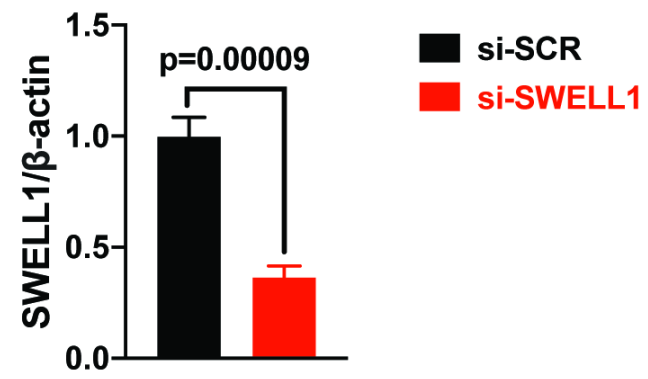

C

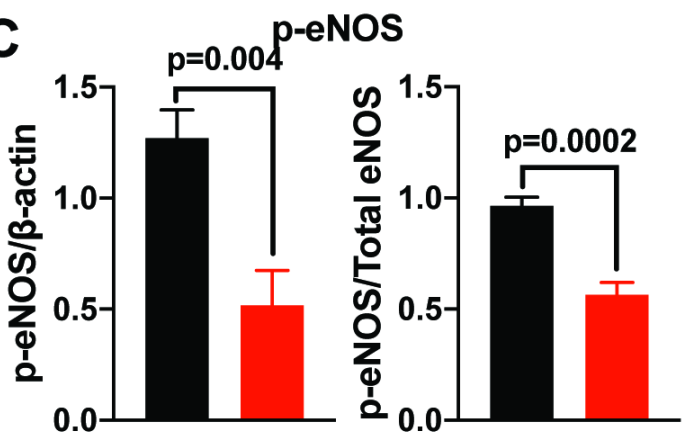

D
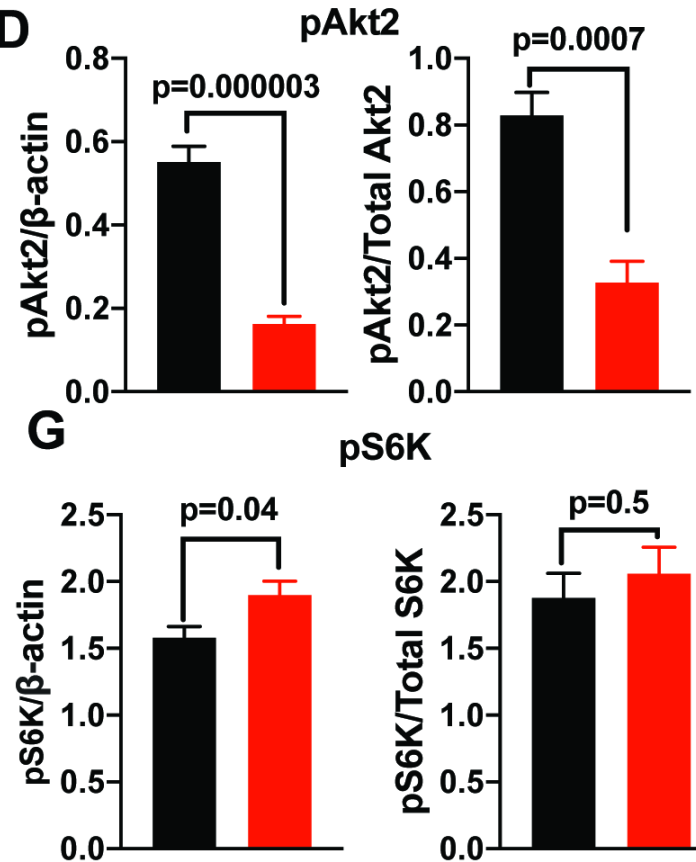

pS6K

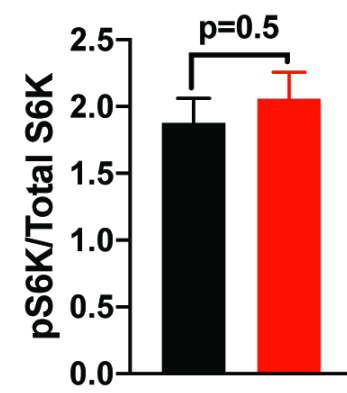

Figure 2- Figure Supplement 1. SWELL1 regulates PI3K-AKT-eNOS, ERK and mTOR signaling in endothelium. 
bioRxiv preprint doi: https://doi.org/10.1101/2020.08.04.236182; this versign posted August 4, 2020. The copyright holder for this preprint

A (which was not certified by peer review) is the author/funder, who has grant 6 bioRxiv a license to display the preprint in perpetuity. It is made available under aCC-BY 4.0 International license. $\mathbf{I P : G r b 2}$

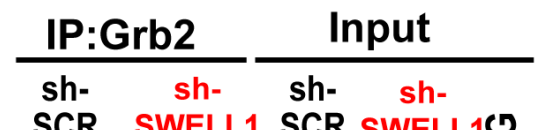

Insulin: $\frac{\text { SCR }}{-+} \frac{\text { SWELL1 }}{-+} \frac{\text { SCR }}{-+} \frac{\text { SWELL10 }}{-+}$

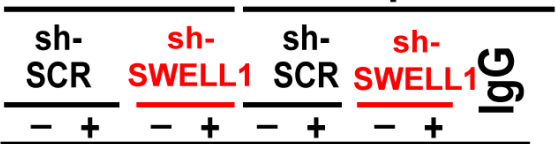

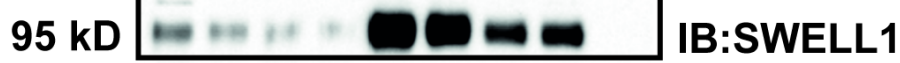

$21 \mathrm{kD}$ -

IB:Cav1

25 kD $-\infty-\ldots$ IB:Grb2

$37 \mathrm{kD}$

- -1

IB:GAPDH

SWELL1/Grb2: Ț

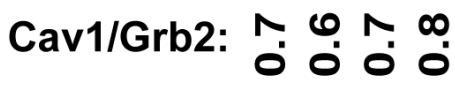

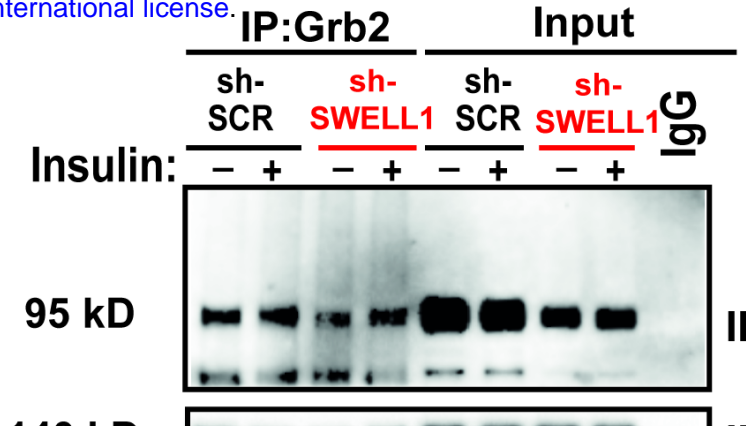

IB:SWELL1

140 kD $----\infty 6 \infty$

IB:Total eNOS

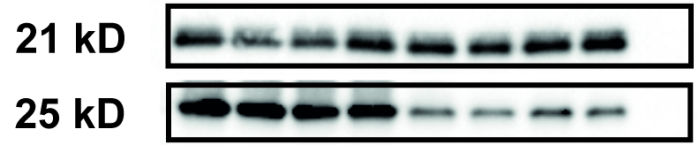

IB:Cav1

37 kD

$-\infty$

IB:Grb2

IB:GAPDH

Total eNOS/Grb2: 帒

C
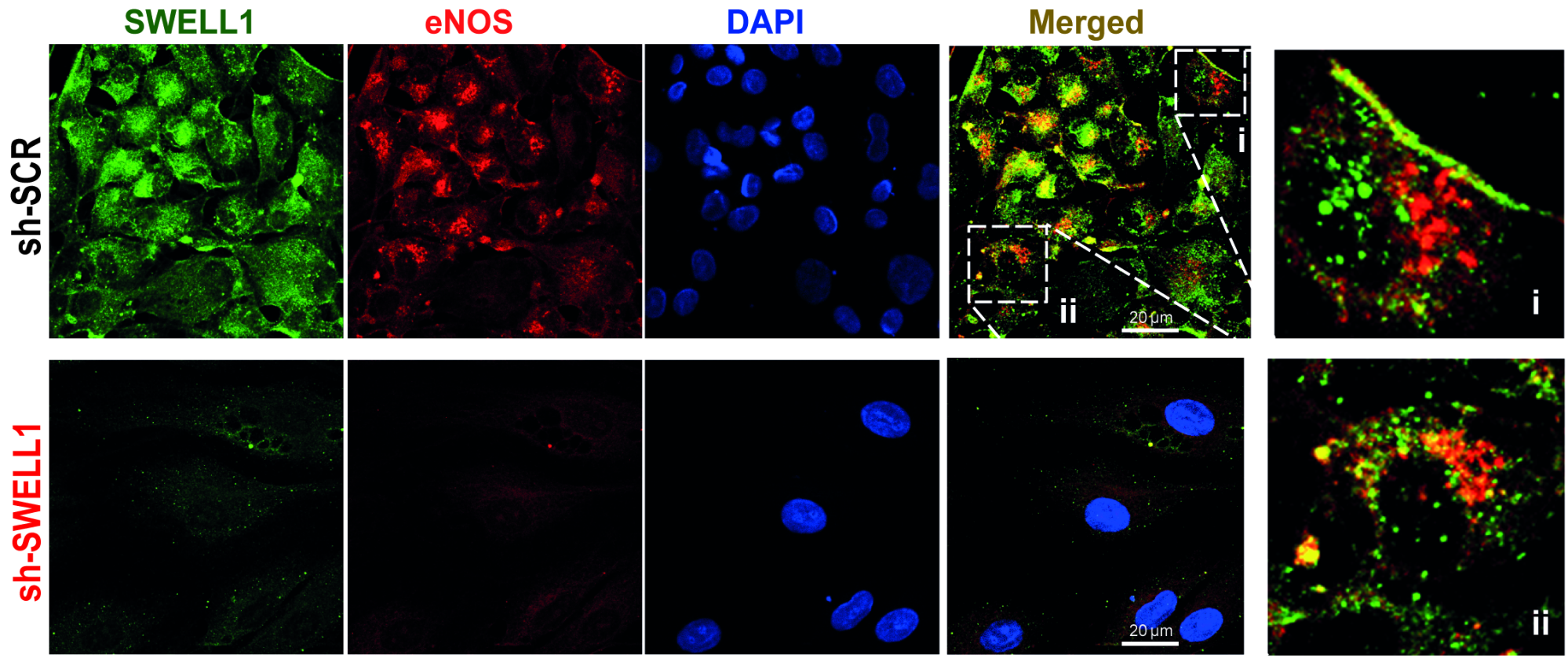

D

E

SWELL1 expression eNOS expression
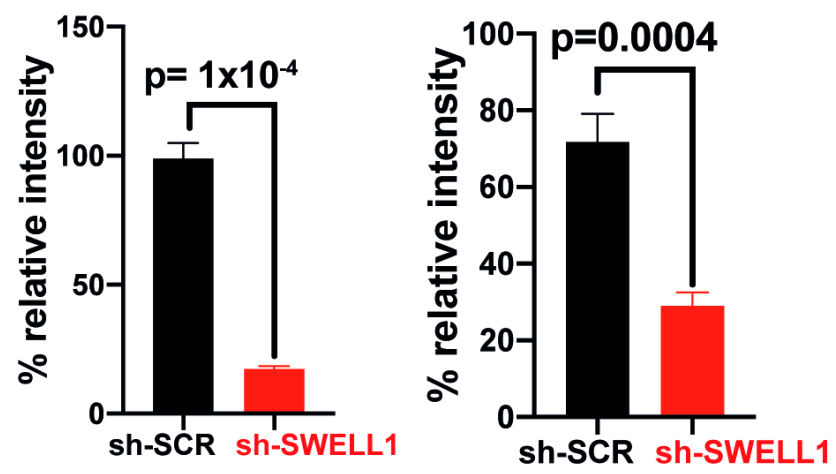

Figure 3. SWELL1 interacts with Grb2, Cav1 and eNOS in human endothelium. 


\section{sh-SCR}

\section{SWELL1}

1.9.

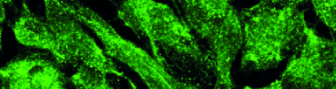

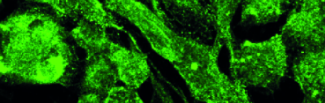

(3)

(3) $+1,4$

(5)

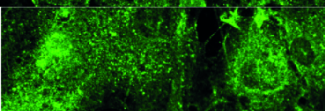

(3)

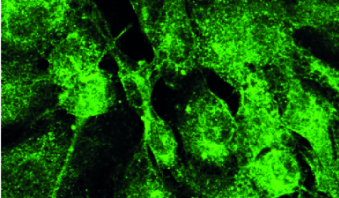

$\left(v_{1}, 5,4\right)$

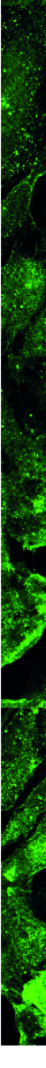

eNOS

Merge

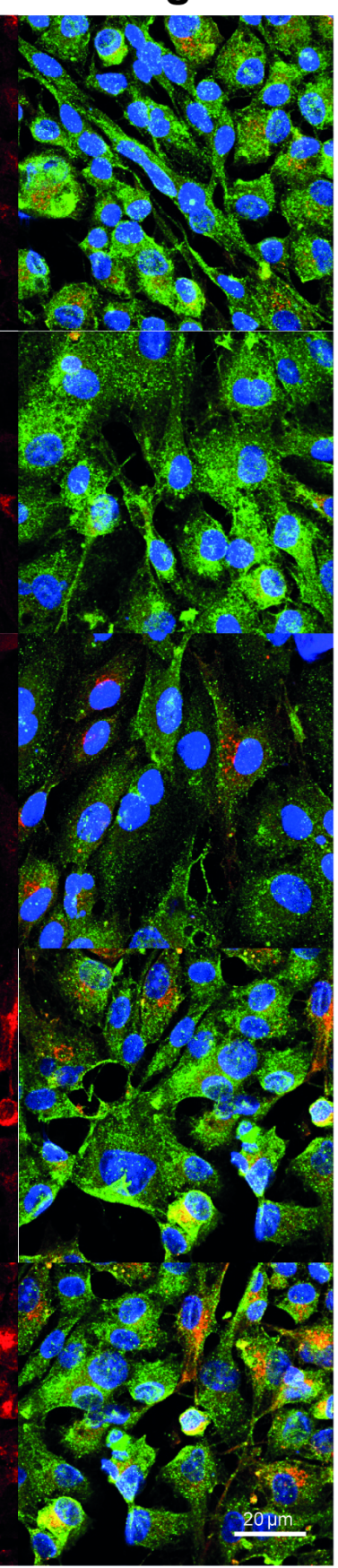

\section{Sh-SWELL1}

SWELL1 eNOS

Merge

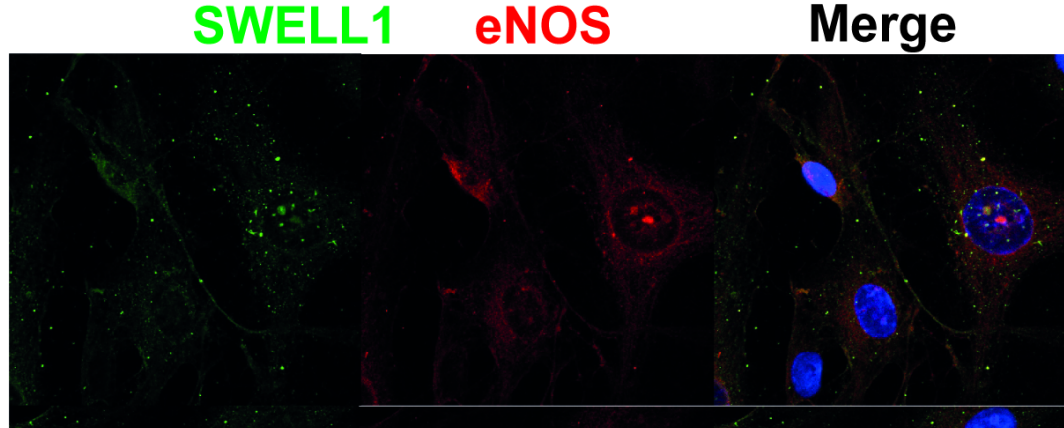


bioRxiv preprint doi: https://doi.org/10.1101/2020.08.04.236182; this version posted August 4, 2020. The copyright holder for this preprint (which was not certified by peer review) is the author/funder, who has granted bioRxiv a license to display the preprint in perpetuity. It is made
A $\quad 30$ min Stretching

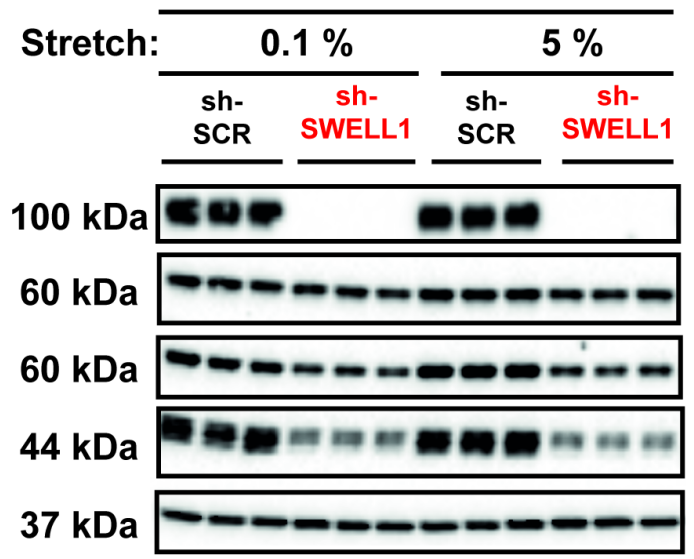
IB:SWELL1
IB:pAkt1
IB:pAkt2
IB:pErk1/2
IB:GAPDH

B

pAkt1/GAPDH
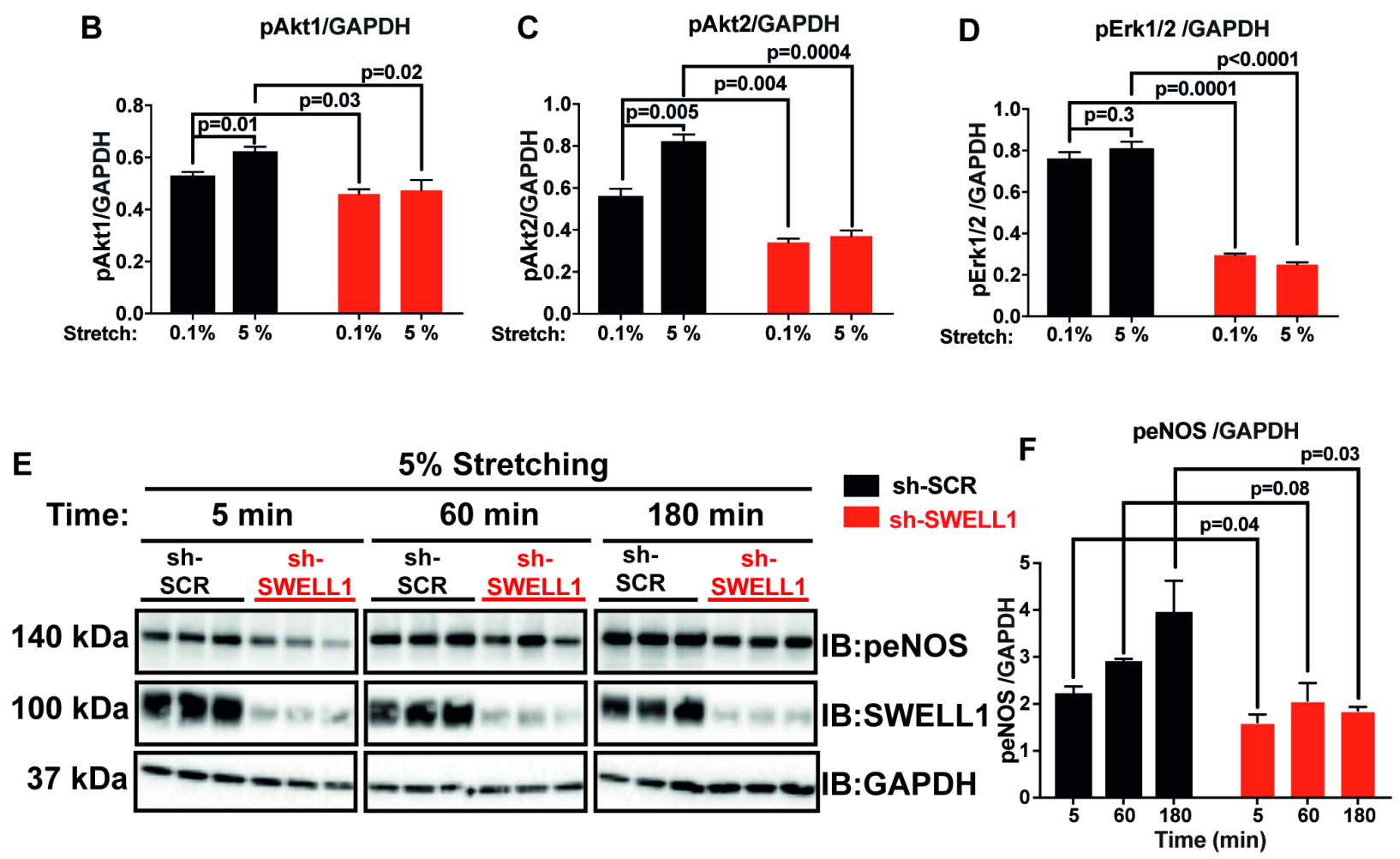

Figure 4. SWELL1 is required for intact stretch-induced AKT-eNOS signaling. 
A B
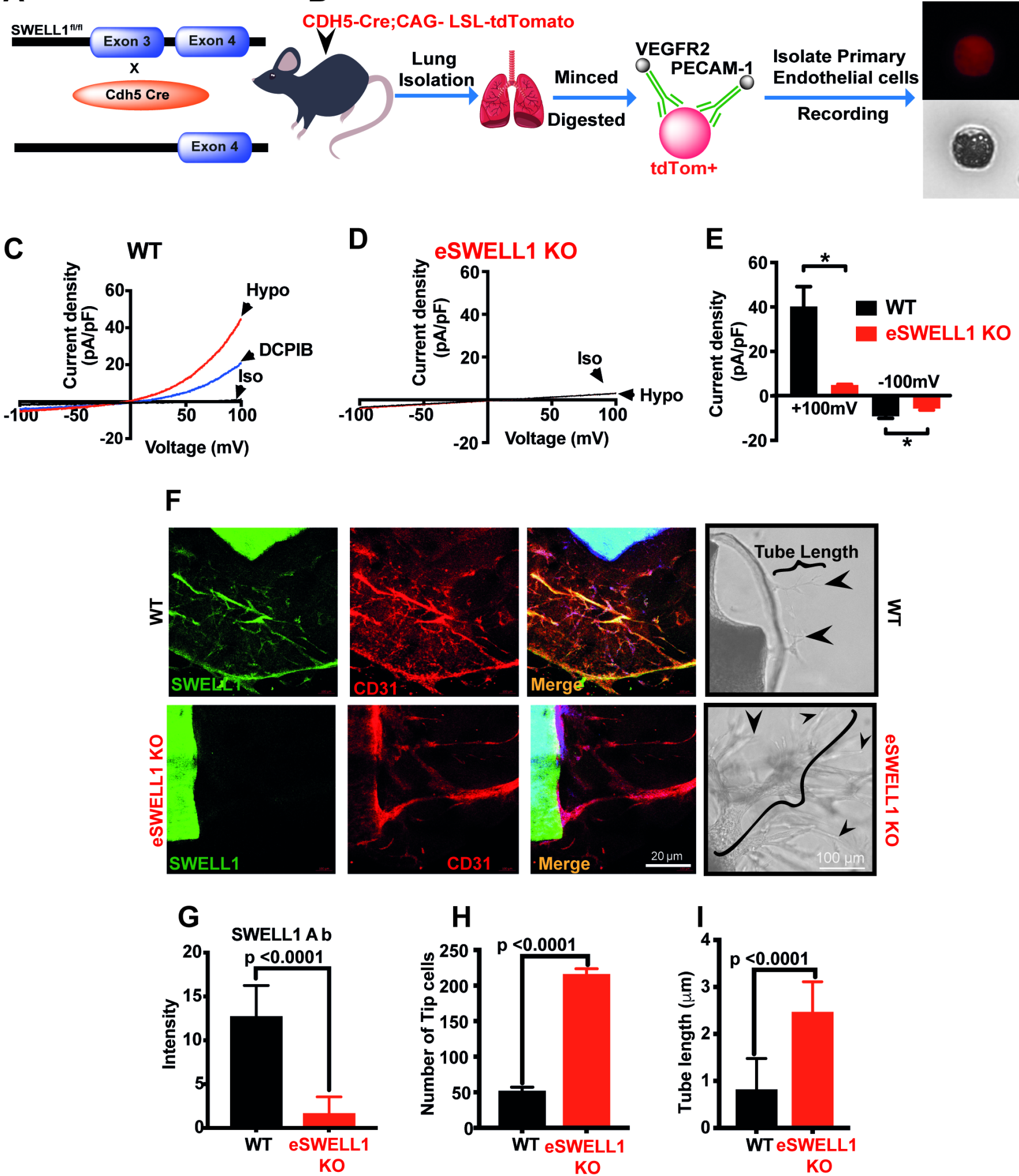

Figure 5. Endothelium-specific SWELL1 KO mice exhibit enhanced tube formation from aortic explants. 

available under aCC-BY 4.0 International license.

A

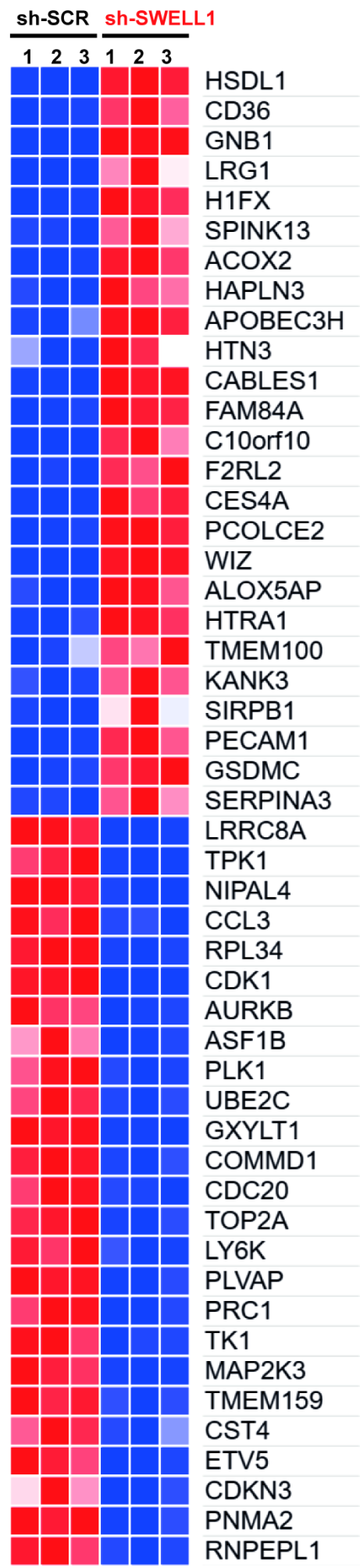

B

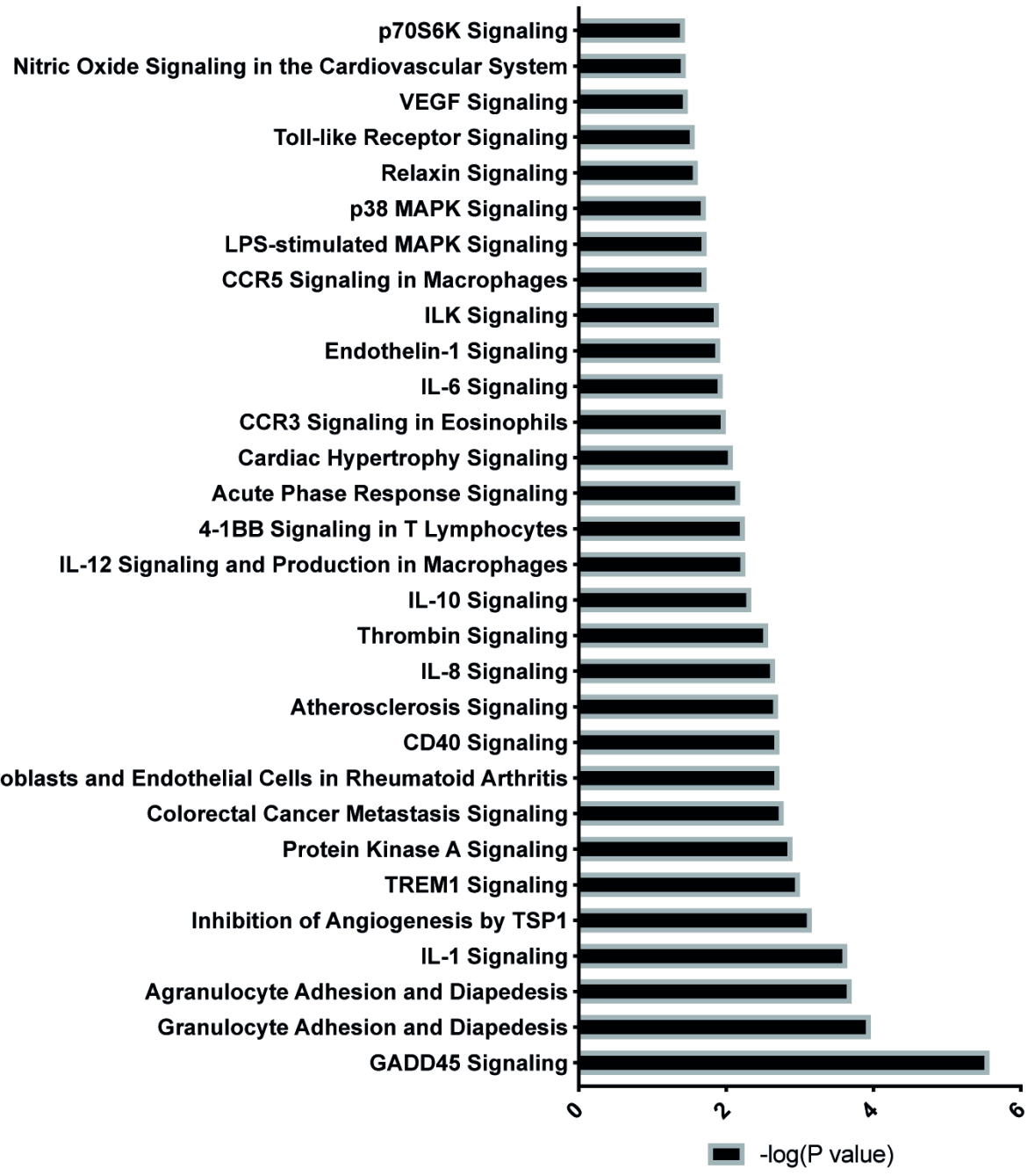
Colorectal Cancer Metastasis Signaling 
A

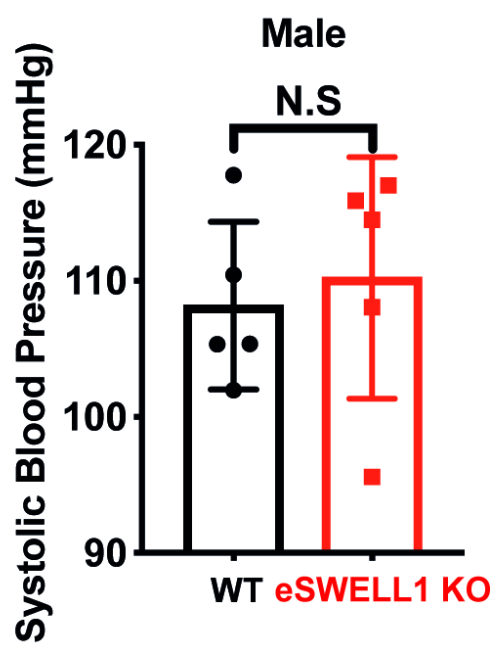

B

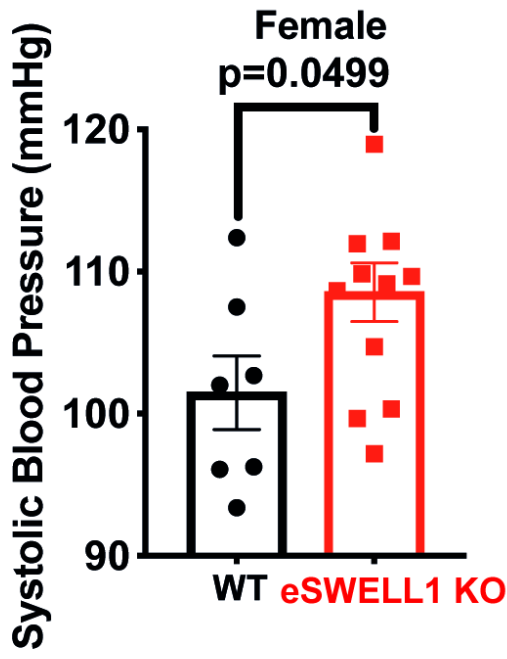

C

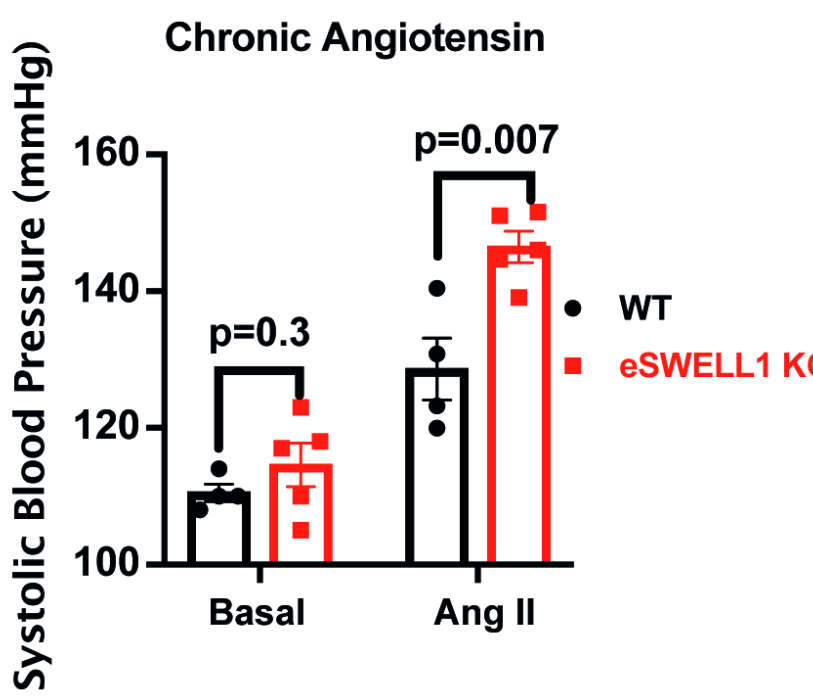

Figure 7. Endothelial-targeted SWELL1 deletion predisposes to systolic hypertension. 


\section{A}

$\xi$
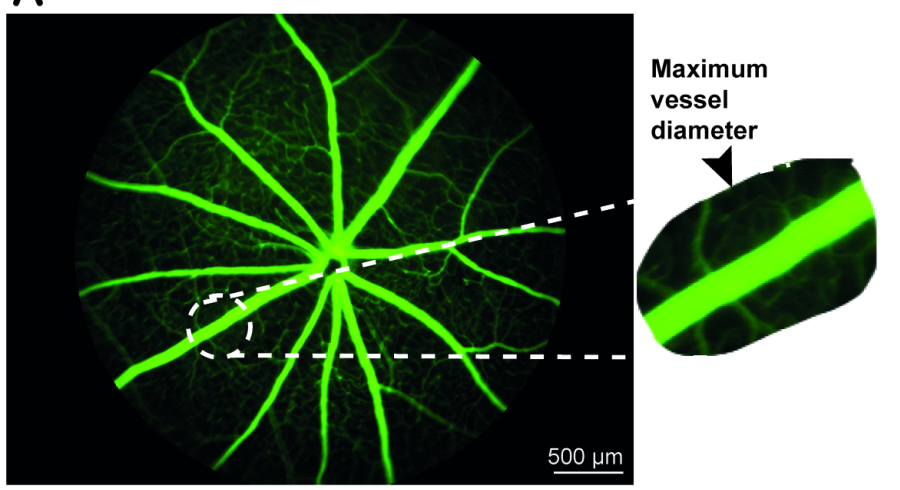

B

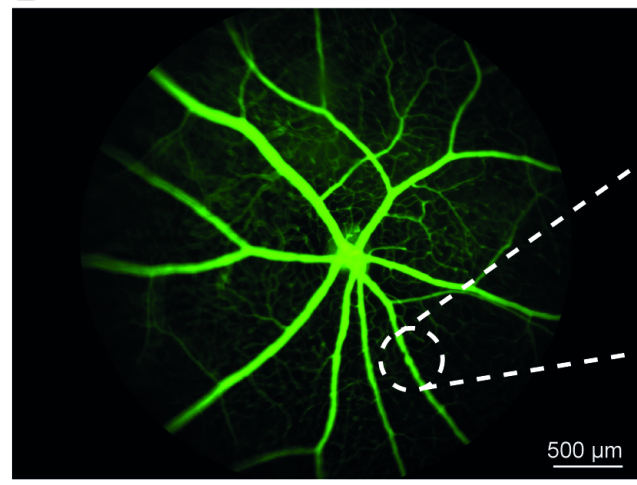

Minimum

vessel

diameter
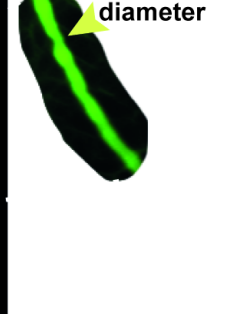

$\mathbf{F}$
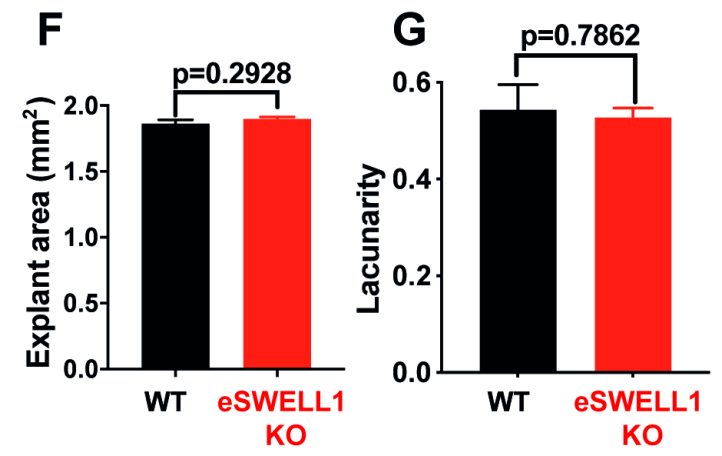

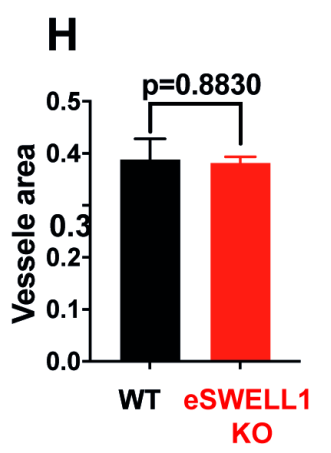

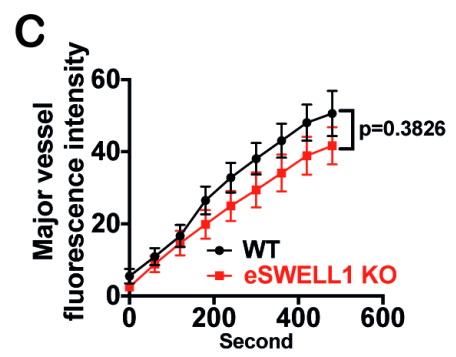
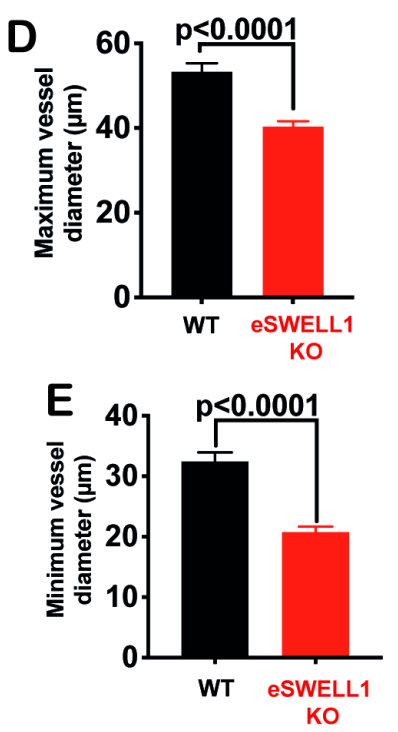

Figure 8- Figure Supplement-1. Endothelium-specific SWELL1 KO mice exhibit mild retinal microvascular disease at baseline. 
A
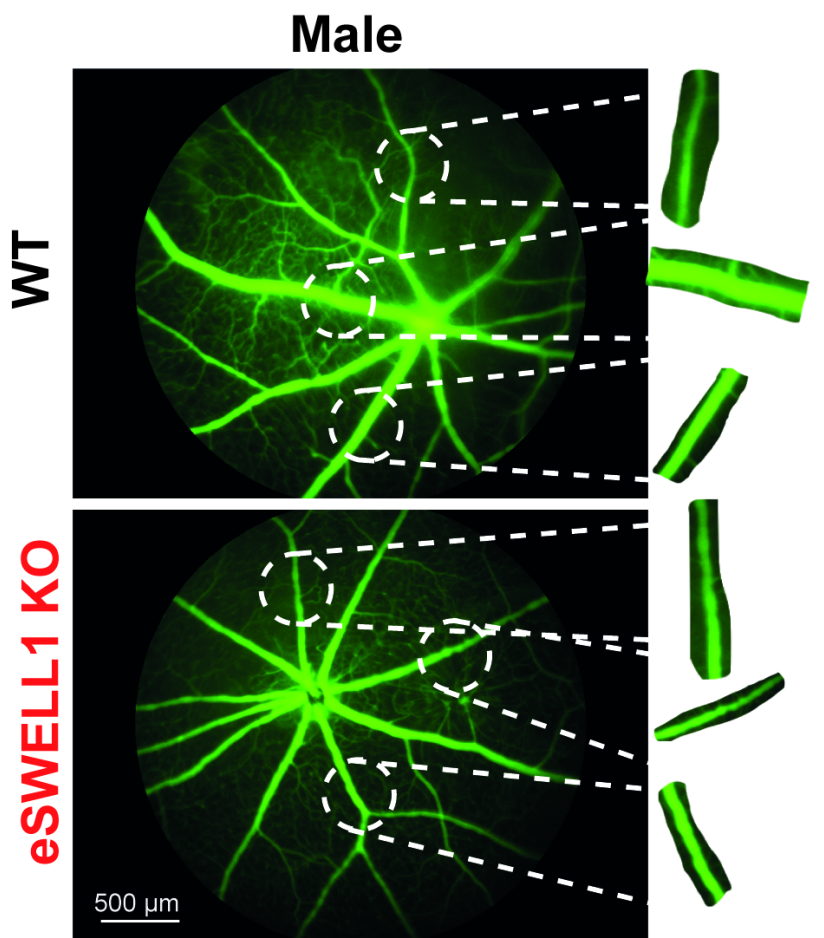

B

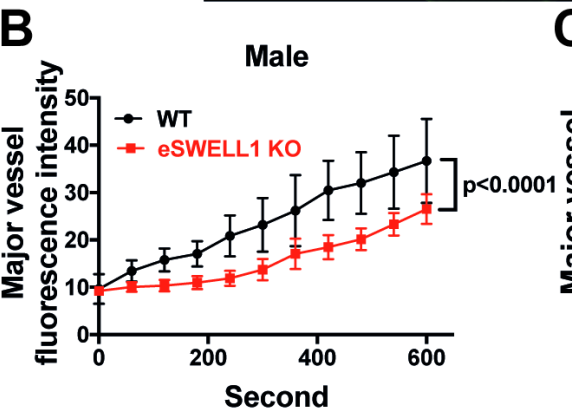

E

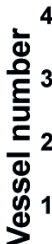

.
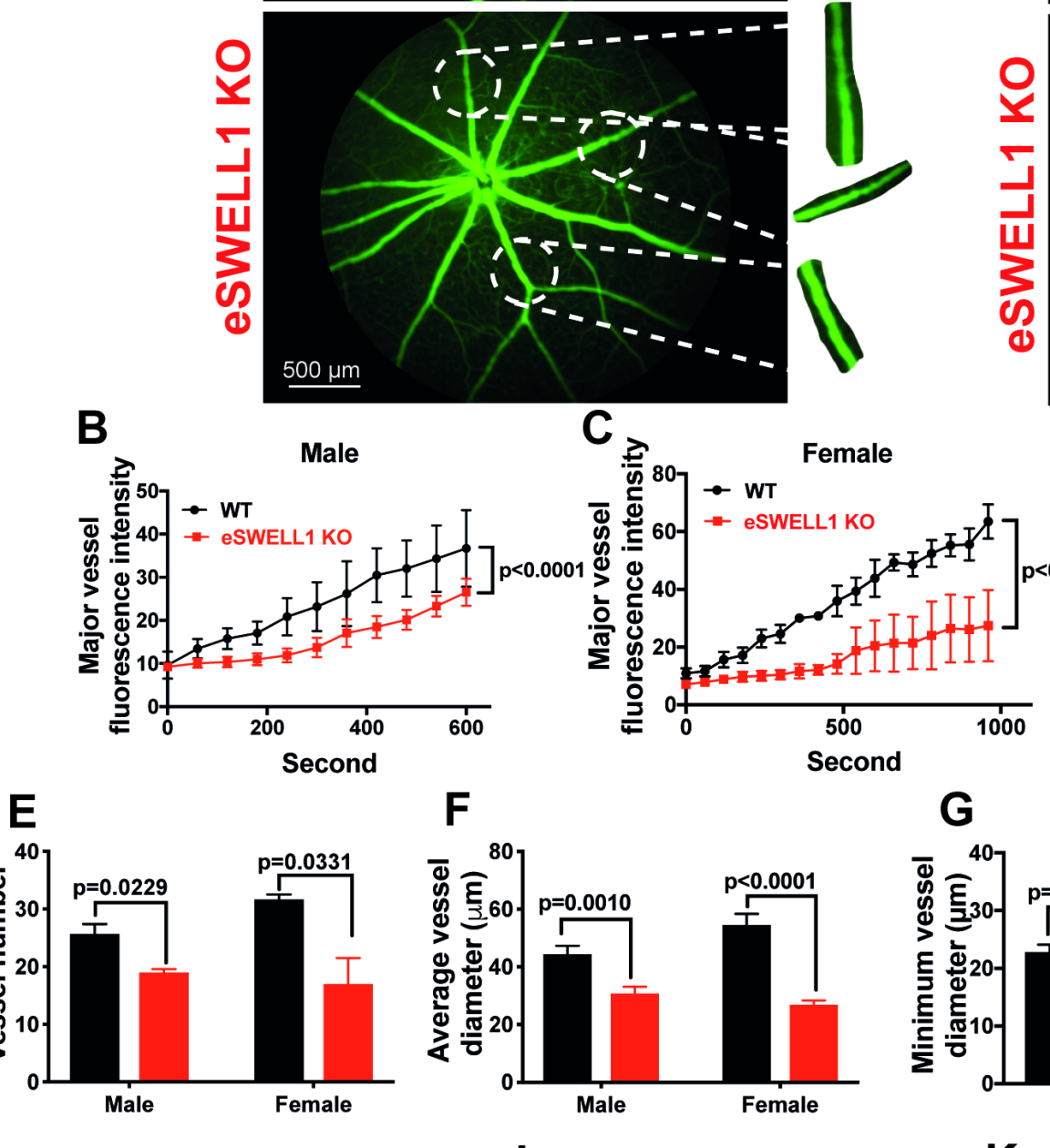

$\mathbf{F}$

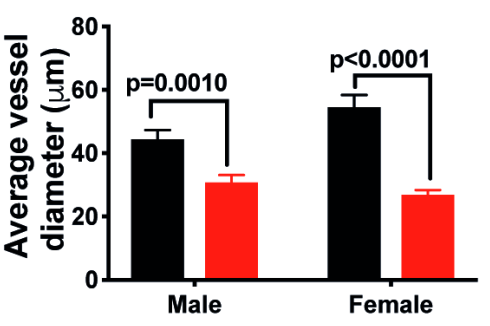

$\mathbf{G}$
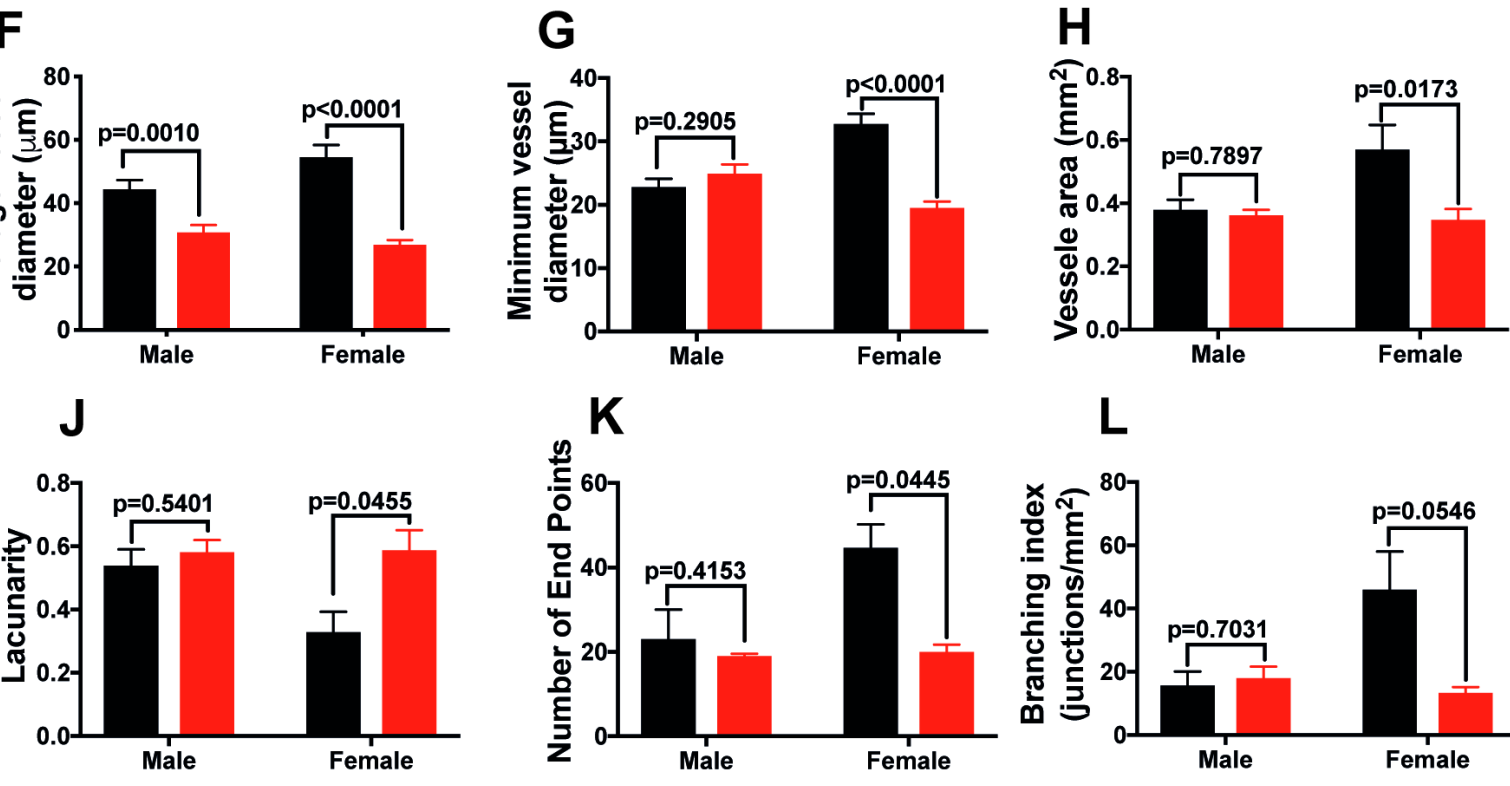

$\mathbf{L}$

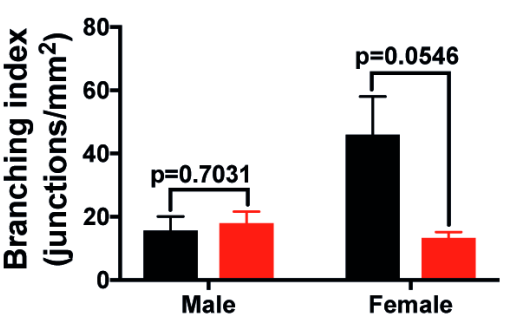

Figure 8. Endothelium-specific SWELL1 KO mice exhibit exacerbated impairments retinal microvascular disease in the setting of Type 2 diabetes. 

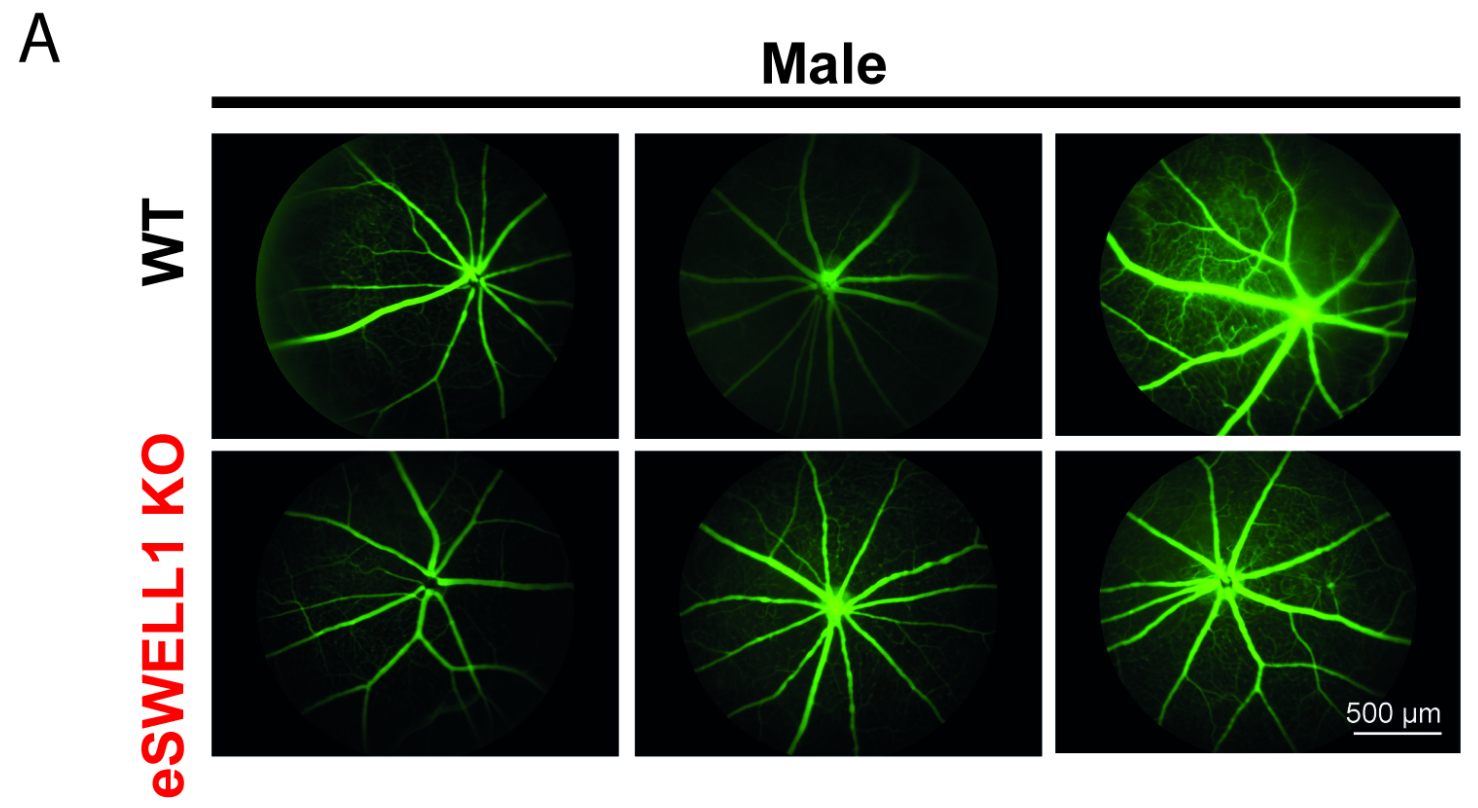

B

\section{Female}
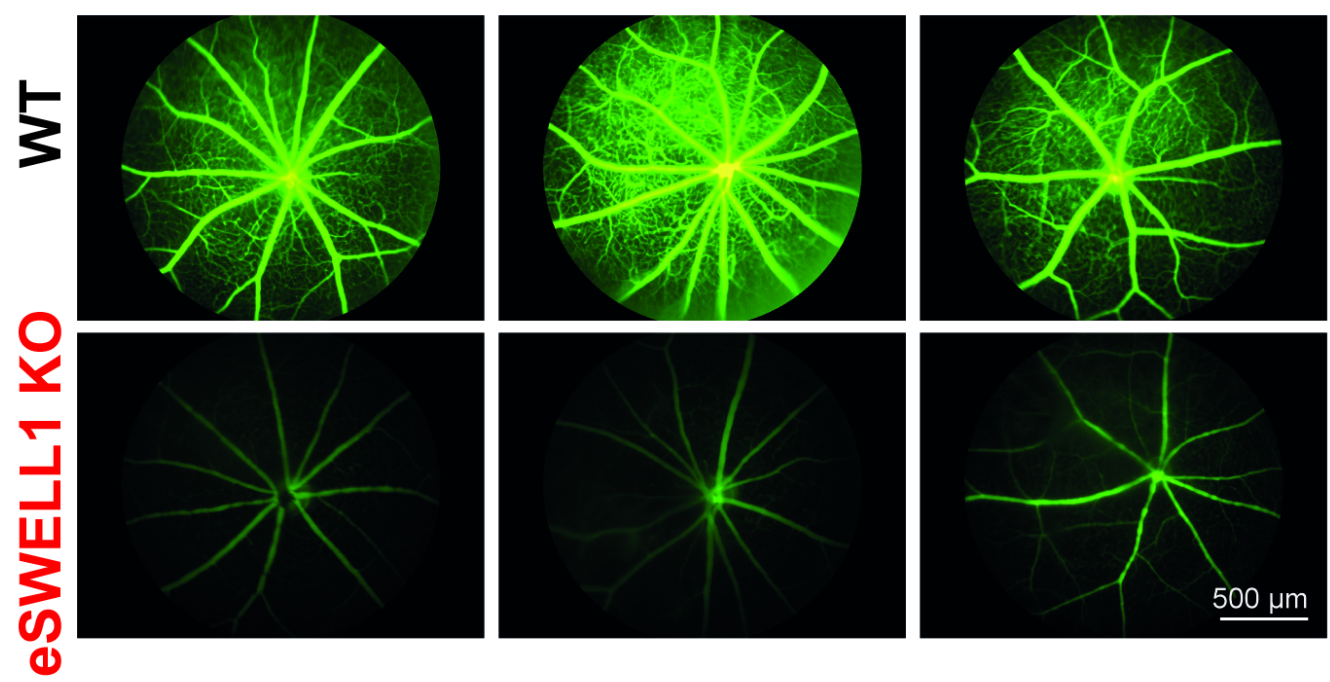

Figure 8- Figure Supplement-2. Endothelium-specific SWELL1 KO mice exhibit exacerbated impairments retinal microvascular disease in the setting of Type 2 diabetes. 
GTT

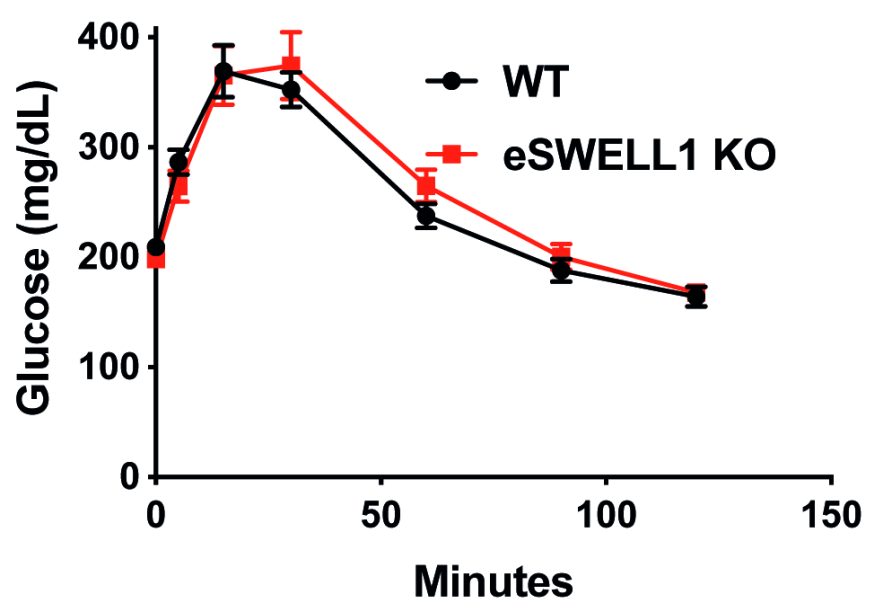

ITT

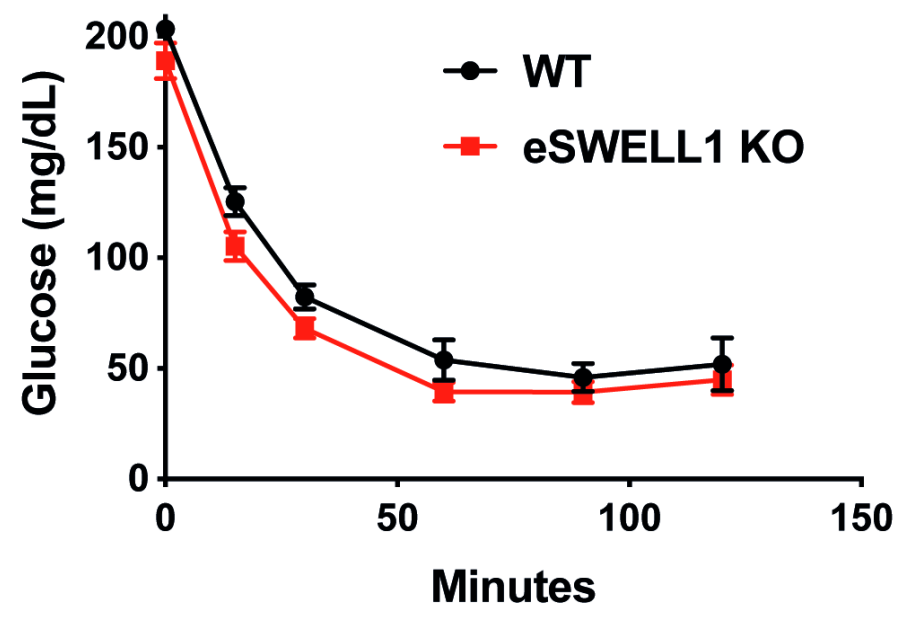

Figure 8- Figure Supplement-3. Glucose tolerance (GTT) and insulin tolerance (ITT) are not altered in endothelium-specific SWELL1 KO mice $(n=13)$ compared to WT mice $(n=8)$ raised on a high-fat high sucrose diet for 10 months. 